et à nouveau servent à de plus heureux constructeurs. II arrivera bien un jour où l'édifice sera stable et, ce jour-là, l'électrochimie deviendra notre plus active et notre plus riche industrie. Ne désespérons donc pas et tournons au contraire tous nos efforts de ce côté.$$
* *
$$

En voyant la diversité des moyens employés pour produire ces forces motrices colossales et la variété de leurs applications, on comprend mieux les difficultés qu'ont dû vaincre leurs créateurs, leur effort gigantesque et la grandeur de leur cuvre. Au contact de ces difficultés, par la connaissance approfondie decet effort, on acquiert une opinion plus nette, plus juste, sur la nature de l'appui qui doit être accordé à cette jeune et déjà si puissante industrie de la Houille blanche.

Sans doute, ces discussions savantes, ces études techniques, ces leçons de choses, n'ont pas donné sur l'heure un résultat tangible, mais elles ont semé des germes de progrès qu'un avenir prochain verra lever si l'Etat les dégage de to it: oppression.

De cette semence ainsi jetée dans le champ industriel de notre Pays, il faut remercier le Syndicat des Forces hydrauliques, l'habile initiateur du Congrès; les fruits qu'il portera c'est à lui que nous les devrons. Nos félicitations pour la parfaite exécution matérielle de tout ce qu'il avait si bien organisé, nous les lui adressons sincèrement; mais ce dont nous le louons par dessus tout c'est d'avoir provoqué ce grand mouvement d'opinion qui ouvre une ère nouvelle dans la marche en avant de notre industrie nationale.

E.-F. CôTe.

\section{COMPTE-RENDU DU CONGRËS}

\section{SECTION TECHNIQUE}

Président: M. Hart.', ingénieur, Président de la Société internationale des Electriciens, Président d'honneur du Congrès .

Vice-président: M. Cornuaurt, ingénieur, Président du Syndicat de l'Industrie du Gaz en France, vice-président du Congrès.

Secrétaire: E.-F. Côte, ingénieur à Lyon, rédacteur en chef de la Houille Blanche.

\section{Résumé des Conférences}

\section{ÉTUDE HYDROLOGIQUE D'UN BASSIN DE MONTAGNE}

(Résumé de la conférence faite au Congrès)

\author{
Par M. DE LA Brosse \\ Ingénieur en chef des Ponts et Chaussées
}

Lorsque l'on tente d'opérer un recensement des forces hydrauliques d'une région montagneuse, on s'aperçoit immédiatement que la détermination d'un des éléments dyna- miques des cours d'eau est entourée de grandes difficultés pratiques.

La puissance hydraulique est défnie par deux facteurs: la pente et le débit. Or, s'il est généralement facile d'obtenir la pente par des opérations simples ct rapides, ou même par un coup d'œil plus rapide encore sur une carte, il en est tout autrement du débit, et la première impression, dans les recherches de ce facteur, est plutôt décourageante. On ne posssède que fort peu de déterminations précises; dans la région des Alpes, la plupart des grandes installations hydroélectriques récentes se sont faites sur la foi de jaugeages summaires, très peu prolongés, souvent même restreints à une ou deux mesures de vitesses superficielles par flotteurs, aussi les écarts sont-ils grands entre les chiffres donnés, par divers intéressés, pour les débits d'étiage de certains grands cours d'eau, tels que le Drac.

Il serait évidemment très désirable d'organiser un système de mesures en vue de combler cette lacune, et cela ne paraît pas impossible si les divers intèressés veulent bien se prêter mutuellement le concours de leurs moyens d'action respectifs, comme nous le dirons plus loin.

Les méthodes de jaugeage les plus usuelles reposent sur l'emploi des déversoirs, des hydromètres (1) et des flotteurs de surface ou lestés.

Déversoirs. - Les déversoirs jouissènt, en général, d'une confiance justifiée, mais ils ne la méritent que lorsqu'ils reproduisent exactement les dispositions types des expériences qui ont servi à déterminer le coefficient empirique $\mathbf{m}$, qui figure dans la formule:

$$
\mathbf{q}=\mathbf{m} \omega \sqrt{\mathbf{2 g h}} \quad \text { (I) }
$$

qui donne le débit en fonction de la section $\boldsymbol{\omega}$ et de la charge $\mathbf{h}$ mesurée entre le seuil et le niveau de l'eau tranquille en amont de l'orifice.

l.es expériences classiques de Poncelet et Lesbros ont donné la valeur de $\mathbf{m}$ pour des déversoirs en mince paroi, longs de om 20 et de $o^{m} 60$ et pour des charges inférieuresà o $\mathbf{m}$. $3 \circ(\mathbf{m}=\mathbf{0}, \mathbf{3 7 5})$; celles de Boileau $(\mathbf{m}=\mathbf{0}, \mathbf{4 2 9})$ ont étendu notablement l'échelle des longueurs pour des déversoirs à contraction latérale nulle (jusqu'à $l=\mathbf{1 , 6 0}$ ). Hirn a opéré sur des seuils étendus de 3 et de 9 mètres $(\mathbf{m}=\mathbf{0}, \mathbf{4 3})$, Graeff sur un seuil de $1^{\mathrm{m}}$ bo au Furens et sur un autre de $10 \mathrm{~m} .(\mathbf{m}=\mathbf{0}, \mathbf{4 5})$ avec paroi épaisse $(0,15)$. Enfin M. Bazin a donné, à la suite d'expériences précises et trés prolongées, une sérịe complète de coefficients pour des seuils de 1,50 et de 2,00 , dans des conditions variées auxquelles on peut ramener la plupart des cas. Quelques auteurs (Boileau notamment) ont aussi donné des formules applicables aux barrages obliques ou en forme de chevrons qui ne sont guère usitées.

Malgré le nombre considérable de ces déterminations et peut-être mêmeà cause de leur multiplicité, l'on n'en est pas moins assez embarrassé toutes les fois qu'il s'agit de fairc

(1) Nous ne saurions énumérer, dans cet exposé sommaire, tous les procédés de jaugeage qui ont été appliqués ou indiqués par divers auteurs; il en est de fort ingénieux (mesures des températures, emplot de solutions salines titrées, etc., etc.), mais leur simple énumération nous entrainerait trop loin. 
un choix et de jauger par déversoir, d'autant plus qu'il faut des précautions minutieuses pour régler l'arête du seuil, ses distances au fond et aux parois latérales, sa parfaite horizontalité (I), la mesure précise de la charge, etc. C'est, sans doute, ce qui explique que l'emploi des déversoirs de jaugeage ne soit pas plus général.

Quoi qu'il en soit, et pour un premier aperçu approximatif, on emploie souvent une formule (dite formule française) très simple et qui supprime les tables de coefficients, c'est la suivante:

$$
\mathrm{q}=1,80 \mathrm{lh} V \overline{\mathrm{h}}
$$

dans laquelle $I$ représente la longueur du seuil et $h$ la charge entre ce seuil et le niveau des eaux tranquilles en amont. Cette formule dérive de la précédente $\left(\mathrm{n}^{\circ} \mathrm{l}\right)$ si l'on y donne à $\mathbf{m}$ la valeur fixe de $0,4^{2}$. Elle ne doit être regardée que comme un moyen grossier de calcul, mais elle peut rendre des services en donnant immédiatement un premier aperçu du débit.

Mesure de la pente. - Nous n'insisterons pas sur les jaugeages par mesure de la pente longitudinale au moyen des formules du mouvement uniforme dans les canaux découverts, nous dirons seulement qu'elles dérivent toutes de la formule type :

$$
\mathbf{R i}=\mathbf{a} \mathbf{u}+\mathbf{b} \mathbf{u}^{2}
$$

dans laquelle $\mathbf{R}$ représente le rayon moyen (ou rapport entre la section liquide et le périmètre mouillé), $\mathbf{i}$ la pente superficielle par unité de longueur, $\mathbf{u}$ la vitesse moyenne, $\mathbf{a}$ et $\mathbf{b}$ deux coefficients numériques plus ou moins compliqués et dont l'un peut être nul.

M. Bazin a donné, comme on le sait, la valeur des coeffcients correspondants à diverses natures de parois et ses formules sont souventemployées pour évaluer la capacité de débit de canaux de section connue. Il est plus rare qu'elles servent à des jaugeages proprement dits.

Hydromètres. - On appelle hydromètres les instruments qui servent à mesurer le débit par détermination de la vitesse. Les deux types principaux sont le moulinet de Voltmann et le tube piézométrique de Pilot-Darcy.

Moulinet de Voltmann. - Le moulinet est un instrument qui permet de mesurer la vitesse d'un courant liquide par la rotation qu'il imprime à des ailettes planes ou héliçoîdales montées obliquement sur un axe immergé dans le courant.

L'instrument doit nécessairement être taré, c'est-à-dire qu'il faut commencer par établir la relation qui, pour un instrument donné, existe entre la vitesse du courant et le déplacement angulaire de l'appareil mobile. Ce tarage ne va pas sans quelques difficultés pratiques, car il exige une organisation assez complète et un assez nombreux personnel. Le moulinet mis sous les yeux du Congrès a serviplusieurs années au jaugeage de l'Isère à Grenoble. Il porte un compteur de tours (àdeux roues) commandé par une vis sans fin placéesur l'axe de rotation de l'hélice. L'enclanchement des dents du compteur avec la vis sansfin s'opère sous l'action d'un double électro-aimant logé dans une boîte métallique portée par

(1) M. Fontès a montré l'importance des erreurs dues à une inclinaison du seuil et en a donné une évaluation précise. l'instrument. Un câble à trois fils isolés relie l'électro-aimant à une pile doñt les boutons permettent de mettre en prise le compteur avec sa vis ou de l'en séparer à volonté.

L'appareil porte une douille à pied qui glisse sur un tube de guidage en fer étiré,fendu le long d'une génératrice pour le passage du support de la douille, disposition réalisée pour la première fois par $M$. l'ingénieur Harlacher, sur le Danube.

Le tarage a été fait dans l'eau tranquille du fossé de la fortification, à Grenoble, pour des vitesses de déplacement variant de o à plus de 2 mètres ( 1 ). Il aurait été utile de refaire un nouveau tarage après chaque campagne, pour vérifier la fixité des coefficients, mais cela n'a pas eu lieu fautede temps.

L'emploi de ce moulinet a permis d'étudier la distribution des vitesses dans une section transversale de l'Isère pour diverses hauteurs d'eau; il serait trop long d'entrer ici dans le détail des expériences, nous nous contenterons done d'indiquer que cette distribution n'est point celle que l'on s'attend parfois à trouver: la vitesse maximum n'a pas lieu à la surface, ni surl'ordonnée de plus grande profondeur, elle est réalisée à une profondeur variable avec l'état du courant et la direction du vent (à o m. 50 ou i m. oo environ) et se trouve notablement en dehors de la ligne du thalweg.

La vitesse moyenne est d'environ $67 \%$ de la vitesse maximum superficielle.

Tube piézométrique. - Le tube piézométrique est un instrument qui donne immédiatement la vitesse d'un filet liquide par la hauteur de la charge qui lui correspond. L'appareil, mis sous les yeux du Congrès, se compose de deux tubes en verre, réunis à leur partie supérieure, d'oú part un tube de caoutchouc qui permet d'y faire, par aspiration, un vide partiel. Les tubes sont recourbés à leur partie inférieure et percés chacun d'un petit orifice dont l'un est directement opposé au courant et reçoit, par conséquent, la pression correspondant à la vitesse, et dont l'autre, ouvert sur le côté, à l'abri d'une gaine cylindrique enveloppante, donne la charge hydrostatique. La différence des niveaux de l'eau dans les deux branches donne la hauteur due à la vitesse qui peut se lire immédiatement sur une échelle graduée où circule un index mobile muni d'un vernier. Cet instrument est d'un usage rapide et il donne des indications suffisamment précises dès que la vitesse estun peu forte, mais, dans les faibles courants; son emploi n'est guère pratique.

Flotteurs. - Le jaugeage par flotteurs est trop connu pour qu'il y ait à le décrire ici; nous dirons seulement qu'il peut être utilement perfectionné par l'emploi de flotteurs lestés qui donnent la vitesse moyenne sur l'ordonnée de leur parcours, lorsqu'ils possèdent une plongée des $5 / 6$ de la profondeur. D'ailleurs, il est utile, quand on le peut, de contrôler les jaugeages ainsi obtenus par l'emploi d'un moulinet ou d'un tube de Darcy et il est toujours nécessaire de bien préciser les conditions de l'opération, l'itinéraire de chaque flotteur, les anomalies constatées et les détails du calcul.

(I) On a fait 27 mesures qui ont permis d'établir les coefficients de tarage de linstrument par la méthode des moindres carrés. Le détail des calculs en sera donné dans le compte-renda des séanc:s du Congrès. 
Nous nous en tiendrons à ces indications sommaires sur les procédés de jaugeages, en exprimant le vœu que les industriels et les divers services locaux intéressés veuillent bien organiser sur les principaux cours d'eau de la région des Alpes des jaugeages méthodiques en employant diverses méthodes pour contrôler les résultats de celles qui leur sont le plus facilement applicables. Il importe absolument, dans tous les cas, d'établir sur un certain ' nombre (1) de jaugeages précis, des formules de débit qui permettent d'obtenir, chaque jour, le débit de la rivière par une simple lecture de hauteur à l'échelle voisine (2).

Ces hauteurs, transformées aussitôt en débits, servent à construire par points le graphique des débits de la station et fournissent, pour l'étude des cours d'eau, les données extrêmement précieuses qui font aujourd'hui presque totalement défaut.

Importance des graphiques des débits. - Cependant, et pour finir sur des perspectives plus consolantes, nous dirons que l'on possède, pour quelques points privilégiés, des indications de débit sérieuses. La Société de la Volta, en amont de Moûtiers, a recueilli, pendant plusieurs années, des données assez complètes sur l'Isère et l'on peut en tirer déjà, tout au moins, un aperçu des variations de son régine.

Il ne sera pas sans intérêt de mettre sous les yeux du Congrès un graphique des débits d'un petit cours d'eau de montagne, le haut Bréda, obtenus par MM. Bergès à la suite de jaugeages par déversoirs poursuivis pendant trois années consécutives.

Ce graphique montre la grande amplitude des variations pour un même mois et fait ressortir l'importance d'observations quotidiennes patiemment poursuivies pour la connaissance sérieuse du régime d'un cours d'eau. En cette matière les moyennes ne suffisent point, ce sont les valeurs journalières qu'il faut connâtre si l'on veut savoir à quoi s'en tenir sur les débits et si l'on ne veut pas s'exposer à de graves mécomptes.

Dans le cas spécial du haut Bréda, il s'agit d'une chute d'environ 1100 mètres réalisable entre le plateau des Sept-Laux (altitude 2150), et le fond de France (altitude ro50) sur un modeste ruisseau dont le débit est descendu, en février 1893 , à un minimum extrême de 2r litres par seconde et dont les crues peuvent dépasser booo litres (3). Ces énormes variations ne sont pas, d'ailleurs, sans intérêt, car le cours d'eau issu des lacs où il se clarifie peut être facilement régularisé et son débit minimum porté à environ 500 ou 600 litres par un emmagasinement convenable des volumes surabondants dans la prícieuse réserve des lacs supérieurs. C'est là un exemple bien intéressant de ce que peut donner un aménagement des

(1) Un petit nombre de jaugeages suffit pattaitement pour arrêter une formule de débit, pourvu que le lit soit fixe et le courant régulier.

(2) C'est ce qui existe déjả dans les services de navigation des fleuves principaux et c'est ce qui fonctionne en Suisse d'une façon assez étendue par les limnimètres er limnigraphes eriregistreurs installés dans les divers bassins.

(3) Le bassin alimentaire possécie une supericite de 1380 hegtates. lacs, même les plus petits (r), quand ils sont abondamment alimentés. MM. Bergès, qui ont sut tirer un si remarquable parti du lac Crozer pour la régulation du ruisseau de Lancey, réussiront aussi, ce n'est pas donteux, l'aménagement des lacs des Sept-Laux, au grand profit de l'industrie, dans un rayon très étendu.

Généralisation des résultats. - Quel que soit, cependant, l'intérêt qui s'attache à l'organisation générale méthodique des jaugeages si rares jusqu'à ce jour, ou ne peut se flatter de les multiplier assez pour arriver ainsi à la connaissance directe du régime de tous les cours d'eau utilisables des Alpes. Il faudra toujours étendre à un certain nombre de bassins (sauf à les corriger comme il conviendra) les résultats acquis pour le petit nombre relatif de ceux où l'on peut organiser des mesures suivies quotidiennes. Or, c'est là une nouvelle branche d'études qui mérite également l'attention.

Elle fait, depuis plusieurs années, en Suisse, l'objet d'un travail considérable dont une partie est déjà publiée et qu'il sera intéressant de résumer, tout au moins, en quelques mots.

Chaque bassin est mesuré sur la carte, puis décomposé d'abord en zones d'altitude de 300 en 300 mètres, ensuite en natures de terrains (rochers et éboulis, forêts, névés et glaciers, lacs, autres terrains) A chacune de ces catégories seront appliqués des coefficients d'alimentation pluviale, d'évaporation, d'absorption et de ruisselement déduits des données météorologiques et géologiques connues, qui permettront sans doute de définir complètement et avec une grande exactitude le régime hydrologique de chaque bassin.

Un pareil travail serait certainement très utile pour les Alpes françaises et les ingénieurs spécialement chargés de missions d'étude sur les forces hydrauliques se proposent de le poursuivre sur des bases à peu près semblables. Toutefois leur mission serait grandement facilitée et porterait certainement beaucoup plus de fruits si les industriels voulaient bien leur prêter leur précieux concours pour la recherche des données relatives aux bassins dont ils utilisent les eaux.

L'auteur de ces lignes aime à espérer que ce concours ne fera pas défaut et que le Syndicat des industriels qui a si bien organisé le Congrès voudra bien centraliser les résultats obtenus par ses adhérents et en faire profiter tous ceux que ces études intéressent.

A cet effet, il convient que les industriels veuillent bien suivre un programme identique et adopter des notations uniformes qui facilitent les comparaisons.

Il sera très utile aussi qu'ils fassent connaître leur avis sur certaines questions qui pourront leur être posées et dont une peut être indiquée immédiatement dans les termes que voici : (2)

(1) La surface du plus grand lac des Sept-Laux, le lac Cotepen, ne dépasse pas 30 hectares.

(2) Les principaux ètats susceptibles d'une représentation graphique sont: $1^{\circ}$ Le minimum minimorum (extrêtne étiage exceptionnellement réalisé de loin en loin et toujours très court! partois un jour tous les 5 ou 6 ans) ; $2^{\circ}$ La moyenne du mois le plus faible; 30 La moyenne des trois mois les plus faibles ; $4^{\circ}$ La moyenne des 6 mois les plus faibles; 50 Le minimum des 9 mois les plus forts 
Quel serait, de l'avis de chacun, l'état caractéristique d'un cours d'eau le plus utile à représenter graphiquement sur une carte, pour donner, à première vue, une idée de sa puissance hydraulique?

Un tableau, mis sous les yeux du Congrès et dont l'en-tête est ci-après, réunit ces diverses données.
Quant à l'étude hydrologique des divers bassins quelques simplifications semblent pouvoir être apportées au type suivi en Suisse : on peut fort bien adopter des divisions de 500 ou 500 mètres (au lieu de 300) pour les zones d'altitude. De même, il ne parât pas utile de faire une catégorie à part des lacs dont l'étendue relatice est généralement

TABIEAU I

\begin{tabular}{|c|c|c|c|c|c|c|c|c|c|c|c|c|c|c|c|c|}
\hline \multirow[b]{3}{*}{ 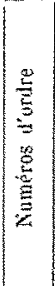 } & \multirow[b]{3}{*}{$\begin{array}{l}\text { nésignation } \\
\text { des sechrns de } \\
\text { cours d'ealu }\end{array}$} & \multirow{3}{*}{ 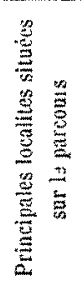 } & \multirow{3}{*}{ 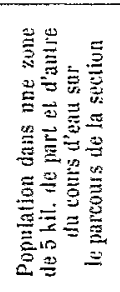 } & \multirow{3}{*}{ 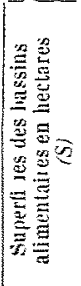 } & \multirow{3}{*}{ 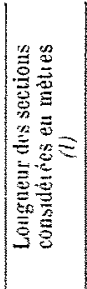 } & \multirow{3}{*}{ 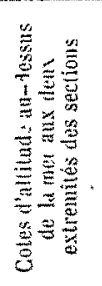 } & \multirow{2}{*}{\multicolumn{2}{|c|}{ ['ente de lit sectron] }} & \multicolumn{7}{|c|}{ 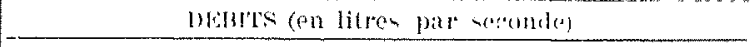 } & \multirow{4}{*}{ 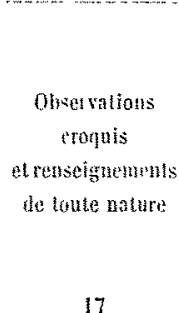 } \\
\hline & & & & & & & & & \multicolumn{6}{|c|}{1 Porime de lat sentron } & \multirow[b]{2}{*}{ 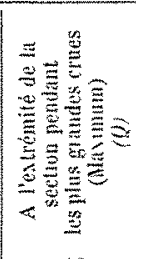 } & \\
\hline & & & & & & & 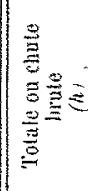 & 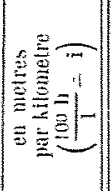 & 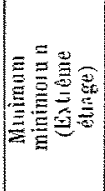 & 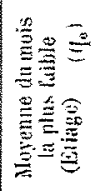 & 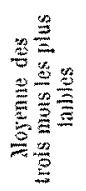 & 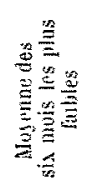 & 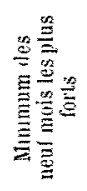 & 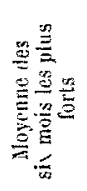 & & \\
\hline 1 & 2 & 3 & 4 & 5 & 6 & 7 & 8 & $\theta$ & 10 & 11 & 19 & 13 & 11 & 15 & 16 & \\
\hline
\end{tabular}

IAPIEAU II

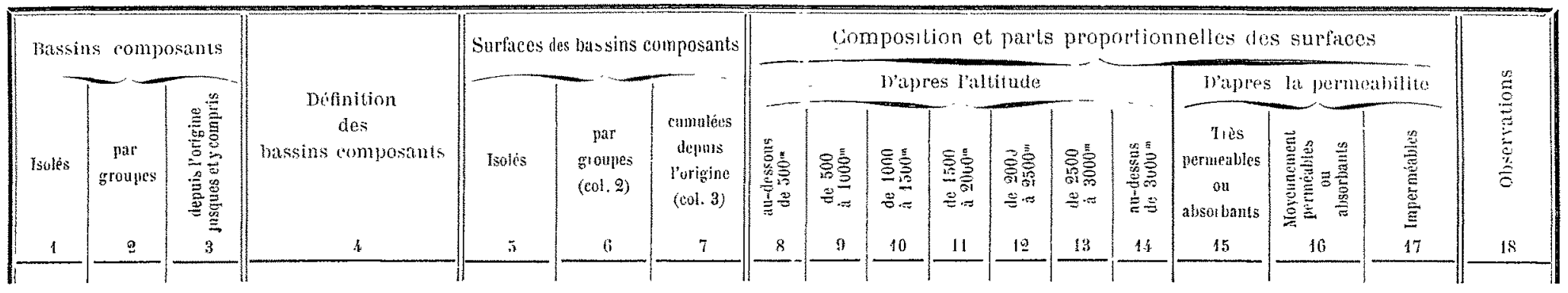

minime dans les bassins alpestres français ; les rochers, au contraire, ont des pouvoirs absorbants fort divers, suivant leurs natures; certaines roches calcaires (1) des massifs subalpins sont éninemment perméables par leurs innombrables fissures, tandis que les roches cristallines anciennes des massifs alpins sont presque complètement imperméables. Il serait donc possible, et sans doute avantageux au point de vue de la rapidité d'exécution du travail, d'adopter une division des terrains beaucoup plus simple et ne contenant, par exemple, que les trois classes indiquées sur l'entête du tableau II ci.dessus, également mis sous les yeux du Congrès.

Avant de rien arrêter de défnitif sur ces divers détails, il est utile de connaître l'avis de chacun et c'est à le faire con. naître que le soussigné croit devoir, en terminant, convier les intéressés. - R. DE LA Brosse.

\section{$*^{*} *$}

A propos de la mesure du débit des cours d'eau, M. Boucherot. docteur ès-sciences, ingénieur-èlectricien, à Paris, soumet au Congrès, lidée suivante :

Essayer d'appliquer la méthode imaginée par M. Boury, professeur de physique à la Sorbonne, pour déterminer la vitesse d'une veine liquide en fonction de la différence de potentiel que l'on crée entre deux points de cette veine, lorsqu'on la soumet à l'influence d'un champ magnétique.

Considérons un cours d'eau dont la direction est perpendiculaire, ou même inclinée, sur celle du champ. magnétique terrestre; plaçons verticalement, dans ce cours d'eau,

(1) Cela est particulièrement sensible pour les calcaires urgoniens et sénoniens, très développés dans les régions des ahaines subalpines dauphinoises et provençales. deux tiges métalliques, sur une ligne normale à la direction du courant, c'est-à-dire dans le sens du champ magnétique, et relions-les, d'autre part, à un appareil de mesure très sensible. Nous avons ainsi constitué un circuit fermé dont un élément, la lame liquide comprise éntre les deux tiges métalliques, "est mobile normalemennt aux lignes de forces du champ terrestre; il naît, dans ce circuit, une force électromotrice dont la valeur est fonction de la rapidité de déplacement de la veine liquide.

Cette méthode permet d'obtenir, en laboratoire, des indications précises. M. Boucherot suppose qu'en l'appropriant à la mesure du débit des cours d'eau, l'on pourrait peut-être en obtenir des résultats intéressants. Mais il fait bien remarquer que c'est seulement une idée qu'il soumet aux hydrauliciens et il leur laisse le soin d'en juger la valeur au point de vue pratique.

\section{INFLUENCE DES NEIGES ET DES GLACIERS SUR LE RÉGIME DES COURS D'EAU}

\section{Détermination des aébits caractéristiques de la valeur industrielle d'une chute d'eau.}

\author{
Confirence de $M$. René Tavernier \\ Ingenieur en Chef des Ponts-et-Chausscés.
}

Exposé. - M. R. Tavernier se propose, par quelques commentaires très brefs des deux graphiques qu'il met sous les yeux du Congrès, de chercher à caractériser l'influence très variable, suivant les bassins et suivant laltitude, des glaciers et des neiges sur le régime des cours 
d'eau qu'ils alimentent. Il se propose en même temps d'examiner quels sont les débits qui peuvent le mieux servir à déterminer la valeur industrielle des forces hydrauliques alpestres. Pour cela, il va comparer le régime de la Durance, près d'Avignon, dans une région où les pluies jouent leur rôle à côté des neiges, avec le régime du Haut-Rhône à Saint-Maurice, cours d'eau qui est au contraire affecté d'une façon prédominante par la « Houille blanche ».

Pour que la comparaison fut tout à fait instructive et complète, il serait nécessaire de pouvoir mettre en parallèle surl'un et sur l'autre cours d'eau des points de même altitude, par exemple Sisteron sur la Durance et SaintMaurice sur le Rhône; puis de choisir successivement dans un même bassin les points d'altitudes différentes, par exemple sur la Durance, Briançon, Sisteron, Avignon. On marquerait par des trais généraux, d'abord l'influence de la "Houille blanche " dans chaque bassin et ensuite l'atténuation progressive de cette influence à mesure qu'en s'abaissant on s'éloigne des sommets.

Mais M. Tavernier n'ayant pas sous la main les éléments d'une étude aussi complète se borne à analyser leș conséquences qui se peuvent déduire des deux graphiques en question qui ont été empruntés, le premier (no $\mathrm{I}$ ), aux travaux de M. Imbeaux sur la Durance, l'autre ( $n^{0} 2$ ) aux recherches que $M$. Van Muyden a été appelé à faire,comme expert de la ville de Lausanne, en vue de son installation hydro-électrique.

Les graphiques représentent le débit moren mensuel de chacun des douze mois. La courbe du milieu en trait fort correspond à la moyenne des débits moyens mensuels reldtive à un certain nombre d'années (dix ans pour le Rhône, sept ans pour la Durance). Les courbes en traits fins correspondent, l'une à l'année la plus sèche et l'autre à l'année la plus humide de la périude considérée.

\section{Régime du Haut-Rhône à Saint-Maurice.}

M. Van Muyden, en prenant la moyenne des dix années qu'il considere, indique les nombres de jours par an pendant lesquels le débit du Haut-Rhône varie entre $2 \mathrm{I}$ et $3 \mathrm{o}^{\mathrm{m}} \mathrm{m}^{3}$ (19 jours), entre 30 et $4 \mathrm{O}^{\mathrm{m}^{3}}$ ( 39 jours), etc. Ces données permettent de construire la courbe de régime du HautRhône (diagramme No III) et de déterminer graphiquement à l'aide de cette courbe, les deux débits caractéristiques suivants, que M. Tavernier considère comme étant les plus utiles à connaître au point de vue industriel. On trouve ainsi que le débit caractéristique d'étiage, c'est-à-dire le débil au dessous duquel le Haut-Rhône descend, année movenne, pendant dix jours par an au plus, est de $25 \mathrm{~m}^{3}$ et que le débit caractéristique moyen, c'est-à-dire le débit an dessous duquel le Haut-Rhône descend, année morenne, pendant six mois par an au plus est de $72^{\mathrm{m} 3}$. Les résultats seraient fort différents si, au lieu de l'année moyenne, on considérait une année sèche; par exemple, en 1894, les débits sont restés pendant six mois inférieurs non à $72^{m^{3}}$ mais à $44^{\mathrm{m}^{3}}$ seulement, alors qu'au contraire le débit caractéristique moren de 1897 (année de crues) s'est élevé à $93^{3 n^{3}}$.

Si au lieu de considérer la courbe de régime qui permet de déterminer exactement les débits caractéristiques et les puissances correspondantes, on envisageait la courbe (gra- phique No II) des débits moyens mensuels, on pourrait retrouver, avec une grande approximation, les deux débits caractéristiques; le débil mensuel minimum (janvier et février I 894) aurait à peu près la même valeur $\left(25 \mathrm{~m}^{3}\right.$ et $\left.24^{\mathrm{m}^{3}}\right)$ que le débit caractéristique d'éliage défini par la condition que les débits faibles ne durent pas plus de dix jours par an.

Pour avoir le débit caractéristique moren il suffira de déterminer, par ses deux extrémités situées sur la courbe de l'année moyenne, une abcisse longue de six mois; cette abcisse allant dans le cas actuel, du 26 avril au 26 octobre, correspond à un débit de $72^{\mathrm{m}^{3}}$.

Cette seconde détermination des débits caractéristiques, moins rigoureuse que la première, a cet avantage de fournir certaines indications que la première ne donne pas. Elle montre qu'en général les débits les plus bas du HautRhône se produisent en janvier et en février, plus rarement en novembre et en décembre. Elle montre aussi qu'en général les débits correspondant à la puissance maximum seront atteints d'une façon continue de mai à octobre. Sans doute, il y aura des années sèches où la puissance moyenne sera réduite, des années humides où elle sera accrue, des chevauchements (probablement assez peu prononcés en ce qui concerne le Haut-Rhône) de la période des basses eaux sur celle des hautes eaux, ou inversement; et la considération de la courbe de débits journaliers permettra de se rendre compte de l'importance des écarts, suivant les années, entre les chiffres réels et les chiffres moyena. Mais il n'en reste pas moins évident que c'est sur ces derniers seuls que l'on peut asseoir les bases d'une statistique de l'ensemble de la puissance industrielle des cours d'eau.

Régime de la Durance à Bompas. - Si maintenant nous considérons la Durance à Bompas, près d'A vignon, ce qui frappe tout d'abord quand on compare les trois courbes du graphique No I à celles du graphique No II, c'est l'irrégularité de l'allure de chacune des courbes correspondant aux années sèche et humide par rapport à celle de l'année moyenne. Pendant une seule période, celle de la fonte des neiges et des glaciers, qui dure de juin à août, se terminant, par suite de l'insuffisance des neiges approvisionnées par le massif du Pelvoux, un mois au moins plus tôt que dans le Haut-Rhône, on peut observer entre les trois courbes une certaine similitude d'allure. Mais le printemps et l'automne, secs ou humides suivant les années, correspondent soit à de hautes eaux, soit à de basses caux. En général, comme le fait observer M. Inbeaux, la Durance traverse deux périodes de hautes eaux en juin et en novembre et deux périodes d'étiage en août et janvier. Mais en réalité les hautes eaux de juin et les basses eaux de la fin d'août présentent seules une allure un peu régulière, et cette régularité tient à la fonte des neiges.

M. Imbeaux estime que la neige tombée dans le bassin de la Durance représente, en moyenne, $39 \%$ de la pluie totale et correspond à I $8 \mathrm{r} 4$ millions de mètres cubes. Si l'on rapproche ce chiffre de la capacité, pourtant énorme, du réservoir de Serre-Ponson (200 millions de mètres cubes) projeté par les ingénieurs des Hautes-Alpes pour atténuer les pénuries de la Durance, on constate combien la régularisation des cours d'eau par les neiges, même quand son 
efficacité est limitée, comme cela arrive pour la Durance, et qu'elle ne s'exerce pas jusqu'au bout de la saison sèche, s'applique à des masses plus consicérables que la régularisation par des réservoirs artificiels. Mais er même temps il ne faut pas perdre de vue que le rôle capital des réservoirs artificiels sera précisément de corriger les anomalies les plus accusées des phénomènes naturels sur lesquels l'homme n'a pas d'action.

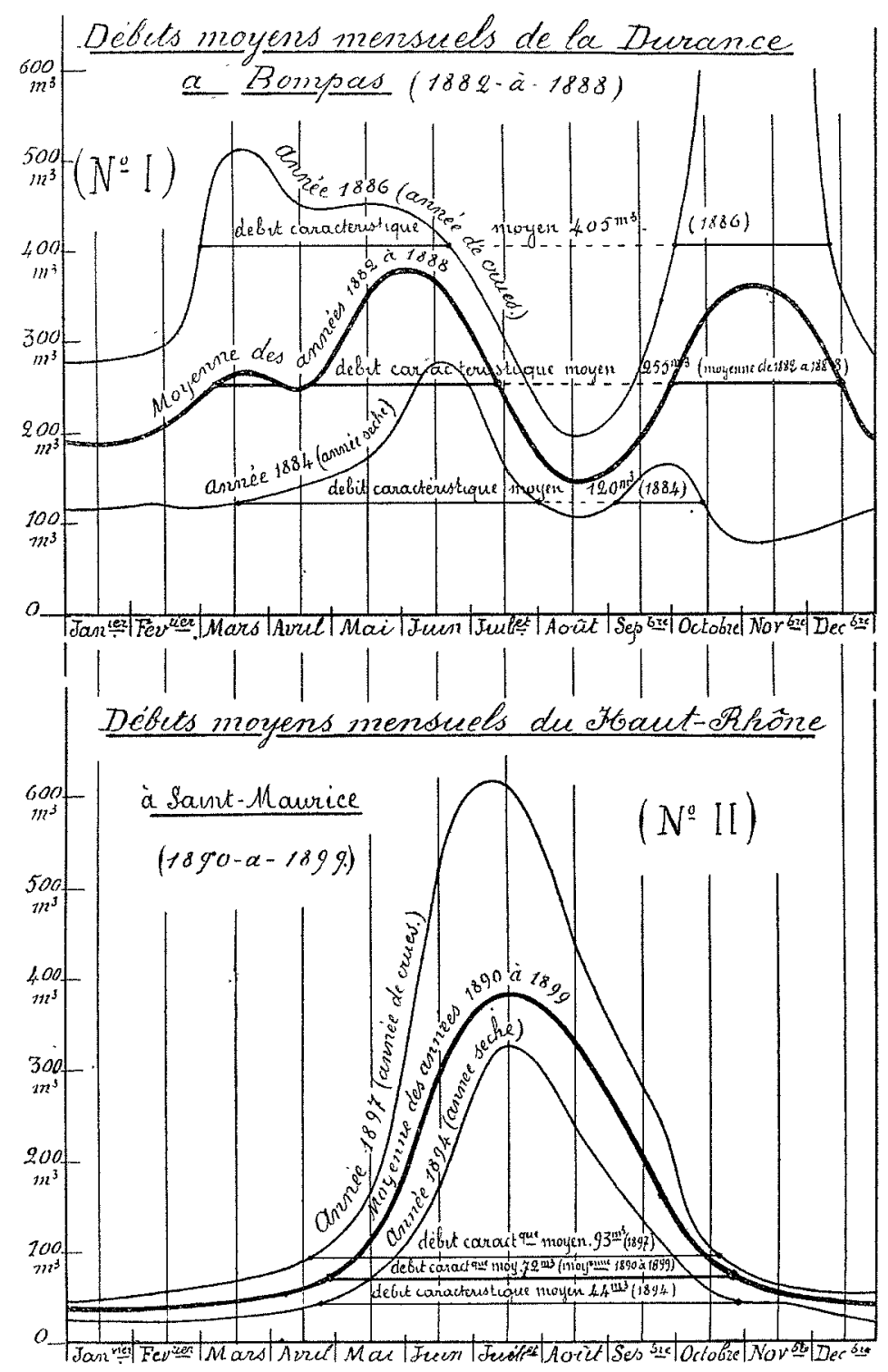

Si l'on voulait déterminer pour la Durance, comme cela vient d'être fait pour le Haut-Rhône, les deux débits caractéristiques dont on a lu plus haut la définition, on se trouverait, par suite de l'écart entre les trois courbes des débits mensuels, en présence de chiffres extrêmement différents, suivant que l'on considérerait, soil une moyennne de plusieurs années, soit une année extrême, sèche ou humide. Si l'on considère la moyenne de 7 ans, allant de 1882 à 1888, le débit caractéristique d'étiage devrait être fixé (novembre 1884 ) à.................... I $84^{\mathrm{m}^{3}}$ et le débit caractéristique moren, à.......... $255 \mathrm{~m}^{3}$ La période de six mois, pendant laquelle le débit moyen est atteint ou dépassé, se trouvera partagée, entre les deux saisons du printemps et de l'automne, par fractions à peu près égales.

Si l'on prend l'année sèche 1884 , le débit caractéristique moyen sera seulement de $120^{\mathrm{m}^{3}}$.
Si l'on envisage, au contraire, l'année de crues i 886 , le débit caractéristique moyen $405^{13^{3}}$ dépassera de plus du triple celui de l'année sèche.

Il laut remarquer qu'il s'agit là d'irrégularités qui, échappant à toute prévision, sont particulièrement gènantes au point de vue industriel.

On comprend bien, par exemple, qu'une industrie électro-chimique ou électro-métallurgique puisse s'accommoder d'intermittences très accentuées quand la périodicité annuelle en est à peu près connue d'avance, comme c'est le cas du Haut-Rhône et des torrents franchement glaciaires. Mais, quand il s'agit de variations qui dépassent la longueur d'une année et ne peuvent plus être prévues, il en est tout autrement. Supposons que laDurance (I), à Bompas, ait été aménagée en vue du débit caractéristique moyen de $255^{\mathrm{m}^{3}}$ correspondant à la moyenne 1882-1888. Quelles sont les industries qui pourront supporter que ce débit de $255 \mathrm{~m}^{3}$ n'ait été atteint en 1884 que pendant une partie du mois de juin, alors qu'au contraire, en ı 886 , il a été dépassé pendant l'année toute entière à l'exception du mois d'août?

Une seule catégorie d'entreprise, peut-être, pourrait trouver quelque intérêt à aménager et à utiliser ce débit si aléatoire de $255 \mathrm{~m}^{3}$. Ce serait une vaste entreprise de distribution régionale d'énergie, groupant solidairement les forces hydrauliques de plusieurs cours d'eau soumis à des influences pluviométriques différentes et complétée en même temps par d'autres sources d'énergie, telles que des machines à vapeur fonctionnant quelques mois par an. Des entreprises de cette envergure, munies de fortes réserves, pourront très bien supporter de brûler plus de charbon une année et moins une autre.

Conclusions. - "On ne peut avoir la prétention, dit M. R. Tavernier, en faisant l'inventaire de notre richesse hydraulique, de prévoir tous les cas si divers que pourra présenter son utilisation.

"Il est utile, assurément, pour que les chiffres soient comparables, de dresser partout cet inventaire sur les mêmes bases. Mais il est indispensable, en même temps, de bien marquer les circonstances, particulières au régime de chaque cours d'eau, qui pourront influer sur la paleur industrielle de forces hydrauliques mesurées partout à l'aide de ces bases.

« Dans cet ordre d'idées, nous proposerons, comme conclusion pratique de cette étude, d'utiliser, pour calculer la puissance industrielle d'un cours d'eau, les deux débits caractéristiques définis comme il suit :

" $1^{\circ}$ Le débit caractéristique d'étiage au-dessous duquel le cours d'eau descend pendant $\mathbf{1 0}$ jours par an au plus.

" $2^{\circ}$ Le débit caractéristique moyen, au dessous duquel le cours d'eau descend pendan 180 jours an plus.

"Chacun de ces débits pouvant ĉtre calculé à l'aide d'observations portant sur une ou plusieurs années, il importera de préciser la valeur des éléments qui ont servi aux calculs en faisant suivre leur définition, telle qu'elle est libellée ci-dessus, de la parenthèse (moyenne des années à ) ou simplement (année ).

(1) Hypothèse d'ailleurs irréalisable, puisque les eaux de la BasseDurance sont absorbées par les irrigations. 
" Le premier débit, l'étiage indnstriel, servira à calculer la puissance minimum sur laquelle on peut compter pendant tonte l'année, défalcalion faite de dix jours de chômage ou de travail réduit.

« Le second débit servira à calculer la puissance sur iaquelle on peut compter, année moyenne, pendant six mois par an.

"Des explications contenues dans cette note il résulte que l'on obtiendra, par ce second calcul, la puissance-limite que des industriels, dans le cas d'un cours d'eau fortement régularisé par la fonte des glaciers ou des neiges, peuvent être conduits à aménager.

" Dans le cas d'un cours d'eau principalement soumis aux intermittences des pluies, les industriels n'auront pas intérêt, en général, à pousser l'aménagement jusqu'à cette puissance-limite (1).

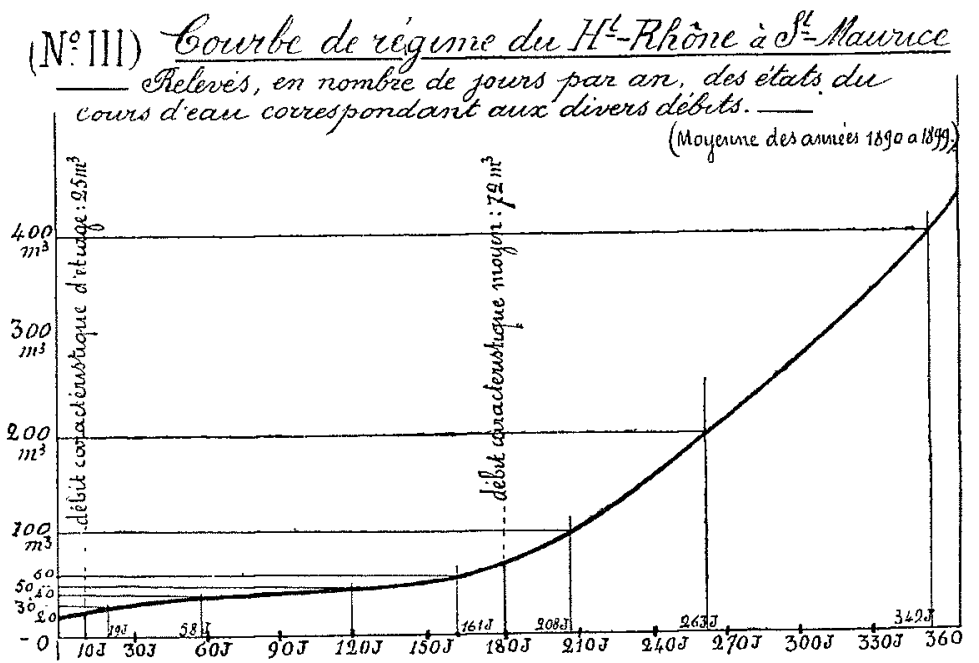

NOTA. - Le graphique donne le nombre de jours par an pendant lesquels le débit est égal ou inférieur à une valeur donnée.

Exemples: Le débit reste pendant 19 Jours par an, égal ou inférieur à $3 \mathrm{om}^{3}$; pendant 58 Jours égal ou inférieur à $40^{\mathrm{m}^{3}}$ ou, ce qui revient au même, pendant 39 jours, compris entre $30^{m^{3}}$ et $40^{m}{ }^{3}$. D'après ce graphique les deux débirs caractéristiques correspondant aux deux périodes de 10 Jous et de 180 Jaurs sont de $25 m^{3}$ et $7^{2} \mathrm{~m}^{3}$.

"Pour arriver le plus promptement possible à une connaissance, au moins approchée, des deux débits caractéristiques, on devra organiser, en utilisant tout d'abord et complétant, dans la mesure nécessaire, les lectures d'échelles faites par les Services d'annonce de crues, un réseau complet et méthodique d'observations hydrométriques.

"Si l'on dispose de jaugeages permettant de transformer les hauteurs d'eau en débits, on construira de suite la courbe des débits journaliers, ainsi que la courbe de régime, et on en déduira par les constructions graphiques, indiquées dans cette note, les deux débits caractéristiques.

“ Si le cours d'eau n'est pas jaugé ex qu'on dispose seulementde relevés hydrométriques relatifs à une série d'arnées, on pourra directement, d'après ces relevés, déterminer les deux hauteurs d'eau correspondant aux deux débits caractéristiques et se contenter d'effectuer des jaugeages aux environs de ces deux hauteurs.

(r) Ces conclusions ne s'appliquent qu'aux cours d'eau à écoulement libre et non à ceux susceptibles d'ètre régularisés par des réservoirs.
(En l'absence de tout jaugeage, l'examen des graphiques de hauteurs d'eau permettra d'apprécier les conditions de régularité des débits caractéristiques, ainsi que les époques où ils sont en général atteints. »

\section{CONFÉRENCE}

\section{SUR LA RÉGULARISATION DU DEEBIT DES COURS D'EAU AU MOYEN DES LACS OU DES RÉSERVOIRS ARTIFICIELS}

\author{
Par M. Crolaril
}

Ingénieur, directeur de la Papeterie de Cran, près Annecy.

Vous ne donnons qu'un résumé sommaire de cette étude remarquable et intéressante au plus haut point - surtout si bien docutmentéc. L'anteur avait mis sous les yeux de ses auditeurs un nombre considérable de tableaux contenant de; diasrammes, des profils et des flans dessinés spécralemenl à l'occasion de cette conférence. On en trouvera la reproduction complète dans les rolumes du Congrès. En attendant, nous pensons que ce résume donnera une idee assez fidele du beau travail de $M$. Crolard.

L'industriel qui utilise un cours d'eau provenant d'un glacier voit son usine paralysée en hiver par un gel de plusieurs semaines, tandis qu'en été l'eau est surabondante; de même, celui qui se sert d'une rivière provenant de régions moins montagneuses manque d'eau en hiver et en été, les crues l'obligent parfois à chômer. On a depuis longtemps cherché la compensation qui pourrait s'établir entre ces régimes si différents.

Si l'on considère ce qu'est cette compensation pour l'émissaire d'un lac, en particulier si l'on examine ce qui se passe pour le Thiou, émissaire du lac d'Annecy, on voit que ce dernier n'améliore guère le régime du cours d'eau. L'étude de l'allure du lac montre que chaque année, vers ia fin de l'été et en automne, le niveau descend très près du zéro des échelles hydrométriques, alors qu'aux hautes eaux il atteint la cote $1^{\mathrm{m}} 40$; les graphiques des débits de l'émissaire qui correspondent à ceux des niveaux du lac montrent qu'il y a concordance entre les débits et les variations de niveau; la régularisation n'a donc pas lieu.

Depuis 25 ans, on a créé des barrages régulateurs. Il est, par suite, intéressant de voir comment se sont réalisées les prévisions qui ont déterminé leur établissement.

C'est le Président Carnot, alors qu'il était ingénieur ordinaire à Annecy de 1866 à 1870 , qui a étudié le premier projet de régularisation du lac.

I.e Conseil municipal d'Annecy avait demandé le curage des canaux pour faciliter la navigation à vapeur|aux abords de la ville. M. Carnot proposa alors d'établir des barrages régulateurs destinés à mainterir le niveau de l'eau à la cote minimum nécessaire à la navigation et à laquelle les hautsfonds produisant des émanations malsaines en basses eaux pendant l'été, n'émergeraient plus. Sans relever le niveau des eaux lors des crues, on devait, cependant, vers la fin de celles-ci, créer une réserve capable de fournir un débit constant, jusqu'à ce que le lac atteigne la côte minimum; à ce moment le débit des émissaires, devait être rendu égal à celui des affluents.

Il s'agissait, pour résoudre ce problème, de déterminer 
d'abord l'importance des crues comme amplitude et comme durée.

Les crues ont une amplitude maximum de $1^{m}$ o6. M. Carnot, explique, par la nature des roches peu absorbantes du bassin de réception du lac, les oscillations très sensibles, à des dates très variables, de son niveau. La durée de la montée est plus courte que celle de la descente, parce que le lac reçoit par tout son périmètre et qu'il ne rend que par son émissaire.

Ne possédant pas d'autres données que l'indication des nivaux journaliers, $M$. Carnot a calculé les débits annuels à l'aide d'une série de jaugeages effectués dans les canaux. Les courbes des débits lui ont d'abord montré que ce sont les six premiers mois qui doivent constituer la réserve et que le débit moyen pour sept années, en dehors des crues, est de $9^{m 3} 9^{3}$ à la seconde. Il en a conclu que pour que le niveau du lac ne s'abaisse en aucun cas au-dessous de la cote minima fixée $(0,25)$, il fallait assurer dans les canaux un débit de $8 \mathrm{~m}^{3}$ à la seconde.

Pour remplir cette condition, $\mathbf{M}$. Carnot ava1t imaginé des vannes à débit constant, automobiles en raison de la fréquence de leur manceuvre. Ces vannes équilibrées par des contre-poids sont mues au moyen d'un flotteur, par l'intermédiaire d'un levier de longueur invariable; des galets directeurs et d'appui facilitent leur déplacement.

En construisant la courbe que décrit une de ses extrémités suivant toutes les valeurs de la différence des niveaux amont et aval, tandis que l'autre extrémité suit une droite verticale, on voit que l'enveloppe de toutes les positions que le balancier occupe dans son mouvement est le lieu des centres instantanés autour desquels il peut être supposé tourner.

Le mémoire de $M$. Carnot est un très intéressant travail d'observation et d'analyse; le conférencier rend à son auteur le grand hommage qu'il mérite.

Le 4 décembre 1876 , le ministre des travaux publics, après des expériences sur les manœuvres quotidiennes des vannes et des enquêtes diverses, approuvait un règlement pour la manœuvre des barrages régulateurs du lac d'Annecy. Or, si l'on étudie les variations de niveau du lac depuis cette époque jusqu'à ces dernières années, on ne constate pas la formation de la réserve prévue; le règlement est donc défectueux et $M$. Crolard voit la cause de cet insuccès dans le trop grand débit donné aux canaux émis. saires du lac.

Il établit, en effet, d'après les bulletins de la Commission météréologique de la Haute-Savoie, que la quantité d'eau tombée sur les 27040 hectares du bassin du lac correspond aux débits moyens de :

$\begin{array}{cccc} & 1 \mathrm{~m}^{\mathrm{m}} 858 & \mathrm{I}^{2 \mathrm{~m}^{3}} 664 & \mathrm{I}^{\mathrm{m}^{3}} 5 \text { 90 } \\ \text { pour les années } & \mathrm{I} 899 & 1900 & 190 \mathrm{I}\end{array}$

tandis que les débits moyens observés ont été de :

$$
6^{\mathrm{m}^{3}} 57^{\circ} \quad 5^{\mathrm{m}^{3}} 54^{\circ} \quad 8^{\mathrm{m}^{3}} 64^{\circ}
$$

- soit en moyenne $57,3 \%$ des débits calculés d'après l'eau tombée.

Le débit moyen pour les années en question étant de $6^{\mathrm{m}^{3}} \mathrm{go}$, on ne peut pas donner aux canaux un débit de 8000 litres à la seconde comme l'avait calculé M. Carnot.
Le chiffre de 5000 litres qui a été proposé d'après l'avis unanime des membres du Syndicat du Thiou (I), est nécessaire et suffisant. Pendant l'année igor, classée comme pluvieuse, la réserve a fonctionné six fois pour assurer le débit de 5000 litres à la seconde alors que l'alimentation du lac ne devait être en ces moments que de i 500 litres, chiffre calculé d'aprés l'abaissement du niveau qui était de un centimètre par jour. En admettant donc une hauteur d'eau de $o^{m} 65$ pour la réserve, celle-ci pourra parer à 65 jours sans pluie et si l'on tient compte de ce que pendant la moitié de cette période l'alimentation passe du minimum de I 500 litres à 3000 on trouve que le régime proposé peut parer à l'éventualité d'une sécheresse de 80 jours qui ne s'est d'ailleurs pas produite pendant les trois années consécutives indiquées précédemment.

Il importe de retenir ces deux chiffres: celui de 1500 litres comme débit des toutes basses eaux et celui de $5^{m 3}$ comme débit moyen; ils montrent que le débit des basses eaux est un peu plus du quart de ce débit moyen. De là, cette conclusion : la règlementation actuelle du lac donne aux usines qui utilisent son émissaire une puissance du triple au quadruple de la puissance minima.

M. Crolard remercie publiquement, au nom des industriels intéressés, M. le Maire d'Annecy et MM. les ingénieurs des Ponts et Chaussees de la sollicitude qu'ils leur ont témoignée dans l'effort fait par eux pour améliorer le régime de leur cours d'eau.

La réglementation admise est pourtant soumise à des conditions locales qui limitent son action. Etant reconnu que le département de la Haute-Savoie est de tous celui qui reçoit le plus d'eau túmbée annuellement, on ne pouvait guère, à cause des crues subites, relever le niveau du lac audessus de la côte $0 \mathrm{~m}$. 8o ; pour éviter leur grande amplitude le conférencier proposerait, par quelques corrections dans les canaux émissaires, de porter leur débit de $40^{m^{3}}$ à $5 \mathrm{~cm}^{3}$.

Avant de passer a l'étude plus générale de la régularisation des cours d'eau par les lacs, M. Crolard, aborde quelques considérations sur le régime des cours d'eau de la région.

Si la quantité d'eau qui tombe annuellement sur une surface donnée est à peu près constante, le débit du cours d'eau qu'elle engendre subit des écarts considérables et pour béaucoup d'entre eux il y a lieu de considérer deux étiages, celui d'été et celui d'hiver. Les plus basses eaux sont admises comme étant inférieures au $1 / 4$ des eaux moyennes; en observant que la consommation toujours variable d'une usine est le plus souvent inférieure à $40 \mathrm{o} / \mathrm{O}$ de son maximum, on en conclut que l'on utilise moins de $400 / 0 \mathrm{du} 1 / 4$ de l'eau soit moins de $1 / 10$. Si les eaux peuvent être emmagasinées dans un lac, leur utilisation peut être complète c'est-à-dire dix fois meilleure que celle habituelle.

Lorsqu'on utilise une chute créée par une eau courante, cette eau est dépensée quel que soit le nombre d'heures pendant laquelle on utilise les chevaux qu'elle produit. Avec une eau dormante, il en est autrement, elle ne coule que

(1) Groupement des industriels utilisait le Thiou. 
pendant les heures d'utilisation et ces variations de consommation pertubent le régime des émissaires du lac auxquels on demande ainsi le cheval à polonté; il y a donc lieu de rendre à ces émissaires leur débit constant. A cet effet divers systèmes de vannes automatiques ont été essayées avec succès, entre autre celle de $M$. de Chauvillerain. Toutefois pour les grands volumes d'eau comme ceux des installations sur le Rhône, la compensation entre les écarts de débit n'est pas possible.

On peut dire que les lacs s'alimentent uniquement par leurs affluents, la quantité d'eau de pluie tombée sur leur surface étant négligeable par rapport à celle reçue par leur bassin d'alimentation, quand celui-ci a une surface considérable par rapport à celle des lacs eux-mêmes. Ils n'ont le plus souvent qu'un émissaire qui peut quelquefois, à certains moments, devenir un affluent, tel le canal de Savière avec le lac du Bourget.

Les affluents comme les émissaires peuvent ètre superficiels ou sous-lacustres et accusés par des entonnoirs (lac de Joux); parfois un lac à deux sortes d'émissaires et le superficiel ne fonctionne qu'en hautes eaux. Pour chercher un émissaire sous-lacustre, on peut s'aider, soit de la température, soit de variations de débits provoquées aux points que l'on suppose être ceux d'alimentation, soit de la coloration à la fluorescéine.

Au point de vue de la régularisation il y a une grande différence entre les effets produits par l'émissaire superficiel et l'émissaire sous-lacustre. Le premier déversera d'autant plus que le niveau du lac s'élèvera, tandis que le débit du second sera très peu modifié; si les affluents diminuent l'émissaire superficiel ne débitera presque plus alors que le sous-lacustre fonctionnera toujours. Donc, tant qu'il s'agit de maintenir le niveau constant, le déversoir superficiel est un régulateur infiniment meilleur que le déversoir souslacustre.

La plus grande variation de niveau $\left(8 \mathrm{~m}_{I I}\right)$ appartient au lac Majeur. A ce sujet, le conférencier dit quelques mots des seiches étudiées par M. Forel. Les seiches sont des marées en miniature qui font osciller d'une façon rythmique le niveau des eaux; leur durée est de quelques minutes seulement et leur amplitude de 45 millimètres en moyenne pour le lac de Genève.

Sous le rapport de leur formation on peut ranger les lacs en deux classes : $1^{\circ}$ ceux qui ont leur lit dans la roche même et qui ont été formés, soit par des mouvements de l'écorce terrestre, soit par des causes d'origine externe $; 2^{\circ}$ ceux qui sont formés par un barrage, occasionné par un éboulement, la moraine d'un glacier, une coulée de lave, etc. Mais, d'après $M$. Delebecque, les lacs ne constituent dans le profil en long d'un cours d'eau qu'un accident qui, à peine formé, tend à disparaître. Les causes amenant leur disparition sont principalement: les avalanches, la végétation envahissant leur fond, les alluvions, l'émissaire abaissant la digue, etc.

M. Crolard passe ensuite rapidement en revue les différents exemples de régularisation offerts par les lacs suivants :

Lac Léman ou de Genève. - Les premiers projets de régularisation datent de 1875 et ce n'est qu'en 1884 qu'une convention intercantonale entre les états de Geriève, de Vaud et du Valais règle la question de régularisation.

Antérieurement aux travaux exécutés à cet effet le débit du Rhône variait de 50 à, $500^{3}$ à la seconde, tandis que maintenant le débit maximum est de $7^{00^{m^{3}}}$ et la variation de o m. 60 admise pour les différences de niveau extrêmes permet de porter à $100^{m^{3}}$, le débit du Rhône en hiver.

Ce lac présente une anomalie curieuse : il en sort plus d'eau qu'il ne paraît y en entrer d'après les données pluviométriques. On l'explique par le déversement dans le bassin du Rhône d'eaux provenant d'autres bassins et par l'eau de condensation provenant du givre et de la rosée sur les corps humides ou froids.

Lac de Joux (Suisse). - Il est alimenté principalement par l'Orbe; son écoulement se fait entic̀rement par des fissures appelées entonnoirs, dont la plupart sont visibles et quelques-uns utilisés comme force motrice; leur débit serait de $6^{\mathrm{m}^{3}}$ par seconde. Afin d'éviter les inondations en relevant le niveau pour créer une réserve il suffit d'un canal d'évacuation de $21^{\mathrm{m}^{3}}$. Le niveau du lac pouvant varier de la cote 1005 a la cote 1008 , on dispose d'une réserve de $35.000 .000^{m^{3}}$ donnant un débit de 1600 litres à la seconde; sous une chute utilisable de 234 mètres on a donc une puissance disponible de 3750 HP.

Mais on peut, au moyen d'un bassin régulateur à l'aval de l'usine, accrôtre l'élasticité de cette puissance; en utilisant par exemple 2600 litres par seconde, pendant 6 heures on obtiendra $6000 \mathrm{HP}$. Des travaux sont en cours d'exécution pour l'utilisation de ce lac.

Lac de Chalain (Ain). - La réserve créée au lac Chalain, par une tranche d'eau de $1 \mathrm{o}^{\mathrm{m}}$ permet de porter à $16^{\mathrm{m}} \mathrm{m}$, au lieu de $6 \mathrm{~m}^{3}$ à son étiage ordınaire, le débit du Saut-Mortier. Les travaux de captage exécutés sur les plans de M. Butticaz compurtent : une conduite de $2^{\text {mi }} 60$ de diamètre intérieur s'amorçant à dix mètres au-dessous du niveau moyen, métallique sur une longueur de 35 mètres et en béton sur 68 mètres; elle aboutit à un puits de communication pro. fond de 30 mètres qui amène l'eau à une galerie de i 70 mètres de long, taillée dans la marne dure grise; cette galerie va en remontant jusqu'à la cote 20 , au puits de berge, de là, le souterrain de Marigny de 772 mètres de long amène les eaux dans un canal ouvert de 250 mètres qui les conduit dans l'Ain.

Lac Paladru (Isère). - Les ouvrages de régularisation ne comportent qu'un déversoir et une vanne de prise dont on règle la levée suivant le niveau des eaux; pour une même cote on distribue davantage à la montée des eaux qu'à la descente. Le déversoir est une sécurité pour les crues et la vanne de prise, tant que le niveau n'est pas trop bas, donne un débit sensiblement constant.

Lac de la Girotte (Savoie). - Un projet de régularisation consiste à prendre l'eau par un tunnel et un puits à I 5 mètres au-dessous du déversoir actuel pour régulariser le Doron à 6 ou $7^{\mathrm{m}^{3}}$ par seconde au moyen d'une prise au lac de 500 à 3000 litres par seconde.

Lac Crozet (Isère). - En 1886 , ce lac a d'abord été mis en vidange par un siphon, mais le débit de 60 litres à l'amorçage tombant à 35 à 40 litres ne faisait baisser le niveau que de $7 \mathrm{~m}$. Un barrage projeté doit porter le plan 
d'eau à 7 mètres plus haut et donner un cube de $\mathrm{I}$ million 750.000 mètres soit environ les $2 / 3$ du volume total de ses deux affluents pendant l'année. Comme il y a chevauchement entre le soutirage et le remplissage, il devra y avoir, sauf dans les années très pluvieuses, très peu d'eau de perdue; le débit moyen prévu est de 95 litres par seconde.

Actuellement, la réserve est de $127000 \mathrm{~m}^{3}$ pouvant assurer un débit constant de 163 litres par seconde pendant trois mois. M. Crolard parlant du projet d'aménagement $\mathrm{du}$ bassin de Lancey par $\mathbf{M}$. Bergès termine en concluant que c'est le plus bel exemple de régularisation des cours d'eau par les lacs.

Lac des Echets (Ain), - C'est un exemple de lac artificiel faisant partie du projet de M. Pech sur l'adduction des eaux de l'Ain à Lyon. Etabli sur l'emplacement d'un ancien lac des Dombes, il devait recevoir la moitié de la réserve de r 10 millions de mètres constituée par les eaux de l'Ain qui atteignent jusqu'à $700^{\mathrm{m}^{3}}$ à la seconde. La puissance développée par le débit de $2 \mathrm{I}^{\mathrm{m}^{3}}$ avec ioo mètres de chute entre le plateau de Sathonay et les usines situées sur le Rhône et la Saône, serait de 22500 HP continus.

Lac compensateur de Jonage. - Situé vers le milieu du canal d'amenée, il a une superficie de 160 hectares. M. de la Brosse estime que ce lac permet de portcr la puissance nette de l'usine de Cusset de 16 à 20000 HP au lieu de I 2 à $16000 \mathrm{HP}$.

M. Crolard fait enfin remarquer que les lacs sont encore des modérateurs de climats par la chaleur qu'ils emmagasinent en été et dégagent en hiver. M. Forel a calculé, en effet, que pendant l'hiver 1879 -I 880 la quantité de chaleur dégagée par le lac Léman aurait été égale à celle dégagée par la consommation de 55 millions de tonnes de charbon.

Puis le conférencier termine par des considérations d'ordre pittoresque en demandant pour a ces nappes d'azur qui, hier, nous paraissaient inertes et n'avoir de vie que celle que leur donne le vent en balançant leurs flots $»$, le nom de Houille blene, comme forme allotropique de la Houille blanche.

\section{Comparaison des lacs pris comme exemples de régularisation annuelle ou journalière.}

\begin{tabular}{|c|c|c|c|c|c|c|c|}
\hline \multicolumn{2}{|r|}{ DESIGNATION } & Allitude & Surface & $\begin{array}{c}V \text { ariations } \\
\text { de } \\
\text { ivean }\end{array}$ & $\begin{array}{l}\text { Cube de } \\
\text { régularisation }\end{array}$ & OBSERVATIONS & $\begin{array}{l}\text { Puissance donnét } \\
\text { par la réserve }\end{array}$ \\
\hline 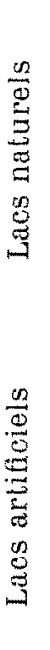 & 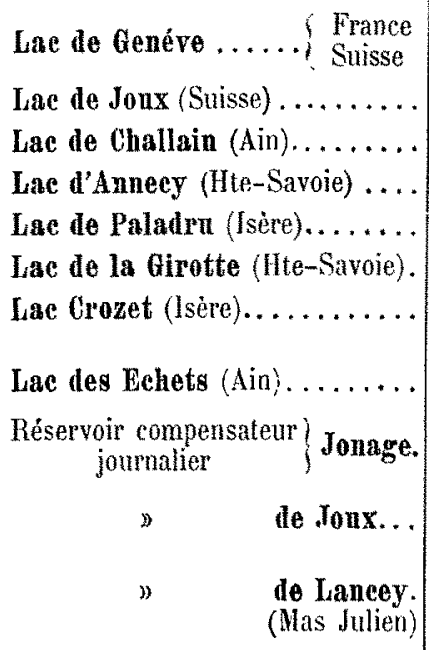 & $\begin{array}{r}372 \mathrm{~m} 28 \\
1008 \mathrm{~m} 50 \\
500 \mathrm{~m} 00 \\
446 \mathrm{~m} 52 \\
500 \mathrm{~m} 70 \\
1736 \mathrm{~m} 00 \\
1968 \mathrm{~m} 00\end{array}$ & $\begin{array}{r}582 \mathrm{~km}^{2} 36 \\
10 \mathrm{~km}^{2} 00 \\
2 \mathrm{~km}^{2} 31 \\
27 \mathrm{~km}^{2} 04 \\
3 \mathrm{~km}^{2} 90 \\
0 \mathrm{~km}^{2} 56^{8} \\
0 \mathrm{~km}^{2} 04^{8} \\
\text { moyenne } \\
16 \mathrm{~km}^{2} 00 \\
\text { maxima } \\
1 \mathrm{~km}^{2} 60 \\
0 \mathrm{~km}^{2} 05 \\
0 \mathrm{~km}^{2} 02^{8}\end{array}$ & 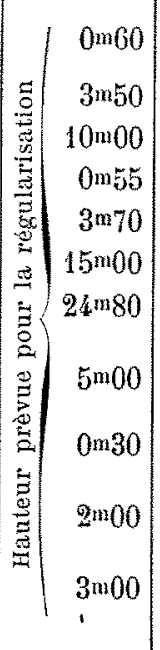 & $\begin{array}{r}349.200 .000 \mathrm{~m}^{3} \\
35.000 .000 \mathrm{~m}^{3} \\
23.100 .000 \mathrm{~m}^{3} \\
14.850 .000 \mathrm{~m}^{3} \\
14.430 .000 \mathrm{~m}^{3} \\
8.520 .000 \mathrm{~m}^{3} \\
1.150 .000 \mathrm{~m}^{3}\end{array}$ & 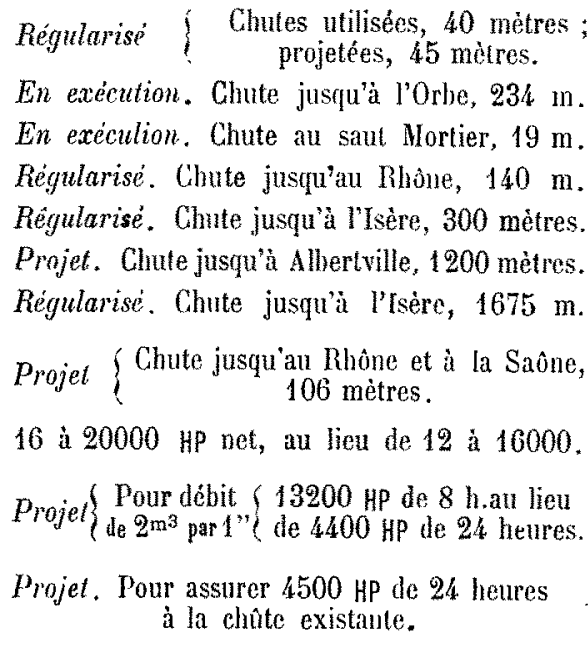 & $\begin{array}{r}38.800 .000 \mathrm{HP} \mathrm{h} \\
22.000 .000 \mathrm{HPh} \\
1.200 .000 \mathrm{HPh} \\
6.000 .000 \mathrm{HPh} \\
12.000 .000 \mathrm{HPh} \\
30.000 .000 \mathrm{HPh} \\
4.700 .000 \mathrm{HPh}\end{array}$ \\
\hline
\end{tabular}

Après M. Crolard, M. Bravet, ingénieur des Arț et Manufactures, à Chapareillan, prend la parole pour soumettre au Congrès une proposition intéressant le reboisement des montagnes.

Les lacs, dit-il, sont d'immenses réservoirs, mais il en existe une innombrable quantité d'autres, infiniments petits, qu'il ne faut pas négliger, car ce sont eux qui alimentent les grands : il s'agit de ces porosités des montagnes qui absorbent monientanément l'eau pour la rendre ensuite à la façon des grands régulateurs dont a parlé M. Crolard. Il importe de protéger ces infiniment petits.

Dans ce but, on dit aux montagnards: il faut reboiser. Mais ceux-ci répondent qu'ils ont besoin des pâturages pour faire l'élevage du bétail qui est leur principale ressource.
Le reboisement leur cause même un préjudice sı grave, que l'on a vu certains villages disparaitre par suite de l'application du nouveau régime forestier.

Or, c'est l'habitant de la plaine qui profite de l'eau, c'està-dire des avantages du reboisement; il serait donc juste qu'il offrit un revenu à l'habitant de la montagne, en compensation des dommages qui en résultent pour lui. M. Bravet pense que cette compensation devrait s'établir grâce à la formation, par bassins fluviaux, de syndicats s'étendant des montagnes à la mer. Une règlementation posée sur des bases uniformes, mais avec des variantes appropriées à chaque région, pourrait amener entre tous les intéressés, la balance des servitudes et des avantages. 


\section{CONFÉRENCE SUR LA CONSTRUCTION DES BARRAGES}

\author{
Par M. A. Dumas, \\ Ingénieur des Arts et Manulactures
}

L'autcur fait d'abord remarquer les avantages que présentent les chutes d'eau créées au moyen de barrages de retenue sur celles réalisées au moyen de canaux de dérivation. Non seulement le réservoir constitué par un barrage d'une certaine hauteur forme un excellent bassin de décantation, mais encore il peut permettre de doubler, et même de tripler, pendant quelques heures de la journée, le débit du cours d'eau, c'est-à-dire la puissance disponible de l'usine, a condition, naturellement, de restreindre cette puissance pendant les heures où son utilisation ferait d'ailleurs défaut.

M. Dumas passe ensuite rapidement en revue les divers modes de construction de barrages-réservoirs et projette sur un écran un certain nombre de profils de ces ouvrages constituant des types auxquels peuvent se ramener tous les barrages existants. Il fait ressortir les avantages et les inconvénients de chacun de ces divers systèmes, en insistant sur les vices de construction que présentent certains d'entre eux et qu'on doit avoir le souci d'éviter.

En ce qui concerne les barrages en maçonnerie, M. Dumas rappelle les progrès qu'a fait, depuis une dizaine d'années, la théorie d'après laquelle ils doivent être établis. Le plus essentiel a été la constatation que, dans ces ouvrages, la maconnerie devait ètre, à tout prix, soustraite aux efforts d'extension, c'estad-dire que la courbe des pressions ne devat jamais sortir du tiers médian de la section de l'ouvrage.

Apres avoir signalé les travaux des ingénieurs qui ont, dans ces dernières années, apporté des contributions intéressantes à l'étude de ces questions, en particulier, ceux de M. Maurice Lévy, membre de l'Institut, M. Dumas donne quelques indications pratiques sur la construction dés grands barrages. Il appelle l'attention sur la nécessité qu'il y a à revêtir la face en contact avec l'eau d'un enduit aussi imperméable que possible et donne quelques détails sur des essais tout récents, effectués sur divers ouvrages.

\section{ETUDE RAISONNÉE DU MEILLEUR SYSTÈME DE PRISE D'EAU DANS UN TORRENT}

\author{
Conférence de M. Drouhin
}

Directeur général de la Société électrochimique de la Romanche.

Le conférencier, laissant de côté toutes considérations théoriques, et s'appuyant exclusivement sur les résultats pratiques obtenus à ce jour, se propose de rechercher le meilleur mode de captage d'un torrent.

Prenant comme type le systeme de dérivation établi à Livet sur la Romanche, qui fonctionne depuis trois ans sans avoir jamais donné lieu à aucun mécompte, M. Drouhin examine les conditions auxquelles doit satisfaire une prise d'eau. Il faut, dit-il:

Io Eviter l'encombrement de la prise par les apports constants du torrent.

$2^{\circ}$ Constituer, à l'amont de la prise, par une retenue appropriée, un bassin de décantation où la vitesse de l'eau étant ralentie, celle-ci se débarrassera de la plus grande partie des matières qu'elle tient en suspension.

$3^{\circ} \mathrm{N}$ 'admettre dans les canaux d'amenée que les eaux de surface, pour compléter la décantation.

$4^{\circ}$ Construire le barrage de retenue de telle façon que les crues n'aient pas d'action sensible sur l'ouvrage, c'est-àdire en évitant les effets de la sous-pression produite à l'aval et sous l'ouvrage par l'eau retenue en amont, ainsi que les affouillements dûs à la chute; fractionner l'ouvrage pour qu'en cas de rupture, le niveau amont ne s'abaisse pas au point de causer une interruption de service.

50 Soustraire le canal d'amenée aux accidents de montagne: avalanches, trombes, chutes de rochers; lui donner une pente suffisante pour permettre l'évacuation rapide des matières en suspension dans l'eau et ménager sur son parcours de nombreuses chasses afin d'obtenir des eaux bien décantées dans la chambre aval.

$6^{\circ}$ Disposer cette chambre pour que, en cas d'accident à l'usine, on puisse fermer l'admission des turbines, sans danger pour la conduite torcée, et sans être obligé de vider le canal d'amenée.

$7^{\circ}$ Enfin, protéger la conduite forcée contre les effets de la mise en charge et de la vidange et la chûte des blocs qui pourraient la perforer.

Ces conditions étant ainsi définies, le conférencier passe en revue les ouvrages et organes qui doivent y satisfaire.

Barrage de retenme. - Les barrages de grande hauteur ne paraissent pas justifiés sur les torrents, encombrant rapidement de leurs apports le bassin amont, lequel d'ailleurs, est, dans bien des cas, de peu d'utilité au point de vue de l'effet compensateur; il suffit de 5 à 6 mètres de hauteur pour opérer la décantation et la chasse des apports. La masse d'eau retenue à l'amont, désagrège le fond du lit en chassant le sable qui agglutine les galets et si un barrage de grande hauteur n'est pas fondé sur le rocher ou à très grande protondeur dans le lit $d$ 'a torrent, les effets de sous-pression peuvent compromettre la résistance de l'ouvrage. Or, cet ouvrage sera toujours d'un établissement coûteux. Mieux vaut donc constituer une faible retenue.

En plan, le barrage doit avoir la forme d'une courbe dont le rayon dépend de l'écartement des contreforts de butée qui jouent le rôle de culées de voûre, la convexité faisant face au sens du courant. Le sectionnement de l'ouvrage, en vue de limiter les dégâts en cas de rupture, sera obtenu au moyen d'arceaux intermédiaires (1) - calculés d'après la méthode

(1) Le barrage de Livet, d'un développement de 30 mètres, est divisé en trois arcs formant voussoirs appuyés sur des pillers de $10 \mathrm{~m} \times 3 \mathrm{~m}$; l'un des bajoyers du pertuis et l'ancrage dans les berges de la rive droite forment culées. L'ensemble présente en plan la forme d'un arc de cercle de $60 \mathrm{~m}$. de rayon à la crête du barrage.

On a exécuté d'abord les piliers, puis les arceaux latéraux et enfin l'arceau central, De cette façon le barrage a pu être facilement exécuté et si jamais il venait béder, ce ne pourrait être que sur un des arceaux ce qui n'entrainerait pas de chômage. 
graphique de Méry sur la stabilité des voûtes - opposant chacun sa résistance propre à la poussée: ils s'appuieront sur des piliers et le tout sera englobé dans la forme finale de l'ouvrage à l'aide de remplissages en béton de ciment.

Pour éviter les affouillements à l'aval de l'ouvrage on constituera un matclas d'eau de $1 \mathrm{~m}$. à $1 \mathrm{~m}$. 50 de hauteur, suffisant pour amortir la chute; à cet effet, la courbe supérieure du profil transversal sera la parabole d'écoulement, correspondant aux plus fortes crues, raccordée tangentiellement à une contre-courbe, elle même tangente au lit du torrent en aval du barrage.

Pertuis et radier de chasse. - Sa nécessité s'impose pour l'évacuation des apports continus du torrent. En le munissant de vannes, on peut, d'une part, retenir tout le débit en basses eaux et, d'autre part, créer, en les soulevant un peu durant quelques minutes, des courants de fond qui déblaient rapidement le radier s'il s'encombre. Celui-ci, dont la pente sera de $o \mathrm{~m} .03 \mathrm{~cm}$. par metre afin d'obtenir un fort courant de chasse, sera établi à un niveau inférieur à celui du lit en amont du barrage pour créer sur ce point un appel de tous les apports.

Déversoir latéral de prise d'eau. - Le système de prise à gueule-bée, trop souvent adopté, présente beaucoup d'inconvénients, entre autres l'obstruction du canal d'amenée par les apports, ce qui occasionne des chòmages, et l'insuffisance de la décantation de l'eau franchissant la prise, ce dont les organes distributeurs souffrent grandement.

Aussi, le conférencier préfère-t-il le système de prise en déversoir n'admettant que des eaux de surface partiellement décantées. La crête de ce déversoir devra se trouver au moins à deux mètres au-dessus du radier du pertuis afin de n'être jamais affleurée par les apports; l'épaisseur de la lame déversante, en basses eaux, devra être telle que tout le débit du torrent soit absorbé par le déversoir, le niveau de l'eau retenue ne devant pas dépasser la crête du barrage. On évitera les remous qui obstruent les grilles par le dépôt des corps flottants, en disposant le déversoir de prise sur le prolongement amont du bajoyer du pertuis adjacent à la rive.

Chambre de prise d'ean. - Elle est indispensable pour régulariser l'admission de l'eau dans le canal d'amenée, augmenter la décantation et servir d'appui à la grille d'arrêt des corps flottants ainsi qu'à la vanne de prise du canal d'amenée; son cube doit être d'au moins quinze fois le débit à assurer pour éviter les tourbillons sous la vanne de prise. Une bonde de fond devra permettre l'évacuation des matières décantées.

Canal d'amenée. - Suivant la disposition des lieux, il sera à ciel ouvert, souterrain, ou formé de tuyaux. En tous cas, la vitesse de l'eau devra y être de 2 mètres à la seconde (pente de $15 / 10 \mathrm{~m} / \mathrm{m}$ ) au maximum si les parois ne sont pas revètues de maçonneric; dans certains cas, on aura même intérêt à réduire cette pente pour augmenter la chute; si, autrement, il doit en résulter une économie dans le coût des travaux, on pourra admettre des vitesses de 3 à $3 \mathrm{~m} 50$ par seconde.

On couvrira le canal aux points dangereux ou même on l'établira en tunnel pour l'abriter contre les avalanches.

A fin d'éviter l'affouillement produit par l'ouverture de la vanne de prise ainsi que le soulèvement du radier par souspression, on constituera, à l'aide d'un petit barrage établi à 10 ou 15 mètres a l'aval de la vanne de prise, un matelas d'eau de $0 \mathrm{~m}$. 50 à $0 \mathrm{~m}$. 60 de hauteur qui, ayant pour effet d'amortir la vitesse au moment oú commence l'ascension de la vanine, s'opposera aux effets précédents.

Dans un canal souterrain, il est prudent de revêtir d'une chappe de béton, même le rocher compact, afin d'éviter les pertes par les failles; les plus insignifiantes lorsqu'elles sont pénétrées par pression peuvent occasionner des effets désastreux. Sur tout son parcours on aura la précaution de ménager des chambres de chasse pour compléter la décantation.

La section rectangulaire est préférable pour les canaux à ciel ouvert, tandis que la forme circulaire devra être employée dans les souterrains et pour les conduites fermées en ciment armé.

Les conduites en tôle doivent être enterrées sur la moitié au moins de leur section pour empêcher leur déformation au moment de la mise en charge. Il conviendra d'aménager, à très faible distance de la vanne de prise, un puits de visite et d'aspiration si le canal est en souterrain, ou un large reniflard si la conduite est fermée, en ciment ou en tôle. En outre, dans ce cas, on disposera, sur divers points du parcours, des tubes piézométriques formant cheminées et ayant pour effet de parer aux coups de bélier.

Chambre d'eau aval. - Cet ouvrage qui domine généralement l'usine l'expose à de graves dangers en cas de rupture; il doit être établi avec tous les soins possibles. $M$. Drouhin est d'avis de le diviser en deux parties: une chambre de décantation et une chambre de travail. Elles doivent être séparées par un batardeau surmonté de vannes verticales, pouvant les isoler complètement. Les sables décantés dans la première sont expulsés par des vannes de fond; les vannes d'admission dans la seconde ont pour effet de laisser toujours la conduite forcée, vide ou pleine, en communication avec l'atmosphère, ce qui remplace avantageusement les reniflards et permet, comme un tube piézométrique, d'amortir les coups de bélier, tandis qu'une vanne noyée ou à cloche, fermant l'orifice de la conduite, n'offrirait pas ces avantages.

La chambre de décantation sera munie à ses extrémités de deux vannes de vidange, puis d'une grille à barreaux serrés ou mieux,d'une tôle perforée, pour arrêter les feuilles. Enfin, l'un des murs sera disposé en déversoir capable éventuellement de débiter tout le cube dérivé ; ce dispositif dispense d'opérer la vidange complète du canal d'amenée à l'usine et procure l'économie d'un homme préposé à la mancuvre des vannes à la prise d'eau.

Le conírencier préconise une chambre de travail indépendante afin d'isoler, brusquement s'il le faut, le canal d'amenée; les vannes d'admission dans cette chambre pourraient même ètre commandées électriquement de l'usine. Son cube devra, pour qu'il ne s'y produise pas de remous, être au moins égal a i 5 fois le débit par seconde.

Conduite forcée. - C'est l'organe qui doit être calculé avec le plus de soin; on sait que sa rupture a occasionné la destruction d'usines.

Il ne faut pas que les tôles en acier doux travaillent à 
plus de 5 à 6 kilogs par $\mathrm{m} / \mathrm{m} 2$. A Livet, où la chute est de 60 mètres, M. Drouhin n'a admis que 4 kilos.

Comme on le sait, la résistance à la rupture est fonction du diamètre et le constructeur devra toujours largement tenir compte des efforts anormaux des coups de bélier. Les conduites en ciment résistent à des charges de 20 mètres, mais le conférencier préfère la tôle d'acier Martin.

Pendant la mise en charge, la conduitc tendant à s'ovaliser, il faudra, pour la maintenir cylindrique, l'armer ou l'enterrer sur moitié de sa hauteur; dans les parties verticales, il faut parer à la poussée au vide par un solide encastrement des coudes adjacents.

On a vu précédemment les précaution à prendre pour amortirles coups de bélier et éviter l'effet du vide.

Dans les terrains à pic où les chutes de rocher sont fréquentes, on devra, malgré l'excédent de dépense, loger la conduite, soit dans un puits comme à Livet, soit dans une tranchée complètement remblayée. Faute de cette précaution un bloc tombant de haut sur la conduite peut la percer comme un emporte-pièce.

Un autoclave et une vanne de vidange et de chasse sont des appareils qu'il est bon de disposer sur la conduite; même il pourra être intéressant d'y adapter un manomètre enregistreur des pressions.

Enfin M. Drouhin conseille de disposer les prises des turbines au-dessus de la conduite parce que les joints des tubulures en $1 / 4$ de cercle sont plus accessibles que ceux placés entre la conduite et les turbines sur des tubulures droites lorsque les prises sont latérales.

$$
* *
$$

M. A. Boucher, ingénieur à Prilly (Suisse), membre du Conseil d'administration du Syndicat des Forces hydrauliques, chargé de compléter la série des communications sur l'Hydrologie et l'Hydraulique, prend la parole après M. Drouhin. Mais, pressé par le temps, il ne fait qu'effeurer les points suivants.

L'étude hydrologique d'un bassin de montagne montre qu'il existe une différence entre l'eau que ce bassin reçoit d'une façon apparente et cellequi en sort par son émissaire: cette anomalie provient non seulement des évaporations mais aussi des condensations occultes.

Lorsqu'on fait une prise d'eau il faut se préoccuper de tous les corps flottants, notamment des glaces flottantes et des glaces de fond.

La décantation des sables, dans les ouvrages de dérivation d'un cours d'eau, est presque toujours d'un effet illusoire; les purges continues seules sont réellement efficaces pour obtenir des eaux plus ou moins purifiées.

\section{CONFÉRENCE \\ SUR LES INSTALLATIONS ÉLECTRIQUES}

\author{
Par M. R. $-V$. Picov \\ Ingénieur-électricien.
}

M. Picou parle des trois personnalités en présence dans l'aménagement d'une chute : le propriétaire, l'hydraulicien et l'électricien; c'est de leur entente que dépend la réussite de l'installation. Ordinairement les choses se passent de la façon suivante :

Le propriétaire s'adresse d'abord à l'hydraulicien pour l'inviter à formuler des propositions. Ce dernier propose alors, de toutes les turbines pouvant satisfaire aux conditions de hauteur et de débit de la chute, celle qui est la moins coûteuse, c'est-à-dire celle qui a la plus grande vitesse angulaire. En faisant autrement, il craindrait de se mettre dans une position d'infériorité vis-à-vis ses concurrents.

Ce n'est qu'à ce moment que l'on fait intervenir l'électricien; le calcul de la dynamo à réaliser se trouve pour lui défini par la nature du courant à produire, et par la vitesse angulaire de la turbine arbitrairement fixée sans son avis. Or, très souvent, cette vitesse angulaire trop grande est incompatible avec les exigences d'une bonne construction électrique. La solution logique serait de réclamer du proprietaire le choix d'une turbine à moindre vitesse, afin de pouvoir y atteler une dynamo remplissant exactement les conditions électriques imposées.

Mais cette solution est rarement adoptée, car turbine et dynamo augmentant de dimensions et par conséquent de prix, le propriétaire ne manquerait pas de s'adresser au concurrent qui fait des offres plus avantageuses en utilisant la turbine prévue. Et pour ne pas manquer l'affaire, l'électricien accepte sans mot dire les conditions imposées, se fiant à la chance pour résoudre les difficultés qui lui sont ainsi créées.

Comment donc établir l'entente entre ces trois personnes? - Par un ingénieur-conséil, à la fois mécanicien et électricien, qui aura la haute direction de l'installation. Mais le rôle de l'ingénieur-conseil, incompris du propriétaire, est en outre autant que possible évincé par les constructeurs.

Dans ce cas, c'est l'électricien qui doit avoir le dernier mot sur les conditions d'établissement de la turbine. L'usine demande, en effet, à être établie d'abord dans les conditions techniques les plus favorables et ensuite le plus économiquement possible; le meilleur prix qu'il importe d'obtenir est beaucoup plus celui du kilowatt-heure d'énergie électrique que celui du kilowatt de puissance installée. Or c'est l'électricien qui est le plus apte à juger des dispositions à adopter pour atteindre ce but.

Celui-ci étant chargé de la direction générale doit avant tout s'efforcer d'appliquer ces deux formules : pas de tours de force; pas de luxe.

Chercher à obtenir un rendement inutilement élevé est un tour de force; que le rendement des dynamos soit de 0.88 ou $0.9^{2}$, une exploitation n'en est jamais modifiée de façon bien appréciable; il faut avant tout faire robuste et durable.

La tendance à la génération directe des hautes tensions est aussi plutôt regrettable. Sans compter qu'elle n'est pas toujours économique, car les dimensions de la machine s'exagèrent avec la tension, il faut considérer la différence de sécurité contre les coups de foudre que présente l'une ou l'autre des combinaisons.

La deuxième formule - pas de luxe - s'applique surtout aux tableaux de distribution. Ils doivent être, non pas des « panneaux d'exposition of faits pour flatter l'œil et comportant une série d'appareils inutiles au surveillant, mais bien réellement des tableaux de service où les seuls instruments 
de mesure qui doivent y figurer, sont ceux dont les indications servent à déterminer la manœuvre d'un appareil de réglage ou de coupure. Ce n'est pas à dire que certains appareils complémentaires, tels que des enregistreurs ne puissent donner des indications utiles à l'exploitation, mais ceux-là doivent être dans le bureau de l'ingénieur ou du directeur et non pas sur le tableau.

M. Picou termine sa conférence en faisant ressortir toute l'importance pratique de ces diverses considérations.

\section{CONFÉRENCE SUR LA RÉGULARISATION DE LA VITESSE DES TURBINES}

\author{
Par M. Ribourt \\ Professeur à l'Ecole centrale des Arts et Manufactures.
}

M. Ribourt, ingénieur, professeur de construction des machines hydrauliques à l'Ecole centrale, rappelle que, dans une des conférences précédentes de ce Congrès, M. Picou, l'éminent électricien, a demandé que les conditions d'établissement des turbines hydrauliques soient subordonnées à celles des machines électriques destinées, comme intermédiaires obligatoires, à transposter aux points d'utilisation l'énergie de la houille blanche.

Parmi ces conditions, la constance de l'allure de ces machines, pour le facteur vitesse, paraît essentielle et doit même comporter, dans bien des cas, une précision de premier ordre, tandis que l'autre facteur de l'énergie, l'effort tangentiel développé, peut subir des variations importantes, avec les mêmes écarts que ceux de la puissance à utiliser.

Ce résultat important est depuis longtemps déjà obtenu par les régulateurs automatiques dont la plupart des turbines sont pourvues. Ces appareils modifient, en fait, le débit de l'eau actionnant la machine et sont pour cela placés sous la dépendance d'un système mécanique en équilibre par rotation: le pendule centrifuge, qui agit dès que la vitesse s'écarte du régime normal.

Il est intéressant de fixer les divers éléments de ce problème important de la régulation d'une installation mécanique commandée par une turbine, par une figuration graphique ; un exemple en est placé sous les yeux du Congrès pour montrer l'origine du mouvement ondulatoire qui s'établit et se prolonge indéfiniment avec des amplitudes qui dépendent non seulement de la perfection du systeme employé, mais aussi de l'importance des masses tournantes composant l'installation et de la puissance d'action du mécanisme de manœuvre du vannage de la turbine.

La sensibilité du pendule centrifuge, universellement employé jusqu'ici, a paru suffisante pour réduire les écarts de vitesse dont il s'agit; mais, pour certains cas, il peut être intéressant d'obtenir mieux encore.

M. Ribourt a cherché à résoudre ce problème avec un appareil nouveau qui s'affranchit du pendule centrifuge et présente une sensibilité beaucoup plus considérable.

C'est par une fonction hydraulique que cet appareil agit, en présentant cette particularité qu'on peut déterminer à volonté le degré de sa sensibilité, suivant les applications.
M. Ribourt montre au Congrès les dispositions essentielles de son régulateur, arce des graphiques théoriques représentant la loi de rupture d'équilibre qui le caraciérise, comparée à celle du pendule centrifuge. Des résultats entièrement pratiques sont présentés au Congrès par des relevés faits sur une installation mise $\epsilon \mathrm{n}$ expériences prolongées et dans des conditions particulièrement difficiles, dont l'exécution a été confiée aux ateliers des constructeurs bien connus MM. Teisset, Breult et Chepron, à Chartres.

Malgré des variaticns imfortantes de la résistance, le courant produit par cetle ir s1allaticn actionnant un atelier de travail des bois, les variations de la vitesse sont remar$q$ uablement réduices et à feinesensibles, four ainsi dire,sur ces diagrammes.

\section{$*^{* *}$}

A la suite de cetie conférence, $\mathbf{M}$. Sloan, ingénieurconstıucteur, à Paris, fait remarquer combien les industriels exploitant des forces bydrauliques, ou les propriétaires qui se proposent d'aménager des chutes d'eau sont mal outillés, soit pour évaluer le rendement des turbines qu'ils possèdent, soit pour vérifier les garanties proposées par les constructeurs et il invite les membres du Congrès à suivre l'exemple des Américains qui ont créé une station d'essais d'appareils hydrauliques.

Cette station serait pourvue de tous les appareils nécessaires pour effectuer, avec précision, les mesures de débit, de variation de charges, de vitesses, de rendement, etc... Un personnel d'ingénieurs spécialistes lui serait attaché pour effectuer ces mesures, soit à la station, soit au dehors, dans les usines des industriels. Propriétaires et construc. teurs, chacun serait ainsi certain de trouver des garanties dans l'emploi d'appareils perfectionnés, aux mains d'opérateurs expérimentés.

Cette proposition eñ fait naître une autre, discutée par plusieurs membres du Congrès, tendant à réunir les propriétaires d'appareils hydrauliques en une association semblable à celles des propriétaires d'appareils à vapeur et même à les faire entrer dans ces dernières, en y formant des sections spéciales.

Ces propositions, ayant été prises en considération, ont fait l'objet de deux vœus adoptés par le Congrés et que nous avons reproduits dans notre précédent numéro.

\section{CONFÉRENCE DE M. THURY}

\author{
Directeur de la Compagnie \\ de l'Industrie Electrique et Mécanique de Genève.
}

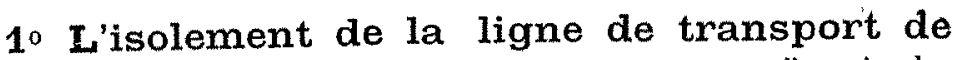
force de Saint-Maurice à Lausanne, d'après les essais officiels exécutés les $g$ et 10 septembre 1902.

On sait l'importance considérable de la question de lisolation des lignes industrielles à très haut voltage. La distance que l'on peut atteindre, c'est-à-dire la longueur de ces lignes, dépend en grande partie de cet isolement et comme l'on recherche nécéssairement, de plus en plus, l'aug- 
mentation de rette distance, tout ce qui se rattache à ce problème est utile à connaître.

Beaucoup d'essais ont été déjá faits en employant comme courant d'épreuve de l'alternatif à très haute tension; quelques personnes ont poussé leurs expériences jusqu'à I 00000 volts et plus.

Mais, il n'a pas été fait des essais sérieux à haut voltage continu. Comme l'emploi de ce genre de courant semble appelé au plus grand avenir pour les transports de force, il y a intérêt à faire connaître ce que l'on peut en attendre. Voici les résultats des essais officiels de la ligne de SaintMaurice-Lausanne, exécutés ces jours derniers, épreuve pour laquelle on a fait usage de courant continu à 20000 volts.

La ligne du transport de force de la commune de Lausanne a maintenant une longueur simple de 58 kilom. Elle comprend un conducteur de 116 kilomètres supporté par 1500 poteaux en bois de sapin injecté et 3000 isolateurs à double cloche du modèle Ginori, employé sur la ligne triphasée Parderno-Milan. Ce modele a été étudié par M. Semenza, ingénieur de la Société Edison italienne.

Comme on le sait, cette ligne doit transporter 5000 chevaux de Saint-Maurice à Lausanne, au moyen d'un courant d'intensité constante, continu, et de voltage variable.

C'est l'emploi du continu,comme moyen de transport,qui motive l'essai de la ligne au continu, car on ignorait si à 20000 volts et plus, il ne se produirait pas quelque phénomène insoupçonné.

Le matériel d'essai comprenait une petite dynamo de 25 kilowatts à courant continu, excitée séparément et capable de donner directement toutes les tensions désirces jusqu'à 25000 volts, à la condition que l'intensité ne dépassàt pas 1,5 ampère. Comme instruments de mesure, il a été fait usage d'un voltmètre apériodique de Hartmann et Braün, grand modele de précision, genre Desprez d'Arsonval, et un milli-ampèremètre de précision dont l'échelle proportionnelle porte i 50 divisions pour 30 milliampères, du genre Weston à glace. Le voltmètre étair monté en dérivation et l'ampèremètre e s série sur le fil allant à la ligne.

Les essais ont été faits d'abord entre les deux fiis de ligne, puis entre les deux lignes réunies et le sol, successivement avec le pôle $(+)$ à la ligne ou le pôle $(-)$. La durée de l'électrisation, d'un essai au suivant, était supérieure à une minute. Elle a été maintenue 30 minutes pour l'essai à 20000 volts.

Ces expériences ont donné les résultats suivants:

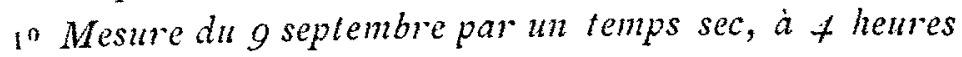
après midi.

Moyenne des mesures à 20000 volts.

Intensité du courant $=0$, o I I ampère.

Résistance totale de l'isolement $=1802000 \mathrm{ohms}$.

Résistance de l'isolement par kilm $(58)=104,5$ mégohms,

Energie consommée : total 220 watts; par isolateur $=$ 0,073 watt;

En \% de l'énergie transmise normalement : $0,07 \%$.

Résistance d'isolement entre le sol et les deux,lignes réunies en parallele à 20000 volts : (a) Pôle (一) à la ligne : Résistance isolement gériéral = 0,877 mégohm.

Par isolateur : $263 \mathrm{r}$ mégohms.

(b) Pôle $(+)$ à la ligne : Résistance isolement géréral = 0,740 mégohm.

Par isolateur 2220 mégohms.

$2^{\circ}$ Essais du ro septembre à $g$ heures du matin, par un fort brouillard sur toute la ligne: Entre fils.

Moyenne de ro mesures à 20000 volts:

Intensité du courant : o, o 3 ampere.

Résistance de l'isolement général $=1540000 \mathrm{ohms}$ (soit les 0.85 de l'isolement par temps sec).

Energie consorméc $=260$ watts. Par isolateur $0,0866^{\circ}$ watt.

En \% de l'énergie transmise normalement : 0,09 .

Résistance d'isolement entre le sol et les dcux lignes réunies en parallèle (temps humide) le pôle (-) à la ligne.

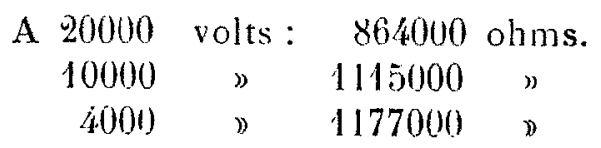

Isolément mesuré entre le sol et les deux lignes réunies, le pôle (-) étant réuni à la ligne :

$\begin{array}{rccc}\text { A } 20000 & \text { volts : } & 882000 & \text { ohms. } \\ 10050 & " & 1082000 & \nu \\ 4080 & " & 1180000 & " \\ 21100 & " & 1250000 & \end{array}$

L'isolement diminue au fur et à mesure de l'augmentation du voltage. Cet effet est très peu sensible dans les mesures entre conducteurs. Il est plus marqué dans les mesures entre le sol et les conducteurs réunis.

Dans les essais du 9 septembre, l'isolement entre fils et sol était de i 316000 ohms à 5000 volts. Il est descendu à 877000 ohms, à 20000 volts, le pôle (-) à la ligne.

Ces résultats sont très encourageants, car ils démontrent que les pertes en ligne par dérivation, pertes par l'air, etc., sont absolument négligeables avec le courant continu au voltage déjà passablement élevé de 20000 volts, et cela sur une ligne à gros fil de 58 kilomètres de longueur. Il est à noter que les 3000 isolateurs de cette ligne étaient loin d'être tous en parfait état : une visite rapide en avait révélé environ 20 cassés et ébréchés par les pierres lancées par des enfants. La ligne, pendant les essais, était donc bien à l'état normal, c'est-à-dire telle qu'elle est en pratique journalière. Les essais auraient eu moins de valeur s'ils avaient été effectućs sur une ligne fraichement revisée, sans un seul isolateur brisé, c'est-à-dire telle qu'elle n'est pas en pratique.

Il faut aussi tenir compte que l'isolement des tableaux de six réceptrices et celui des parafoudres est compris dans les mesures. Pour simplifier, on a préféré ne pas les découpler de la ligne, afin de ne pas perdre un temps précieux.

La perte pourrait donc être cent fois plus forte avant d'atteindre même le I \%. La marge pratique est considérable.

Si l'on rapproche ces résultats de ceux obtenus avec le courant alternatif, on est, au premier moment, assez surpris de la différence en faveur du continu. Mais, au fond, tout s'explique par la différence des voltages, à voltage efficace 
égal, et par l'absence complète des effets de capacité, produisant des phénomènes de résonnance souvent fàcheux.

On ne peut parler d'électrolyse des isolateurs : il n'y a rien et il ne peut rien y avoir à craindre de ce côté là, car les courants dérivés sont infinitésimaux, donc impuissants à électrolyser la porcelaine vitrifiée.

\section{$2^{\circ}$ Emploi de la terre comme conducteur pour les courants puissants.}

Jusqu'ici, la terre n'a pas été utilisée comme conducteur de courant, sauf pour les faibles intensités usagées en télégraphie, téléphonie, signaux, etc. Dans la traction électrique même, la terre ne joue quaccidentellement le rôle de conducteur, car la majeure partie du courant passe par les rails, et l'on préférerait même que la totalité de l'ampérage veuille bien suivre cette voie, sans aucune dérivation par le sol, afin d'éviter les nombreux ennuis que cause l'électrolyse aux conduites d'eau et de gaz. Ces inconvénients sont parfois très graves et préoccupent tellement les éiectriciens qu'à première vue, l'emploi de la terre pour la transmission régulière de courants industriels peut paraître basardé.

M. Thury a voulu se rendre compte si réellement la terre ne pouvait pas rendre pratiquement d'aussi bons services en matière de transport de force à grande distance, qu'en télégraphie. Il a donc procédé à quelques essais pratiques sur une installation industrielle, et il en expose les très intéressants résultats.

Grâce à l'obligeance des ingénieurs du Sérvice électrique de la commune de Lausanne, il a pu utiliser, à cet effet, le transport de force de Saint-Maurice à Lausanne, lequel se prêtait particulièrement bien à cette expérience.

La distance à vol d'oiseau entre les usines génératrice et réceptrice est de 56 kilomètres. La force motrice est empruntẹe au Rhône, à Saint-Maurice, et les cinq mille chevaux comprenant la première période doivent pouvoir être transmis dans leur totalité à l'autre extrémité de la ligne, c'est-à-dire à Lausanne.

Par raison d'économie et de sûreté, l'emploi du courant continu à intensité constante a été choisi par la commission d'experts de la commune; cette intensité est de 150 ampères; elle est indépendante de la charge. Le voltage varie entre 2000 et 22500 volts, proportionnellement à l'énergie transmise.

La ligne actuelle est composée de deux conducteurs de $150 \mathrm{~m} / \mathrm{m}^{2}$ chacun. Sa résistance totale est de $13,3 \mathrm{ohms}$ à $20^{\circ}$ et la perte normale est de 2000 volts, soit de $9 \%$ de l'énergie disponible à la sortie de l'usine génératrice. Cette ligne pèse environ 152 tonnes, soit 30 kilngs par cheval sur l'arbre des turbines.

Le problème consistait à réduire la ligne à un seul conducteur, le second étant remplacé par la terre ellemême. Si la terre ne présentait pas de résistance, la perte et le poids de cuivre utile seraient réduits de moitié, soit 15 kilogs de cuivre par cheval, et $41 / 2 \%$ de perte à pleine charge; ou, si l'on veut, à perte égale, le cuivre serait réduit au $1 / 4$, soit $71 / 2$ kilogs par cheval, chiffre extrêmement bas pour 56 kilomètres de distance de transport. Mais, en pratique, cela n'a pas été tout à fait le cas.
Avec une seule ligne, soit 15 kilogs par cheval au licu de 30 , la perte tutale a été de 1210 volts, soit $5,5 \mathrm{o} / \mathrm{o}$. Le gain sur le transport ordinaire tel qu'il fonctionne actuellement, était donc d'une part l'économie de la moitié du cuivre, soit 76 tonnes, et d'autre part une économie d'énergie de 790 volts $\times 150$ ampères, soit 118500 watts. Ce résultat est donc encourageant.

La résistance du sol érait exactement $\mathrm{I}, 4$ ohm au total, entièrement attribuable au défaul du contact entre les plaques de terre et le sol, et à la résistance de ce dernier dans le voisinage immédiat des points de mise à la terre. La distance proprement dite n'entre absolument pour rien dans cette résistance, et $M$. Thury croit pouvoir affirmer que si les deux points de mise à la terre eussentété placées, non pas à 56 kilometres l'un de l'autre, mais aux antipodes, le résultat eut été exactement le même.

La mesure individuelle de la résistance de chacune des deux plaques de terre a donné les résultats suivants:

A Saint-Maurice, perte à 150 ampères $=185$ volts. $\mathrm{R}=1,233 \mathrm{ohm}$. A Lausanne, perte à $150 \mathrm{amp}=25$ volts. $\mathrm{R}=0,167$. La différence s'explique par le fait qu'à Lausanne, l'expérimentateur a fait usage des terres des parafoudres lesquelles sont réunies aux conduites d'eau de l'usine au moyen d'un fil de cuivre, tandis qu'à SaintMaurice la terre était constituée par un conducteur composé de rails usagés, enfouis le long de l'usine dans un sol graveleux qui s'est révélé très résistant.

Malgré cela, à 50 mètres de distance environ le sol était déjà presque au même potenticl qu'à Lausanne.

A Saint-Maurice, l'usine est bâtie au fond ae la vallée du Rhône, assez resserrée en cet endroit. Elle est fondée sur les bords du cone d'alluvion du Bois Noir.

A Lausanne, l'usine est bàtie, comme toute la ville, sur un banc de molasse, à 605 mètres d'altitude, soit à 230 mètres au-dessus du lac Léman.

La perte totale a été mesurée directement au voltmètre entre l'origine des deux terres (bornes des génératrices et réceptrices), en utilisant à cet effet la ligne de 150 millimètres disponible du fait de l'emploi de la terre à sa place. La résistance individuelle de chaque mise à la terre a été mesurée au moyen d'un piquet métallique approprié, fiché en terre à une distance suffisante. La somme des deux pertes s'est exactement contrôlée par la mesure directe effectuée au moyen de la ligne libre employée comme fil pilote.

Ces essais résolvent-ils le problème? Non; mais c'est un premier pas en avant vers une solution simple doublant sensiblement la distance de transport à perte égale et permettant l'emploi d'un seul fil, solution idéale. Toutefois bien des essais sont encore nécessaires avant d'aborder les applications pratiques.

Au point de vue de l'électrolyse, $M$. Thury estime que la solution la plus radicale sera l'emploi, pour le pôle positif d'électrodes en charbon de cornue, coke, etc., de nature indestructible. Pour le pôle négatif, c'est naturellement le fer qui conviendra le mieux.

Pour éviter les effets fâcheux des courants vagabonds le long des conduites d'eau et de gaz, il suffira de réaliser des terres rationnellement réparties sur une zone assez étendue. 
On ne se contentera donc pas d'une terre unique, localisée à une faible étendue de terrain; le mieux sera d'établir pour chaque pôle un certain nombre de terres rayonnantes, aussi profondément enfouies que possible et embrassant une zone d'autant plus étendue que le terrain sera plus rocheux et moins conducteur. Les conduites d'eau et de gaz ne peuvent être attaquées que si elles réunissent entr'elles des portions du sol entre lesquelles règnent des différences du potentiel appréciables. Il est facile de déterminer expérimentalement ces différences par leur recherche au moyen d'un voltmètre et de piquets de terre. On les équilibrerd ensuite au moyen de lignes et plaques de terre appropriées.

La mise à la terre d'un pôle peut occasionner des différences de potentiel élevées entre les enroulements et les bâtis lorsque ceux-ci sont isolés du sol. L'expérience a démontré que ces différences de potentiel peuvent s'équilibrer avec facilité au moyen de l'intercalation de très fortes résistances reliant les bâtis avec les enroulements, en pratique supérieures à I mégohm. Ces différences ne se manifestent que lorsque la résistance des isolements des enroulements est elle-même extrêmement grande.

\section{SUR LE CALCUL RAPIDE DES CONDUCTEURS AÉRIENS AU MOYEN D'UNE ABAQUE UNIQUE}

(Mémoire de M. Blondel résumé par M. Gullbert)

Le travail de M. A. Blondel oftre un grand intérêt pour le calcul des canalisations aériernes, car il permet d'abréger considérablement les tâtonnements inévitables pour le choix du diametre des fils, la distance des supports et la flèche admissible.

L'auteur rappelle, tout d'abord, les deux formules utilisées pour le calcul des lignes : celle relative au calcul de la flèche :

$$
f=\frac{p x^{2}}{8 T}=\frac{\pi x^{2}}{8 T}
$$

et celle relative aux changements des conditions :

$$
\alpha\left(\theta-\theta^{\prime}\right)=\frac{\mathbf{x}^{2}}{24}\left[\frac{\boldsymbol{\pi}^{2}}{\mathbf{T}^{2}}-\frac{\boldsymbol{\pi}^{\prime 2}}{\mathbf{T}^{\prime 2}}\right]-\frac{\mathbf{T}-\mathbf{T}^{\prime}}{\mathbf{E}}
$$

ou : $\mathbf{x}$ est la distance horizontale entre les supports.

E le coefficient d'élasticité du métal des conducteurs.

$\mathbf{T}$ et $\mathbf{T}^{\prime}$ les tensions en $\mathrm{kg}: \mathrm{mm}^{2}$. au point le plus bas de la chaînette pour les deux températures $\theta$ et $\theta^{\prime}$.

$\alpha$ le coefficient de dilatation.

f la flèche.

* la pression uniformément répartie (poids propre, poids du verglas et pression du vent) rapportée également à la section et exprimée en $\mathrm{kg}$ par mètre de longueur.

p la pression totale par $\mathrm{mm}^{2}$ de section.

Après avoir passé en revue les méthodes connues jusqu'ici pour simplifier lescalculs, M. Blondel expose la sienne qui consiste à construire pour chaque métal employé les courbes de la formule:

$$
y=\alpha 0=\frac{\pi^{2} x^{2}}{24 T^{2}}-\frac{T}{E}
$$

en fonction de la variable $\mathbf{x}$ et pour des valeurs constantes de la tension, en indiquant sur les courbes les valeurs correspondantes de la flèche.

Cette indication des valeurs des flèches permet d'obtenir un second réseau de courbe, $\mathbf{f}=\mathbf{C}^{t e}$, en réunissant par des courbes continues les points correspondants à une même flèche.

Comme on le voit, l'équation (3) contient encore un paramètre qui est la pression $\pi$ ce qui nécessiterait la consttrucion des deux séries de courbes pour chaque valeur de $\pi$ Pour conserver les mêmes courbes quelle que soit la valeur de $\pi, M$. Blondel use d'un artifice ingénieux et basé sur cette remarque que les seconds membres des équations (1) et (2) subissent des changements équivalents quand on multiplie par un même facteur soit $\pi$ seulement, soit à la fois $\mathbf{f}$ et $\mathbf{x}$ en laissant $\boldsymbol{\pi}$ constant.

Au lieu de modifier $\boldsymbol{\pi}$, on peut donc conserver les mêmes réseaux de courbes en modifiant $\mathbf{f}$ et $\mathbf{x}$, c'est-à-dire en remplaçant la flèche et la distance horizontale des supports par d'autres semblables avec un rapport de similitude égal au facteur par lequel on aurait multiplié le poids spécifique $\pi$, pour tenir compte de son augmentation.

Les poids spécifiques introduits par $\mathrm{M}$. Blondel sont des poids fictifs puisqu'ils tiennent compte de la pression du vent. Pour les déterminer et mieux, pour calculer le rapport $\frac{\pi}{\pi}$ du poids spécifique fictif au poids réel, $M$. Blondel compose le poids spécifique réel avec la pression du vent supposée horizontale et s'exerçant sur la surface cylindrique du fil.

La valeur du poids $\pi$ ' est alors :

$$
\pi^{\prime}=\sqrt{\pi^{2}+\frac{1}{\mathrm{~s}^{2}}(0,001 \times 0,57 \times \mathrm{Pd})^{2}}
$$

$\mathbf{P}$ étant la pression du vent par mètre carré de surface plane, $\mathbf{s}$ la section et $\mathbf{d}$ le diamètre.

Les courbes annexées au travail de $M$. Blondel se rapportent au bronze silicieux; elles sont construites pour des tensions allant jusqu'à $20 \mathrm{~kg}: \mathrm{mm}^{2}$ et pour des portées se pro: longeant jusqu'à 300 mètres, de façon à permettre le calcul des efforts du vent comme il vient d'être dit.

\section{CONFÉRENCE SUR LE TRANSPORT DE L'ÉNERGIE}

Par M. Borssonnas,

Ingénieur, Directeur de la Société Franco-Suisse pour l'Industrie Electrique.

M. A. Boissonnas, dans sa communication, passe en revue les principaux systèmes utilisés pour le transport de l'énergie électrique.

A la suite des essais si intéressants faits récemment à St-Maurice sur le transport de force en série, suivant les dispositions adoptées par M. Thury, il établit un parallèle 
entre ce système et les transports par courant alternatif triphasé.

L'exemple choisi comporte le transport à 200 kilomètres d'une force d'environ 4000 chevaux à 40000 volts, et passant en revue les phénomènes secondaires attachés à ce genre de distribution, "le conférencier arrive à la conclusion qu'au point de vue technique les deux systèmes offrent leurs avantages propres et que seules des conditions spéciales peuvent, dans chaque cas, faire donner la préférence à l'un ou à l'autre.

Au point de vue économique, l'exemple choisi semble représenter la limite que l'on peut aujourd'hui pratiquement atteindre si, à une distance de 200 kilomètres, on veut soutenir la concurrence des machines à vapeur et des moteurs à gaz. (1)

La question du retour par la terre avec courant continu n'a pas été prise en considération dans cet exposé, car elle n'est pas encore sortie de la période d'essais et ne pourra être envisagée que lorsque les pouvoirs publics se seront prononcés à cet égard.

M. Boissonnas termine en disant que la lutte entre le transport de force électrique et les machines à vapeur ou les moteurs à explosion devient toujours plus ardente. Elle s'explique, malgré la souplesse et les avantages de l'électricité, lorsquau vu d'exemples vécus on considère que la force naturelle est bien éloignée de signifier force gratuite et lorsque l'on sait que le transport de force a trois ennemis implacables dont les effets nuisibles croissent plus vite que la distance à franchir : le ciel, la terre et les hommes, sous formes de la foudre, des branches d'arbres et de la malveillance qui sont toujours aux aguets pour troubler une exploitation.

Le transport électrique de l'énergie est donc encore un être délicat, car il a rapidement grandi et il a besoin de toute la sollicitude et des égards des pouvoirs publics pour survivre à ses concurrents et étendre encore son champ d'action.

M. Boissonnas invite ensuite les membres du Congrès, au nom de la Société Grenobloise de Force et Lumière, à asıister à quelques essais d'isolateurs à haut voltage croissant jusqu'à 70000 volts, dans son laboratoire de Grenoble, afin de juger des effets électriques et lumineux qui s'attachent à l'emploi des hautes tensions.

Disons de suite que ces essais très ingénieusement organisés ont présenté le plus vif intérêt pour les spectateurs; nous en reparlerons plus loin.

\section{CONFERENCE DE M. A. GODINET}

Ingènieur civil à Lyon.

M. Godinet, pour traiter de l'éclairage, fait l'hrstorique tout à fait général des entreprises de gaz et de celles d'électricité. Il constate que les premières ont eu, après des débuts extraordinairement difficiles, une existence particu-

(1) Nous publierons prochainement à ce sujet l'étude très documentée qui nous a été remise bien avant le Congrès par l'un de nos collaborateurs. lièrement heureuse, mais que les derniers contrats passés avec les municipalités comportent des prix si bas que ces affaires sont à éviter. Il dit, notamment, qu'une ville qui voudrait réaliser une entreprise d'éclairage au gaz, créée de toute pièce, ne pourrait pas faire face au service de l'emprunt qu'elle aurait consacré à cette entreprise, si elle appliquait, vis à vis de ses abonnés et d'elle-même, les conditions inscrites dans les cahiers de charges les plus récents. Le conférencier, disant de l'éclairage électrique qu'il n'a pas eu, en général, de jours heureux, recherche les causes de ce fait. L'une des principales, à ses yeux, est que l'on a fait des prix de vente trop bas, et conclu les traités de ces dernières années à des conditions dangereuses, ne laissant pas de marge pour avoir des bénéfices aver un amortissement suffisant. Faisant un rapprochement entre l'industrie de l'éclairage et l'industrie des chutes d'eau, il cherche à montrer aux membres du Congrès qui se sont réunis pour discuter sur la législation à appliquer aux chutes d'cau, que dans cette industrie nouvelle, il faut s'attendre à des imprévus, à des mécomptes, à des difficultés de placer sa force immédiatement, et il conclut en demandant au législateur des lois libérales, encourageant une industrie difficile et ne l'écrasant pas avant son éclosion.

\section{CONFÉRENCE SUR LA TRACTION ÉLECTRIQUE}

\author{
Par M. Petit \\ Ingénieur, Directeur de l'Omnium Lyonnais, des Chemins \\ de fer et Tramways.
}

M. Petit montre d'abord le développement considérable de la traction électrique, particulièrement en Amérique où l'on compte à ce jour 35000 kilomètres de lignes et 52000 voitures automotrices. En France, le mouvement a été également très important. Ainsi en 1900, la traction électrique comprenait plus de 1100 kilonètres de lignes, soit le quart de l'ensemble des lignes de tramways en exploitation. Depuis, on a construit le Métropolitain de Paris, la ligne de Versailles aux Invalides (Compagnie de l'Ouest), la ligne de la gare d'Austerlitz à la gare d'Orsay (Compagnie d'Orléans) et la ligne du Fayet à Chamonix (Compagnie P.-L.-M.).

Le développement de cette industrie s'explique d'abord par les qualités précieuses des moteurs électriques, surtout pour le démarrage et les surcharges, puis par la centralisation de la production en une seule station de l'énergie nécessaire à l'exploitation, d'où un rendement plus élevé et des frais d'exploitation moindres.

Le conférencier adopte ensuite la classification suivante: 10 Lignes de tramways urbains et de banlieue.

$2^{\circ}$ Lignes de chemins de fer vicinaux ou d'intérêt local, et lignes d'intérêt général à faible ou moyenne vitesse.

$3^{\circ}$ Lignes Métropolitaines à grand trafic, à plate-forme indépendante souterraine ou aérienne.

$4^{\circ}$ Lignes d'intérêt général à grande vitesse.

10 Lignes de tramways urbains.

Des départs fréquents, des arrêts et des démarrages 
rapides, ainsi que des vitesses peu élevées (20 à 25 kil. à l'heure), telles sont les conditions que l'on doit réaliser dans ce cas et qui ont fait adopter, presque partout, l'emploi du moteur série à courant continu, dont les qualités répondent si bien à ces exigences.

Le service est alors assuré par des automotrices isolées contenant de 35 à 50 places, et actionnées presque toujours par deux moteurs dont la puissance individuelle varie de 25 à 30 chevaux et monte même jusqu'à 50 chevaux pour les lignes très accidentées.

Le courant continu, généralement fourni à la tension de 500 à 600 volts, est recueilli dans le tramway pardes systèmes très nombreux, mais que l'on peut ramener néanmoins a trois types principaux qui sont :

$1^{\circ}$ Les lignes à trolley et fil aérien, universellement employées pour les lignes établies en dehors des villes.

Leur installation revient à 10000 ou 15000 francs le kilomètre.

$2^{\circ}$ Les lignes à canivean souterrain. - Elles sont composées d'un caniveau oủ sont logés deux conducteurs isolés chargés d'amener le courant à la voiture par l'intermédiaire d'un frotteur double. Ce dernier plonge dans le caniveau par une rainure longitudinale existant tout le long de la ligne.

Le premier dispositif employé parmi les nombreux systèmes à caniveau, est celui de Smith appliqué en Angleterre vers 1885. Le caniveau Siemens et Halske vient ensuite; il a été établi successivement à Budapest sur 60 kilomètres, puis à Berlin et à Vienne.

En France on peut citer surtout la Compagnie générale Parisienne de Tramways avec 22 kilomètres de ligne à caniveau, et la Compagnie des Omnibus et Tramways de Lyon avec 4 kilomètres environ. Ces caniveaux sont du système Thomson-Houston.

Afin de conserver un bon isolement, malgré l'humiditédu caniveau et l'entretien assez restreint de celui-ci, la tension existant entre chacun des fils d'amenée de courant et la terre n'est que de 250 volts tout en étant de 500 volts entre les deux fils.

Les frais d'exploitation des lignes à caniveaux sont peu supérieurs à ceux des lignes aériennes. Le prix de l'installation, par contre bien plus élevé, atteint 150000 à $250000 \mathrm{fr}$. le kilomètre de voie simple.

30 Les lignes à contact superficiel. - Le courant est amené à la voiture par l'intermédiaire de plots placés dans l'entrevoie et qui, par un dispositif spécial, ne reçoivent le courant qu'au moment où ils sont recouverts par le frotteur de la voiture.

Le système Claret-Vuillemier est le plus ancien, mais le plus employé est le «Diatto " qui malgré les inconvénients qu'il a présentés au début à Paris, surtout à cause de la déformation des appareils par la circulation intense, compte déjà en France r 30 kilomètres de voie en service.

Les frais d'installation des lignes à contact superficiel varient de 60000 à 80000 francs par kilom. de voie simple.

- Pour les lignes extra-urbaines, oú le trolley est presque toujours employé, le rayon d'action des stations centrales à courant continu, sous 500 volts, est limité à 8 ou ro kilomètres. On éloigne un peu cette limite en alimen. tant les points extrêmes à des tensions plus élevées par des moteurs survolteurs et des feeders spéciaux.

Le système à trois fils avec les rails pour conducteur neutre ne paraît pas devoir prendre beaucoup d'extension en raison de la difficulté que l'on éprouve à équilibrer les deux ponts. Il en est de même du dispositif utilisé sur la ligne de Grenoble à Chapareillan où l'on emploie du courant continu à rooo volts, avec deux lignes aériennes, sans employer les rails comme conducteur de retour, en raison des complications qu'entraîne ce système pour la ligne aérienne.

M. Petit insiste alors sur la nécessité d'assurer un éclissage électrique parfait dans le cas du retour par les rails afin d'éviter les effets d'électrolyse. En France une prescription administrative limite à I volt par kilomètre la perte de charge dans les rails. On est alors conduit, dans les réseaux importants, à employer des feeders isolés pour le retour du courant et même, pour compenser la résistance de certains feeders isolés trop résistants, à faire usage de sous-volteurs.

\section{$2^{\circ}$ Lignes de chemins de fer vicinaux ou d'intérêt local et lignes d'intérêt général à faible on moyenne vitesse}

Les démarrages étant ici moins fréquents, le moteur série à courant continu, dont la supériorité réside surtout dans son couple de démarrage près de six fois supéricur au couple normal, ne s'impose plus aussi impérieusement. Le moteur à courant polyphasé qui, outre sa robustesse, présente l'avantage de conserver une vitesse peu variable avec la charge, qualité précieuse dans les lignes accidentées, peut alors être employé avec avantage, principalement si la ligne est longue, car les sous-stations de transformation deviennent très simples et il est, de plus, possible d'employer des moteurs marchant directement sous 3000 volts.

Comme type d'installation de chemin de fer n'employant que le courant continu, M. Petit, cite, à titre documentaire seulement, celui de l'Isar-Thal construit en r 89 I.

Lignes utilisant des moteurs à conrant continu alimentés par des usines à courant polyphasé. - Ces installations sont très hombreuses en Amérique. En Europe, la plus importante est celle de Milan - Gallarati - Varèze, qui est alimentée par une usine hydro-électrique produisant du triphasé à 13000 volts et 25 périodes. Cinq sous-stations transforment ce courant en continu à 650 volts qu un rail isolé, établi tout le long de la voie, est chargé de conduire aux voitures.

Lignes utilisant des moteurs à courant polyphasé. - Le conférencier décrit à ce sujet deux installations :

Celle de Burgdort-Thunn dont la ligne, longue de 42 kilomètres, est alimentée par une usine hydro-électrique donnant du triphasé à 16000 volts et 40 périodes. Tous les 4 kilomètres des transformateurs abaissent la tension à goo volts. Deux fils aériens, ainsi que les rails, servent à conduire le courant. Les automotrices ont 4 moteurs de 8 o chevaux; le réglage de la vitesse s'obtient par l'insertion de résistances.

La seconde installation est celle de la Valteline, en Italie, dont la ligne a ro5 kilomètres. Le courant, envoyé de l'usine à 15000 volts et 15 périodes, est abaissé tous les Io kilomètres pour être envoyé directement à 3000 volts 
dans les moteurs. Les voitures sont munies de 4 moteurs, deux de 150 chevaux et deux de 75 ; au démarrage, les moteurs de 150 chevaux recoivent seuls le courant à haute tension et on envoie dans le stator des deux autres moteurs le courant à faible tension, produit dans le rotor des deux premiers. Des résistances liquides permettent, en outre, de graduer les vitesses. Les moteurs de $7^{5}$ chevaux ne sont utilisés que pendant le démarrage.

Lignes d'intérêt général à faible ou moyenne vitesse. Les dispositions employées sont les mêmes que dans les cas précédents.

On ne compte, en France, que trois lignes de ce type: celle de Versailles aux Invalides; celle de la Compagnie d'Orléans; celle du Fayet à Chamonix.

Pour les lignes de cette catégorie, la suciété d'Oerlikon préconise le courant monophasé qui, amené à haute tension sur la locomotive, y est alors abaissé et transformé en continu par un groupe moteur-génératrice.

\section{Lignes Métropolitaines}

On se retrouve ici à peu près dans les mèmes conditions que dans le cas des tramways urbains. Le moteur série à courant continu redevient alors le seul pratiquement utilisable.

Dans le Métropolitain de Paris, que M. Petit prend comme type, l'usine centrale à vapeur produit du continu à 600 volts pour alimenter la partie du réseau voisine de l'usine, et du courant alternatif à 5000 volts et 25 périodes destiné à être transformé en continu à l'autre extrémité du réseau. Les automotrices sont munies de deux moteurs de I oo chevaux. Un Mulliple-IInit-système permet ì un seul mécanicien de commander tous les noteurs répartis sur les diverses voitures motrices. La dépense spécifique par tonne kilomètre est de 6 I watts-heure, à l'usine.

\section{$4^{\circ}$ Lignes à Grande Vitesse}

M. Petit rappelle d'abord les expériences fameuses effectuées entre Zossen et Marienfeld par la Maison Siemens et Halske et «l'Allegemeine Electricitats Gesellschaft ».

Trois conducteurs situés latéralement à la voie amenaient le courant triphasé à 10000 volts, avec 45 à 50 périodes.

Les voitures des deux maisons allemandes étaient divisées en trois compartiments dont les deux extrêmes, réservés aux voyageurs, contenaient 56 places. Au milieu se trouvaient les transformateurs et les appareils pour la mise en marche et les freins. Ces voitures dont le poids variait de 90 à 95 tonnes étaient portées par deux boggies à trois essieux, dont deux seulement actionnés par des moteurs de 250 chevaux. La prise de courant était constituée par trois archets latéraux; dans la voiture de l'Allgemeine un rhéostat liquide servait pour le démarrage; dans celle de l'autre compagnie c'était un rhéostat métallique; le mécanicien pouvait, du reste, dans cette dernière, faire varier la tension en couplant les transformateurs, soit en étoile, soit en triangle.

L'état de la voie fit que l'on dut limiter la vitesse à 160 kil. au lieu d'atteindre celle de 200 kil. qui figurait dans le programme.

Les essais, qui ont très bien réussi, montrent surtout la supériorité des moteurs polyphasés dans l'obtention des grandes vitesses et la possibilité de recueillir du courant à rooso volts par simple frottement.

Traction tangentielle. - Ce système très original dû à MM. Dulait, Rosenfeld et Zeleny n'est encore qu'à l'essai.

Le long de la voie sont placées des bobines enroulées autour de pièces polaires et qui constituent le stator d'un immense moteur polyphasé qu'on aurait, en quelque sorte, fendu suivant une de ses générations et développé sur le plan de la voie; on crée ainsi un flux magnétique glissant suivant l'axe de la voie. Sous la voiture se trouve un pro. pulseur pouvant être considéré comme le rotor du précédent moteur que l'on aurait aussi développé sur un plan.

Les inventeurs ont pu rémédier assez bien à l'inconvénient d'avoir, entre le stator et le rotor, un entre-fer relativement considérable. D'autre part, pour réduire les frais d'établissement, ils divisent le stator en une série de plots magnétiques au licu de le rendre continu.

\section{Traction électrique des bateanx sur les cananx on sur les rivières.}

M. Petit décrit deux applications importantes qui ont donné d'excellents résultats.

L'une, en Belgique sur le canal de Charleroi a Bruxelles, où les tracteurs sont actionnés par des moteurs polyphasés; l'autre, en France, sur les canaux du Nord, près de Douai, oú l'on a employé le courant continu.

M. Petit souhaite qu'en France un systeme de concession régulière soit établi, afin de permettre à cette industrie de se développer.

En terminant, le conférencier montre le vaste champ d'exploitation qui s'ouvre pour la traction électrique, appelée à remplacer, sans doute, tous les autres modes de traction. M. Petit s'étend alors sur les difficultés, surtout financières, que présente, pour le moment, cette transformation qui ne peut s'opérer que progressivement. Les usines hydro-électriques trouverunt là, un débouché important, surtout maintenant. que les sociétés concessionnaires ont le droit d'acheter leur énergie à des tiers; on sait que la loi de 1880 , avant que l'Etat ait eu modifié sa jurisprudence à l'occasion de la concession du tramway de Grenoble à Chapareillan, les obligeait à produire elles-mêmes leur force motrice. Enfin, le conférencier discute la question du prix de l'énergie électrique et il donnne à ce sujet quelques chiffres intéressants sur son prix de revient lorsqu'elle est. engendrée par la vapeur.

Avec du charbon de i 5 à 20 francs la tonne, pour des stations utilisant des groupes de $1000 \mathrm{HP}$ et au-dessus, le $\mathrm{K} w$-heure revient de ofr.04 a ofr. 06 ; pour des stations utilisant des groupes de 300 à $600 \mathrm{Hp}$, ce prix est de ofr. 06 à o fr. 08 ; et pour des petites stations utilisant des groupes de 100 à $200 \mathrm{HP}$, il atteint ofr. 08 à ofr. 10 . 


\section{L'ETAT ACTUEL DE L'INDUSTRIE ELECTROCHIMIQUE}

\author{
Confírence de M. Gall
}

Administrateur-Délégué de la Société d'Electrochimie.

Définitions générales. - Le conférencier commence par définir ce que l'on entend par ces expressions : Electrochimie, Electrométallurgie, Electrolyse.

L'Electrochime est la science qui, d'une manière générale, s'occupe des applications de l'Electricité à la Chimie. Le courant électrique agit sur les corps de deux façons différentes: soit comme agent de décomposition, soit comme un agent calorifique accumulant dans un espace restreint une grande somme d'énergie. Dans le premier cas, on fait de l'Electrol.yse; le second cas est celui des Fours ÉLECTRRUES.

L'Electromítaliurge est la branche de l'electrochimie qui concerne plus spécialement l'extraction des métaux, leur affinage, la préparation de leurs alliages et de leurs carbures.Ces opérations s'effectuent suivant deux méthodes principales : la méthode par voie humide, dans laquelle les corps sont soumis à l'action du courart électrique à l'état de sels dissous; la méthode par voie sèche, dans laquelle les matières sont traitées à l'état anhydre et fondues par l'action d'un foyer étranger ou par l'effet du courant électrique luimême. Dans la méthode par voie humide, on fait toujours de l'électrolyse; le bain contenant les sels en dissolution s'appelle l'électrolyte et les pôles par lesquels entre et sort le courant électrique s'appellent des électrodes. Le pòle posinif est désigné sous le nom d'anode et le pôle négatif sous le nom de cathode. Il peut égalęment y avoir électrolyse dans le cas de la méthode par voie sèche,'mais, plus généralement, les réactions entre les corps s'opèrent ici grâce à l'état de fluidité auquel ils ont été portés par le courant électrique lui-même, qui fournit d'ailleurs toute l'énergie nécessaire à l'accomplissement de ces réactions. Ce sont alors les fours électriques qui servent à ces opérations.

Théorie de l'Electrolyse. - Pour qu'un corps soit susceptible d'être électrolysé, il taut qu'il soit d'abord conducteur' de l'électricité et ensuite qu'il se présente sous un état de fluidité suffisante. Un électrolyte ne se comporte pas par rapport au courant électrique comme un conducteur métallique; tandis que ce dernier n'éprouve au passage aucune modification durable,si ce n'est qu'une fraction de l'énergie électrique se transforme en chaleur, l'électrolyte, au contraire, est décomposé par le courant en ses éléments constituants, suivant des lois définies. Ces éléments prennent le nom de ions; les uns se portent à l'anode, ce sont les anions, les autres à la cathode, ce sont les cathions.

Deux hypothèses servent à expliquer les phénomènes de la décomposition électrolytique. La première, celle de Clausius, émise en 1846 , suppose que les molécules des électrolytes, sont animées d'un perpétuel mouvement de translation et peuvent échanger entre elles les jons dont elles sont formées, toutes les fois que ces ions, passant à portée les uns des autres, sont chargés d'électricité de signe contraire. Le courant électrique, traversant l'électrolyte, aurait alors pour cffet d'exercer une action directrice sur les ions, au moment précis où ceux-ci passent d'une molécule à une autre. D'autre part, l'attraction des pôles sur les ions chargés d'élertricité de signe contraire serait plus forte que la force d'affinité qui les liait dans la molécule; celle-ci étant décomposée et chaque ion mis en liberté, l'hypothèse explique que ceux-ci se dirigent vers leurs électrodes attractives pour s'y dégager.

La deuxième hypothèse, due à Arrhénius, beaucoup plus récente, puisqu'elle date de 1887 , admet que la majeure partie du composé soumis à l'action électrique n'est pas à l'état normal, mais à l'état dissocié, dans l'électrolyte. Par exemple, dans une solution de chlorure de sodium, il y aurait très peu de molécules $\mathrm{Na} \mathrm{Cl}$, mais beaucoup d'jons $\mathrm{Na}$ et de ions $\mathrm{Cl}$, à l'état libre. Cette dernière hypothèse qui s'appuie d'ailleurs sur certaines théories de la chimie moderne, rend compte d'un plus grand nombre de phénomènes dans le détail desquels nous ne pouvons suivre ici le conférencier.

Nous dirons, toutefois, qu'il donne encore quelques explications sur le phénomène connu sous le nom de transport des ions, c'est-à-dire dans lequel tout se passe comme si les ions électro-positifs se transportaient vers la cathode et les ions électro-négatifs vers l'anode avec des vitesses différentes, et il cite à ce sujet les récents travaux de M. Hittorf, qui ont apporté une grande clarté à cette partie de la théorie de l'électrolyse.

Principales Industries Electrochimiques. - M. Gall consacre quelques mots à la production industrielle de l'Hrdrogène. Le procédé le plus employé est celui de M. le commandant Renard, dont les appareils, comportant des électrodes en fer, électrolysent l'eau avec une solution au $1 / 6$ de soude caustique et sous une différence de potentiel de 3 volts environ. Ces appareils peuvent supporter un courant de 400 ampères et fournissent à l'heure 8o litres d'oxygène et $\mathrm{r} 60$ litres d'hydrogène : la quäntité d'énergie absorbée est de I, 60 cheval-heure. En somme, le rendement de ce procédé est de 50 litres d'oxygène et 100 litres d'hydrogène par cheval-heure. Mais la préparation électrolytique de l'hydrogène n'a pas reçu un grand développement industriel, ce gaz, ainsi produit,étant principalement destiné au gonflement des ballons militaires. Toutefois, il était intéressant de la mentionner, en raison des applications que ces gaz de l'eau peuvent trouver par la suite dans la préparation de certains produits chimiques.

On a essayé d'employer le courant électrique à la concentration de l'acide sulfurique. Cette opération par les procédés de chauffage usuels est très difficile parce que l'on ne peut employer que des récipients en matière inattaquable: verre, porcelaine, etc., ou platine qui est très cher ; dans ces conditions, la chaleur est mal utilisée, ou bien l'on est obligé d'avoir un matériel très coûteux. En envoyant un courant électrique dans l'acide à concentrer on réalise un chauffage direct; la différence de potentiel doit être insuffisante pour produire l'électrolyse. Mais l'énergie électrique même étant produite par la houille blanche est encore plus coûteuse que le chauffage au charbon le plus économique et 
le procédé électrique ne peut devenir pratique que s'il permet de réaliser de grandes économies sur le matériel.

L'industric la plus importante dans cette branche de l'Electrochi.nie est l'électrolyse du chlorure de sodium. On la fait à trois points de vue différents : $1^{0}$ pour la fabrication de la soude caustique et la production du chlore gazeux; $2^{\circ}$ pour la préparation des hypochlorites ou liqueurs de blanchiment ; $3^{\circ}$ pour la fabrication des chlorates.

Sonde électrolytique. - La préparation de la soude caustique et du chlore s'opère par des appareils électrolyseurs qui, selon leur principe, peuvent se ranger en trois classes. La première comprend les électrolyseurs qui opèrent la décomposition du chlorure de sodium en dissolution dans des cuves électrolytiques, séparées en plusieurs compartiments par des diaphragmes en matières poreuses : terre de pipe, amiante, tissus silicatés, ciment poreux, verre pilé, ou bien par des faisceaux de tubulures en fer, en porcelaine ou autres matières. L'un des compartiments contient la cathode (pôle négatif); c'est dans celui-ci que le sodium libéré, réagissant sur l'eau, la décompose, s'empare de l'oxygène pour former la soude caustique qui reste en dissolution tandis que l'hydrogène libre se dégage à la cathode. L'autre compartiment renferme l'anode (pôle positif), à laquelle vient se dégager le chlore. Le diaphragme a pour but d'empêcher la réaction du chlore sur la soude caustique produite, tout en permettant le transport des ions au sein de l'électrolyte. Les électrodes sont généralement en charbon de cornue ou en fer et elles affectent les dispositifs les plus divers.

Dans ces appareils, les diaphragmes se détériorent ou s'obstruent assez vite et accroissent la résistance opposée au courant électrolyseur, ce qui a pour effet de diminuer le rendement. En outre, ils n'empêchent pas complètement la réaction du chlore sur la soude donnant naissance, dans l'électrolyte, à de l'hypochlorite de soude qui, d'une part, attaque rapidement les électrodes et, d'autre part, absorbe en pure perte une partie du courant pour sa décomposition. Mais, malgré ces inconvénients, différents systèmes d'électrolyseurs de cette catégorie ont puêtre mis en fonctionnement industriel d'une façon très satisfaisante.

Au nombre de ceux-ci sont les appareils OutheninChalandre que la Société La Volta a mis en service depuis un certain temps déjà dans ses usines de Moûtiers; puis, les appareils de Hargreaves et Bird employés à la Motte-Brun.

Les appareils de la deuxième catégorie sont basés sur un principe tout différent ; ils ne comportent pas de diaphragmes. L'électrode négative y est constituée par une couche mobile de mercure à laquelle vient s'allier le sodium mis en liberté à cette électrode. L'amalgame mercure-sodium, alors soumis à l'action de l'eau en dehors de l'électrolyseur ou dans un compartiment spécial de l'appareil, se décompose en donnant de la soude caustique et de l'hydrogène et le mercure régénéré fait retour à la cathode. Le chlore dégagé à l'électrode positive est recueilli par les méthodes usuelles. Les meilleurs appareils de ce genre sont ceux de Kellner et de Castner employés dans plusieurs usines en Angleterre et en Allemagne. Ils ont l'inconvénient d'employer le mercure qui coûte actuellement fort cher et dont les pertes dans les amalgames successivement formés et décomposés sont l'origine de dépenses appréciables influencant teaucoup les prix de revient. Il faut cependant reconnaître que ce procédé supprime la formation des hypochlorites ce qui amène un meilleur rendement et une plus longue conservation des électrodes.

Dans cette catégorie d'appareils, on peut ranger le procédé Hulin qui, à la place du mercure, emploie du plomb fondu et électrolyse le chlorure de sodium fondu; l'amalgame plomb-sodium est ensuite décomposé par la vapeur d'eau. Ce procédé très élégant n'a malheureusement pas donné dans la pratique les résultats qu'il pouvait faire espérer.

La troisième catégorie comprend des électrolyseurs récemment mis en essai en Allemagne et qui ne comportent ni diaphragmes ni mercure. Leur principe est basé sur la différence de densité qui existe entre la solution de chlorure de sodium électrolysée et celle de soude caustique produite. Cette dernière, plus dense, se forme au fond de l'appareil et, a l'aide de cloches convenablement disposées par rapport aux électrodes et à la couche de séparation des deux solutions, on recueille séparément et sans troubler l'équilibre de cette couche,le chlore et l'hydrogène.

De tous les appareils actuellement mis en fonctionnement il se dégage ce résultat économique que la préparation électrolytique de la soude et du chlore est plus économique que la fabrication par les anciens procédés Leblanc ou Solvay, surtout lorsque l'énergie électrique est produite par des chutes d'eau. Les rendements correspondent à une production de 1260 grammes de soude caustique et 1325 grammes de chlore, soit 400 litres par heure, avec un courant de 1000 ampères, sous une différence de potentiel de 5 volts au maximum, ce qui représente une dépense de $51 / 2$ chevaux par kilogramme de soude produite à l'heure.

Il semblerait donc que cette industrie doive se développer beaucoup. Mais, si l'on remarque que le principal emploi pratique connu du chlore est de se combiner à la chaux pour former l'hypochlorite de chaux, on voit qu'à la production de une tonne de soude correspond la fabrication de plus de deux tonnes d'hypochlorite de chaux. L'emploi de la soude est pour ainsi dire illimité, il s'en consomme en France environ 180.000 tonnes par an, tandis que l'hypochlorite de chaux, qui n'est guère employé que pour le blanchiment de la pâte à papier et la désinfection des édifices insalubres, a un emploi très limité; il ne s'en consomme pas même 20.000 tonnes par an en France. Dans ces conditions, une seule usine électrolytique produisant 8 à 10.000 tonnes de soude, ce qui est très peu, trouverait à peine, à elle seule, à écouler tout son chlore.

On cherche d'autres applications du chlore; un nouveau produit semble devoir en absorber beaucoup : c'est le tétra. chlorure de carbone, corps liquide contenant $92 \%$ de chlore, d'odeur agréable et possédant, comme la benzine, les essences et le sulfure de carbone, la propriété de dissoudre les corps gras, mais avec cet avantage de n'être pas inflammable et de ne pas former avec l'air de mélanges détonants. Il pourra donc remplacer les corps précédents 
dans une foule d'applications. Des essais très sérieux de fabrication se poursuivent en ce moment même de divers côtés.

- La fabrication des hypochlorites alcalins s'opère par plusieurs procédés dont le plus connu est celui de $M$. Hermite. L'électrolyte, formé d'un mélange de deux dissolutions de chlorure de sodium et de chlorure de magnésium dans de l'eau, circule continuellement de bas en haut dans un électrolyseur formé d'une cuve en fonte galvanisée; les cathodes sont formées de disques en zinc montés sur deux arbres parallèles qui tournent lentement; les anodes dont la surface active est constituée par une toile de platine, sont placées entre chaque paire de cathodes. L'intensité du courant est de ıooo à I 200 ampères sous une différence de potentiel d'environ 5 volts. Ce procédé est employé par un certain nombre de fabriques de papier.

- Lafabrication des chlorates est basée sur ce fait que lorsqu'on électrolyse à chaud et avec une concentration déterminée une solution d'un chlorure alcalin, l'hypochlorite d'abord formé, se transforme ensuite en chlorate. Le procédé Gall et Montlaur consiste à opérer cette transformation dans des appareils électrolyseurs formés de cuves rectangulaires montées en tension et très soigneusement isolées du sol. Un compartiment reçoit la cathode en fer et l'autre l'anode en platine formée d'une feuille de I/ 10 de milimètre d'épaisseur. Le chlorure de potassium, dont la solution est chauffée vers $60^{\circ}$, est décomposé par le courant; on ramène par une circulation mécanique le chlore dégagé à l'anode en contact avec la soude caustique produite à la cathode ; l'hypochlorite qui se forme d'abord se transforme peu à peu en chlorate sous l'action de la chaleur. Les appareils sont alimentés en chlorure d'une façon continue.

On sait que les chlorates sont employés à la préparation de l'oxygène, de certains produits pharmaceutiques et des allumettes. Ces derniers temps, les usines Chedde, à l'aide de procédés spéciaux et perfectionnés, préparent sous le nom de Cheddite des explosifs d'une puissance supérieure à ceux actuellement connus, d'un maniement beaucoup moins dangereux et d'une conservation plus facile.

Enfin, le conférencier dit quelques mots des nombreuses applications de l'électricité et de l'électrolyse à la chimie organique. Au nombre de celles-ci sont: la rectification des alcools, leur vieillissement artificiel, la préparation des matières colorantes, le tannage électrique, etc., etc.

Principales Industries Electrométallurgiques. - Les plus importantes sont: le raffinage électrolytique des métaux: cuivre, plomb, argent, etc., qui s'opère par la voie humide; l'extraction des mélaux : sodium, aluminium, fer, etc., qui s'obtient par la voie sèche.

Parmi ces applications $M$. Gall ne signale que les plus intéressantes. L'une d'elles est la fabrication des tubes de cuivre électrolytiques. Il aborde ensuite la préparation des métaux par voie sèche et la question de l'emploi des fours électriques pour l'obtention des métaux rares et la préparation des fontes réactives, ainsi que pour la fabrication des carbures, Le conférencier fait ressortir l'influencz remar- quable que les travaux de M. Moisson ont exercé sur ces industries. Devant publier prochainement ici des articles sur ces questions nous abrégeons et rappelons simplement les grandes lignes de la conférence de M. Gall.

Le procédé Héroult pour la fabrication de l'aluminium est basé sur l'électrolyse du fluorure double d'aluminium et de sodium à l'état de fusion igncé ; les usines de La Praz, Saint-Michel de Maurienne et Froges l'exploitent avec succès ( 1$)$. Les procédés de préparation du sodium sont nombreux, les uns, tels que les procédés Grabau, Borchers, le recueillent à l'état métallique; d'autres, comme les procédés Vautin et Hulin, le recueillent à l'état d'alliages. M. Gall termine par la description des principaux essais de réduction des minerais de fer au four électrique, au nombre desquels les plus remarquables sont ceux du capitaine Stassano en Italie, et de M. Héroult en France.

Il conciut de cette revue rapide que l'électrochimie et en particulier l'électrométallurgie, ne sont encore, en un grand nombre d'applications, qu'à leurs débuts mais que, par les difficultés déjà vaincues dans un certain nombre de ces applications, on peut espérer, à assez brève échéance, un grand développement des autres branches de cette belle industrie. La France, dit-il, est le pay's privilégié de l'électrochimie, puisqu'elle a en abondance ces forces naturelles, génératrices économiques des courants électriques puissants, sans lesquels ces applications ne peuvent prospérer. Il faudrait, pour aider au développement des diverses industries électrochimiques, que les pouvoirs compétents accordent à leurs produits des tarifs de transport et de douane leur permettant d'atteindre facilement l'exportation; sans cela des industries nées sur notre sol péricliteront faute de débouchés qu'elles pourraient trouver à l'étranger. M. Gall propose au Congrès d'émettre un vœu pour demander aux administrations compétentes qu'une assimilation libérale applique aux nouveaux produits les bases fixées pour les anciens produits chimiques (2).

\section{L'ONDOGRAPHE DE M. HOSPITALIER}

Conférence de M. Hosptalier

Tous les Congressistes ont pu voir fonctionner, dans lune des salles du Congrès, l'Ondographe inventé par M. Hospitalier, l'ingénieur-électricien bien connu.

Invité à prendre la parole, l'inventeur explique le fonctionnement de cet ingénieux appareil.

Il a pour but d'inscrire directement sur une bande de papier les courbes représentatives d'un phénomène électrique (f. e. m., d. d. p., intensité, puissance, etc.).

Au lieu de rendre l'organe mobile entièrement ljé aux variations du phénomène alternatif, comme dans les appareils de MM. Blondel et Abraham, M. Hospitalier retarde

(1) M. Gall explique ce qu'est l'Aluminothermie. On lira plus loin le compte-rendu des expériences que le conférencier a faites devant les membres du Congrès à l'usine de Prémont.

(2) Ce vou a été adopté à l'unanimité et nous avons reproduit sa rédaction dans notre précédent numéro. 
le mouvement de cet organe, mais sans en dénaturer la forme, afin de le rendre directement enregistrable sur une bande de papier.

A cet effet, le cadre mobile d'un galvanomètre, genre Deprez et d'Arsonval, est soumis, non plus à un effort continu et proportionnel à chaque instant à la grandeur du phénomène alternatif, mais à une série d'impulsions fournies, à intervalles de temps égaux, par la décharge d'un condensateur que charge, également à intervalles de temps égaux, le courant alternatif à étudier.

On conçoit facilement que si le temps qui s'écoule entre deux décharges consécutives du condensateur est légèrement différent de celui que met le phénomène alternatif à parcourir une période, la grandeur des impulsions variera lentement, mais dans le même rapport que la grandeur du phénomène à étudier. L'aiguille du galvanomètre tracera alors une onde qui aura été formée en n'utilisant ainsi qu'une petite partie seulement de chaque onde du phénomène alternatif.

La charge du condensateur et sa décharge dans l'appareil de mesure se fait par l'intermédiaire d'un commutateur cylindrique actionné par un petit moteur synchrone qu'alimente le courant à étudier. Pour que le temps entre deux charges ou décharges du condensateur soit légèrement différent de cclui d'une période du courant alternatif, tout est réglé pour que, lorsque le moteur fait inoo tours, le commutateur en fasse 999 .

Cet appareil vraiment industriel rendra de grands services à tous ceux qui utilisent et étudient les courants alternatifs.

Erratum. - Nous avons, dans notre precedent no, fait figurer parmi les vice-presidents du Congrès, M. A. Corvuaul, ingenieurélectricien à Paris, tandis que cette fonction était remplie par M. E. CORNUAULT, ingénieur à Paris, Président du syndicat de Industrie du Gaz en France.

Nous prions Messieurs Cornuavlt, PÈRE ET FrLs, tous deux membres du Congrès, d'excuser l'erreur typographique qui a fait confondre leurs titres.

\section{VISITE DES USINES}

Les installations hydro-électriques des Alpes avant déjà fait l'objet de nombreuses monographies détaillées dans les revues techniques, nous nous bornons ici à rappeler les dispositions les plus intéressantes de celles qu'a visitées le Congrès. Nous ne donnons pas non plus de vues photographiques de ces usines, ayant pensé que nous n'apprendrions rien à nos lecteurs qui les ont déjà vues dans maintes publications scientifiques. A ceux qui désireraient les connaitre avant l'apparition des volumes du Congrès, nous conseillons le magnifique ouviage de M. R. de la Brosse, ingénieur en chef des Ponts et Chaussées: L_Fs INSTALlations Hydro-Électriques dans LA RÉGION DEs Alpes (A. Gratier et J. Rey, éditeurs à Grenoble),

Nous croyons également que notre description sommaire offrira plus d'intérêt étant divisée de la manière suivante : Barrages et Prises d'eau. - Canaux de dérivation et Conduites forcées. - Equipement électro-mécanique des usines. - Utilisation de l'énergie électrique, que si nous la présentions sous la forme de monographies successives.

On voudra bien excuser l'imperfection de ce travail, auquel le temps et la place dont nous disposons ne nous ont pas permis de donner la précision méthodiqne et le développement que nous eussions désiré. Au surplus, nos descriptions d'usines sont très inégales, n'ayant pas eu sur toutes le même nombre de renseignemenls. Mais à celles qui n'ont pas ici la mention qu'elles méritent, nous ouvrirons bien volontiers nos colonnes pour réparer toutes les lacunes.

\section{E.-F. Côte.}

\section{BARRAGES ET PRISES D'EAU}

Comme le dit excellemment M. de la Brosse, dans l'ouvrage précité, "le visiteur, même ètranger aux questions de cet ordre, est tout d'abord frappé par la variété des moyens d'exécution. "Il est donc difficile d'établir une classification parmi les ouvrages qui ont été visités et d'adopter dans notre description tel ordre plutôt que tel autre. Néanmoins, pour en suivre un, nous considérerons successivement $: 1^{\circ}$ les installations utilisant de gros débits et de faibles chutes $; 2^{\circ}$ celles mettant en ceuvre des hauteurs de chutes et des débits moyens; 30 les usines où le débit dérivé est relativement faible, mais la hauteur de chute très grande (1). Il est vrai que nous aurions pu suivre l'ordre inverse.

Faibles chutes et gros débits. - Barrage d'Avignonet. - Des installations visitées, le barrage d'Avignonet (2) est celui qui doit figurer le premier dans cette classification. Le débit dérivé peut atteindre $40^{\mathrm{m}^{3}}$ et la chute totale est de 27 mètres. Etabli sur le Drac à 3o kilomètres en amont de Grenoble, au fond d'une gorge formée de rochers à pics, l'ouvrage barre le torrent sur la largeur du lit très resserré en cet endroit; sa hauteur est de 20 mètres $(27$ mètres avec les fondations); il a 24 mètres d'épaisseur à la base et $4 \mathrm{~m} .7^{5}$ au sommet. Tout entier construit en béton de ciment avec revêtements de moellons protégeant les surfaces découvertes, ce barrage audacieux réalise toutes les garanties de sécurité vollues, pour résister à l'assaut du Drac, au régime éminemment torrentiel.Pendant les grandes crues, il fonctionne en déversoir; à l'aval, le radier se profile en forme de doucine, ce qui a pour effet d'amortir la chute de l'énorme nappe déversante et d'empêcher les affouillements; en amont de l'ouvrage, un massif d'enrochements le protege de même contre les affouillements. Les galets apportés par le torrent sont évacués latéralement par un pertuis muni d'une grande vanrie de décharge de $9^{m} \times 7^{m}$. Le canal d'amenée s'ouvre à $2^{\mathrm{m}} 5 \mathrm{o}$ au-dessus du radier du pertuis.

Prise d'ean de Champ. - Suivant notre classification, nous devons maintenant considérer la prise d'eau qui alimente l'usine de Champ. Hauteur de chute : $37^{\mathrm{m}} 4^{\circ}$; débit dérivé : 17 mètres cubes (3).

(1) A propos de ces dénominations : faibles, moyennes, hautes chutes, certains pensent qu'il y aurait lieu de fixer les idées sur les chiffres dulimitant entre elles ces hauteurs de chute. M. le commandant Audebrand, en un article publié dans le no 4 de cette Revuc, a proposé un essai de classification pour répondre à ce désideratum; nous invitons nos lecteurs à s'y reporter.

(2) Appartenant à la Société Grenobloise de Force et Lumiere.

(3) Appartenant à la Societé Hydro-électrique de Fure et Morge. Nous pouvons donner une description assez complète des diverses parties de cette belle installation, grâce à l'extrême obligeance du Directeur général, M. Lépine, ingénieur des Arts et Manufactures, auteur de ce projer qui lui fait grand honneur. 
Etablie en aval du pont de la Rivoire situé sous la ligne du chemin de fer de Grenoble à Gap, par Veynes, elle se compose de deux barrages, l'un fixe, l'autre mobile, d'un canal de dérivation et d'une chambre d'eau, point de départ de la canalisation forcée aboutissant à l'usine. Le barrage fixe est en béton, c'est un massif d'une longueur de 130 mètres, d'une largeur de I 2 mètres et d'une épaisseur variant de 2 à 5 mètres, suivant le profil transversal du Drac.

Ce massif de bíton est recouvert d'un pavage rudimentaire en gros enrochements pour empêcher les graviers charriés par le torrent de désagréger le béton. C'est d'ailleurs un simple barrage de prise, n'ayant qu'une hauteur de $I^{\text {mb5o à } 2}$ mètres au-dessus du lit du torrent: il réunit la rive gauche du Drac à la pile fondée en riviere qui sert d'union entre le barrage fixe et le barrage mobile.

Le barrage mobile qui a 16 mètres d'ouverture, est composé de deux vannes de 8 mètres de longueur et 1 m5o de hauteur. Ces vannes sont manouvrées d'une passerelle mítallique prenant appui d'une part sur la pile, d'autre part sur le couronnement du mur du bajoyer. Une aiguille métallique sert de support intermédiaire à la passerelle et de point d appui aux glissières des vannes. Ce barrage mobile a pour but de maintenir le courant le long du bajoyer atin d'éviter le dépôt du gravier devant les grilles. Le l raccharrie en eftet des quantités considérables de gravier, et il faut prendre des dispositions toutes spéciales pour éviter qu'il ne se dípose devant les grilles et n'entre dans le canal de dérivation.

Le mur de bajoyer est en béton de ciment; il a une longueur de 80 mètres et est percé de 15 ouvertures de 2 mètres de largeur sur $1^{\text {tu}} 50$ de hauteur qui peuvent être fermées par des vannes. Une grille de forme spéciale est installée tout le long de ce mur, du côté du Drac; elle est composée de lames de fer disposées en persiennes, de facon que les cailloux, cheminant le long de ces lanes et entrainés par le courant produit par l'ouverture du barrage mobile, ne puissent pénétrer dans le canal de dérivation.

Le mur de bajoyer est fondé sur pilotis, le barrage mobile repose sur un caisson descendu à 9 mètres de profondeur au moyen de l'air comprimé.

Le canal de dérivation, qui reçoit l'eau qui a passé par les ouvertures du bajoyer, a une longueur de 600 mètres et une largeur variant de 60 à 22 mètres, avec une profondeur allant en croissant depuis I $^{\mathrm{m} 50}$ jusqu’à 4 mètres. Ce canal est exécuté pa:tie en déhlai, partie en remblai; ses parois latérales sont garnies d'un revètement de chaux hydraulique; la pente du fond du canal est de $4 \mathrm{~m} / \mathrm{m}$ par mètre.

La chambre d'eau qui sert de point terminus au canal de dérivation, et de point de départ à la canalisation sous pression, est en béton de ciment; elle a une surface totale de goo $\mathrm{m}^{2}$ et une profondeur moyenne de 4 mètres. Cette chambre est divisée en deux compartiments : le premier, qui reçoit directement l'eau du canal, est destiné à retenir les sables entraînés par les eaux. Ces sables sont évacués au moyen de rigoles convenablement aménagées dars la sole de la chambre et aboutissant à six vannes de chasse placées dans le mur gauche de la chambre d'eau, mur disposé en forme de déversoir. Un canal de vidange rejette au Drac ces sables cn même temps que les eaux qui passent dans le déversoir. Un mur de décantation, surmonté d'une grille à feuilles, sépare le premier compartiment du second, auquel vient se souder le tuyau en béton armé formant la première partie de la canalisation sous pression. Une vanne de $3 \mathrm{~m} 50$ sur 4 mètres permet de fermer à volonté l'entrée du tuyau. La lame d'eau surmontant la génératrice supérieure de l'intrados du tuyau a une épaisseur de $2^{m}$ po, de façon à éviter les rentrées d'air. L'entrée du tuyau est d'ailleurs évasée en forme de trompe pour faciliter l'introduction régulière de l'eau.

Afin d'assurer une complète sécurıté de marche aux usines desservies par cette installation, l'usine de Champ a été équipée pour utiliser le débit minimum du Drac.

Barrage et prise d'ean de Servoz. (Chemin de fer électrique $P$-L.-M. du Fayet à Chamonix). - Ici, la hauteur de chute est de 40 mètres et le débit utilisé de 8 mètres cubes. L'usine de Servoz est alimentée par la première partie de la chute que la Société des Forces Motrices de l'Arve a aménagée pour son usine de Chedde, dont le canal d'amenée est alors directement raccordé au canal de fuite de l'usine de Servoz. En été, les $12^{\mathrm{m}^{3}}$ de débit maximum peuvent donner une puissance de 4560 HP sur l'arbre des turbines et, en hiver, cette puissance est encore de $2280 \mathrm{HP}$, alors que 1420 che. vaux seulement sont réservés pour le chemin de fer.

Le barrage placé sur l'Arve comprend deux parties séparées par un îlot de rochers. Sur le bras principal se trouve un double barrage à aiguilles en bois et sur le bras secondaire, un barrage à poutrelles formant déversoir. L'eau, après avoir traversé la grille de la prise d'eau, faite directement dans la berge rive gauche, pénètre dans une chambre souterraine de décantation, longue de 230 mètres où, pour un débit de $8^{\text {in } 3}$, la vitesse n'est que de $0^{\mathrm{m}} 47$ par seconde. Les dépôts de sable, ainsi bien facilités, sont ensuite évacués par des chasses énergiques.

Hauteurs de chute et débits moyens. - Prise d'ean de l'usine des Clavaux. - Située sur la Romanche, près de la route du Bourg d'Oisans, à 3o kilomètres de Grenoble, elle alimente l'usine de la Compagnie Universelle d'A célylène, créée en '1897-98, par M. L. Hulin. La hauteur de chute est de 42 mètres; le maximum ( 15 mètres cubes) du débit dérivé est atteint pendant 8 mois de l'année et, pendant l'hiver, il descend à 7 ou 8 metres cubes.

Cette prise d'eau est formée d'un avant-canal de dérivation directe, large de 10 mètres, lóng de 80 mètres, dont le mur forme déversoir continu du côté de la Romanche, de façon à rendre les crues inoflensives. Le barrage n'est qu'un simple seuil en blocs de pierre et ciment, formant une rectification du lit de la Romanche. La retenue ainsi formée est à la cote $47^{8}$.

Deux vannes de chasee latérale, placées en aval du canal d'amenée, permettent d'évacuer les cailloux que le courant. charrie durant la période des hautes eaux. A la suite de cet avant-canal sont installées cinq vannes d'arrêt placées de front et commandant l'accès de l'eau dans un vaste bas. sin à fond incliné où la vitesse du courant est très réduite. ce qui permet aux sables de se déposer; des vannes de purge permettent dé chasser les dépôts. Ce bassin est terminé par une grille de 25 mètres carrés destinée à arrêter les bois et les fecilles et est raccordé à la canalisation métallique par un mur de 3 mètres d'épaisseur qui enveloppe la conduite forcée.

Barrage et prise d'eau de Livet (I). - M. Drouhin qui, dans sa conférence, a si bicn établi les conditions de captage d'un torrent, était qualifié pour cet enseignement; les ouvrages qu'il a exécutés ici de main de maître, dénotent chez lui la plus entière possession de la science de l'ingénieur appliquée à ce nouvel art de l'aménagement des forces hydrauliques.

Il s'agissait, en barrant la Romanche à Livet, d'échapper à l'inconvénient des apports continus du torrent qui encombrent rapidement les bassins de retenue et de construire un ouvrage capable en tout temps, même en cas d'avarie, d'assurer l'alimentation de la prise d'eau.

(1) Société Electrochimique de La Romanche. - Hauteur de chute $=00 \mathrm{~m}$., débit dérivé maximum $=25 \mathrm{~m}^{3}$. 
M. Drouhin a adapté à ces conditions des dispositifs simples et d'une parfaite sécurité. Le barrage, de faible hauteur, ne crée que la retenue suffisante pour opérer la décantation et la chasse des apports. L'ouvrage est disposé pour offrir le maximum de résistance à la violence des eaux; c'est une voûte à axe vertical de 60 mètres de rayon sur 30 mètres de développement et dont les culées sont formées, d'un côté par un massif d'ancrage dans la berge, et de l'autre par le bajoyer du pertuis de chasse; elle est elle-même composée de trois arceaux formant voussoirs appuyés sur des piliers de $10 \mathrm{~m} \times 3 \mathrm{~m}$. Si cet ouvrage venait à céder, ce ne pourrait être, en tous cas, que sur l'un de ces arceaux et la prise d'eau fonctionnerait quand même.

Cette dernière se fait en déversoir, latéralement et immédiatement en avant d'un pertuis de chasse. Le déversoir de prise dont la crête est à ombo au-dessous de celle du barrage peut débiter $25^{\mathrm{m} 3}$ quand le niveau de l'eau retenue affleure celui-ci.

Au-dessus de ce cube, le barrage fonctionne en déversoir. Son profil transversal se dessine suivant une parabole à la partie supérieure, raccordée tangentiellement à une contrecourbe elle-même tangente au lit du torrent à l'aval; cette disposition pare très bien aux affouillements.

La prise n'admet ainsi que des eaux de surface déjà partiellement décantées; une grille inclinée à $30^{\circ}$ arrête les corps flottants.

Le pertuis de chasse, de 6 mètres de large, est commandé par trois vannes au niveau du barrage; son radier, d'une pente de $\mathrm{o}_{\mathrm{O}} 3$ par mètre, est situé à un niveau inférieur à celui du lit en amont du barrage. En soulevant un peu les vannes, on crée une chasse extrêmement énergique des apports décantés.

Telles sont les caractéristiques de cette prise d'eau qui, depuis trois ans, a assuré l'alimentation du canal de dérivation sans la moindre interruption de service, quel qu'ait été l'état de la Romanche.

Barrage et prise d'ean de "La Volta 》. - (Chute de $7^{5}$ mètres; débit dérivé de $\mathrm{I} 7 \mathrm{~m}^{3}$ ) Situé sur l'Isère, à 7 kilomètres environ en amont de Moûtiers. Il est formé de deux grandes vannes de ro mètres de largeur séparées par une pile en maçonnerie, et crée une retenue de $2^{\mathrm{m}} 50$ de hauteur. Un déversoir longitudinal de 44 mètres de longueur est disposé latéralement. Les vannes sont du systeme "Stoney " à contre-poids et roulement sur trains de galets; un seul homme peut les manœuvrer sans effort.

Quatre ouvertures de 5 mètres de largeur chacune, disposées à l'amont du barrage et normalement à son axe, laissent pénétrer l'eau dans une chambre de décantation de $80^{\mathrm{m}}$ de longueur sur $8^{\mathrm{m}}$ de largeur.

Barrage des Pyes (Usine de la Praz). - Cette usine est alimentée par deux chutes sur l'Arc, l'une de 35 mètres et l'autre de 78 mètres, avec un débit total de 12 mètres cubes en eau moyenne. La prise d'eau principale est obtenue au moyen d'un barrage formé de vannes mobiles, qui comprennent : $1^{\circ}$ une vanne principale de 14 mètres d'ouverture sur 3 mètres de hauteur, ayant un levage de 4 mètres et dont la poussée est reçue par des galets sur un chemin de roulement, qui rend sa manœuvre possible à un homme seul; son tablier se compose de panneaux de 5 centimètres d'épaisseur, en mélèze, assemblés par dix fermes, en forme de poisson, de fer en $\mathrm{U}$; elle se trouve placée entre la berge gauche et un rocher au milieu du torrent; $2^{\circ}$ trois vannes de $2^{m 5} 5$ de large placées entre ce rocher et l'ouvrage de la grille de prise d'eau; ces dernières servent au déblaiement des apports devant la grille.

Celle-ci, située latéralement, précède immédiatement le canal d'amenée et, en avant et en arrière, on a ménagé des canaux de chasse que l'on peut faire fonctionner, en ouvrant des vannes de purge disposées à cet effet, en amont et en aval de l'ouvrage de prise d'eau; l'un de ces canaux sert de trop-plein et à l'évacuation des feuilles et des corps flottants. Un bâtiment surmonte le plancher de mancuvre des vannes de grilles, de chasse et de communication entre ces divers canaux.

Hautes chutes et faibles débits. - Prise d'ean des Usines de chedde. - Les ouvrages de cette chute aménagée sur l'Arve, entre Servoz et Chedde, permettent de dériver ${ }_{1} 2^{m^{3}}$ par seconde.

La hauteur totale de chute est de 180 mètres, divisée en deux parties: la partie supérieure, de 40 mètres, est utilisée par l'usine de Servoz (chemin de fer électrique du Fayet à Chamonix); la partie inférieure, de 140 mètres, est la propriété de la Sociétédes Forces Motrices et l/sines de l'Arve. L'aménagement de cette chute se présentait dans des conditions topographiques exceptionnellement favorables. Entre Servoz, où est la prise d'eau et Chedde, oúl'eau est rendue à la rivière, l'Arve forme une boucle assez prononcée; le tracé de la chute suit à peu près exactement la corde de cette boucle. A partir de la prise d'eau de Servoz, le canal de dérivation traverse, en tunnel, un massif rocheux assez élevé (massif des Gures), puis débouche dans la chambre d'eau de l'usine hydro-électrique de la Compagnie P.-L.-M., c'est-à-dire à 40 mètres au-dessus du fond du ravin du Châtelard. Par un puits incliné qui suffit au débit total et, en partie, par les tuyaux et turbines de la précédente usine, l'eau gagne un canal de fuite, traverse le fond du ravin par un prolongement de ce canal passant sous la voie ferrée; de là, elle s'engage à nouveau en tunnel, dans la même direction que le premier, sur une longueur de 672 mètres sous la montagne des Egrats et elle aboutit sur le flanc de celle-ci dans la chambre d'eau de l'usine de Chedde, à I 40 mètres au-dessus du sol de cette usine.

Le Bassin de Lancey. - Après avoir aménagé d'abord une chute de 200 mètres sur le ruisseau de la Combe, puis une autre de 500 mètres, $M$. Bergès a ensuite réuni par un siphon, les deux cuvettes très inégales du lac Crozet, ce qui Ini a donné l'emploi d'une tranche d'eau augmentant déjà beaucoup la puissance qu'il utilisait a Lancey. Puis il a successivement entrepris de relever le niveau de ce lac, en surmontant d'un barrage le seuil rocheux qui lui sert de déversoir; d'aménager une chute de 485 mètres, en captant le ruisseau de St Mury qui coule dans une vallée proche de Lancey et dont les eaux sont issues du lac Blanc, a 2160 mètres d'altitude; enfin, de percer en plein rocher, un tunnel de 230 mètres allant chercher l'eau d'une nappe rete. nue en contre bas du seuil du lac Crozet et jusqu'alors sans emploi. 
On sait qu'en outre $M$. Bergès a formé le projet d'utiliser des lacs situés dans les parties supérieures du bassin de Lancey, de manière à aménager les 10000 chevaux que l'eau tombée sur ce bassin de 44 kilomètres carrés peut fournir sous une chute totale réalisable de 1700 mètres. Il est hors de doute que ce projet qui est bien le digne couronnement de l'œuvre du père ne soit mené à bonne fin par les fils, ses continuateurs.

Chnte de Chapareillan ( $\mathrm{r}$ ). - Utilisant le Cernon sur une hauteur de 620 mètres, elle détenait jusqu'à cette année le record de la hauteur, s'il est permis de parler ainsi. Mais elle est maintenant dépassée de 300 mètres par la chute de Vouvry, dont il sera fait mention plus loin. Le ruisseau qui alimente l'usine n'a qu'un débit d'étiage de 50 litres; la prise d'eau est simplement constituée par un barrage en maçonnerie de faible hauteur établi dans le fond du torrent et duquel part la canalisation forcée qui aboutit à l'usine en suivant les pentes du Mont Granier.

\section{GANAUX DE DERIVATION ET GONDUITES FORGEES}

10 Faibles chutes et gros débits. - Le canal de la Société Grenobloise de Force et Lumière, à Avignonet, est percé en souterrain dans le rocher, sur toute sa longueur qui est de 840 metres. Sa section est celle d'un tunnel de 4 mètres de large sur $4^{\mathrm{m}} 50$ de hauteur, y compris la voûte en plein cintre; sa pente est de 1 millimètre par mètre et il peut éventuellement débiter 40 mètres cubes à la secunde. Ses parois sont revêtues d'une chappe en ciment. A l'intérieur de la galerie, existe un déversoir régulateur de 65 mètres de longueur qui rejette au Drac le trop plein du débit. Ce canal se termine à l'aval par une chambre d'eau, d'ou partent trois conduites forcées en tôle de $2^{\mathrm{m}_{2}} \mathrm{O}$ de diamètre et de 7 à $8 \mathrm{~m} / \mathrm{m}$ d'épaisseur, longues de 79 mètres, donnant une chute franche de 23 mètres. Elles ont été construites par MM. Joya père et fils, de Grenoble, ainsi que celle de l'installation suivante.

La canalisation qui réunit la chambre d'eau à l'usine génératrice de Champ (Société Hydro-électrique de Fure et Morge) est formée d'un tyau de $3 \mathrm{~m} 30$ de diamètre intérieur et de 4600 mètres de longueur, dont 2 100 en béton armé et 2500 en tôle d'acier doux.

La partie en béton armé est destinée à supporter les pressions jus. qu'à 20 métres de bauteur d'eau; c'est un tuyau dont l'épaisseurvarie de om 20 à om 25 ; le treillis composant l'armature métallique est formé

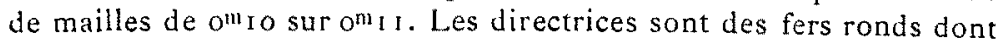
le diamètre varie de I I à $25,5 \mathrm{~m} / \mathrm{m}$; les génératrices sont également des fers ronds dont le diamètre varie de 6 à $\mathrm{i} 2 \mathrm{~m} / \mathrm{m}$. Le tuyau repose sur un berceau en béton de ombo d'épaisseur moyenne reposant luimême sur le gravier de telle façon que le tuyau est enterré de 2 mètres.

Le tuyau de tôle qui fait suite au tuyau de ciment armé est composé de feuilles d'acier doux, dont l'épaisseur varie de 7 à $15 \mathrm{~m} / \mathrm{m}$; ces feuilles sont assemblées au moyen de rivets. Le tuyau repose sur un berceau en béton de om5o d'épaisseur moyenne, enterré dans le sol.

(1) A la Société des Forces Motrices du Haut Grésivaudan possédant d'autres installations notamment celle de Pontcharra-sur-Bré.'a, utilisant une chute de 45 mètres.
Nous croyons que c'est la première fois qu'a été employée cette méthode de faire reposer une conduite métallique de grand diamètre sur un berceau en béton enterré dans le sol. Cette disposition présente cependant le très grand avantage d'empècher la déformation du tuyau pendant l'opération très délicate du remplissage et a donné d'excellents résultats. De plus, les conduites étant recouvertes d'une couche de terre et de gravier de $0^{m} 40$, les effets de dilatation ne sont plus à redouter.

La masse d'eau en mouvement dans toute la longueur du tuyau étant de $40000 \mathrm{~m}^{3}$, se déplaçant avec un vitesse de 2 mètres à la seconde, il était nécessaire de prévoir des dispositions spéciales pour éviter les coups de bélier, en cas d'arrêt brusque de l'usine. On a, à cet effet, installé sur le tuyau trois cheminées, dont deux en béton armé et une en tôle. Les cheminées en béton armé ont respectivement des hauteurs de 9 et 20 mètres. Celle de 9 mètres est située à 400 mètres de la chambre d'eau; celle de 20 mètres est située au point d'union de la conduite en tôle et de la conduite en béton armé. Elle a ${ }_{1}^{\mathrm{m}}$ ro de diamètre intérieur et porte autour de son soubasse. ment un réservoir, également en béton armé, destiné à recevoir l'eau qui pourrat s'écouler le long de ladite cheminée.

La cheminée en tôle, de 35 mètres de hauteur, $3 \mathrm{~m} 30$ de diamètre à la base et $\mathrm{I}^{\mathrm{m}} 4 \mathrm{O}$ au sommet, est installée à l'extrémité du collecteur, dont elle est en réalité le prolongement; elle est reliée avec lui au moyen d'un coude arrondi et sa partie supérieure est à la cote 300,60 . Cette cheminée est surmontée d'un réservoir également en tòle d'acier de 3 mètres de diamètre, 2 mètres de hauteur, duquel partent trois tuyaux de om6o de diamètre qui descendent à l'extérieur de la cheminée pour aboutir au canal de fuite. Ces tuyaux sont destinés à écouler l'eau qui pourrait monter dans le réservoir à la suite des coups de bélier.

Enfin, des vannes compensatrices et un amortisseur d'un type spécial empêchent la production de surpressions dans la canalisation ou tout au moins en diminuent l'importance.

La hauteur théorique de la chute utilisée est de $37^{\mathrm{m}} 40$ mesurée entre le niveau de l'eau dans la chamhre d'eau et le niveau supérieur de l'eau, dans le canal de fuite.

Dans l'installation de la Compagnie P.-L.-M .. à Servoz, l'eau en sortant de la chambre de décantation franchit un déversoir et, comme nous l'avons déjà dit, arrive à la chambre de mise en charge par une galerie souterraine, longue de 550 mètres, pouvant débiter $12^{\mathrm{m}^{3}}$ par seconde à la vitesse de $2^{\mathrm{m}} 4 \mathrm{o}$.

Quatre conduites forcées, en tôle d'acier extra doux et de $\mathrm{o}^{\mathrm{m}} 95$ de diamètre intérieur, conduisent l'eau aux turbines. 11 n'y a pas de joints de dilatation et l'on ne compte à cet égard que sur les coudes des conduites.

$2^{\circ}$ Chutes et débits moyens. - Sur la Romanche, aux usines des Clavaux, la canalisation forcée qui fait immédiatement suite au grand bassin de décantation de la prise d'eau, est formée d'une conduite cylindrique en tôle d'acier doux, ayant un diamètre intérieur de $2^{\mathrm{m}} 50$ et une longueur d'environ 1100 mètres; son épaisseur est de $6,5 \mathrm{~m} / \mathrm{m}$ à l'origine et $13 \mathrm{~m} / \mathrm{m}$ au collecteur, près des turbines.

Cette conduite repose sur des piliers en maçonnerie, couronnés d'une tôle plate, par l'intermédiaire de semelles métalliques librement posées. Les effets de dilatation trouvent une compensation dans les nombreux coudes que présente la conduite sur son parcours.

La hauteur de chute est de 42 mètres, dont 35 mètres par pression effective et 7 mètres par aspiration à la sortie des turbines. La retenue à la prise est à la cote 478 et le niveau de l'eau dans le canal de fuite à la cote 436 .

- A Livet (Société Electro-chimique de la Romanche), nous retrouvons dans les dispositions adoptées pourle canal 
de dérivation et la conduite forcée, la même ingéniosité et le même souci de la sécurité que nous avons vus dans l'établissement des ouvrages de la prise d'eau.

Le canal d'amenée s'amorce à la suite d'une chambre de décantation placée immédiatement après le déversoir de prise. Ce canal est en souterrain sur 2000 mètres, c'est-àdire sur toute sa longueur; sa section de ${ }_{1} \mathrm{~m}^{2} \mathrm{est}$ de forme circulaire d'un diamètre de $3 \mathrm{~m} 75$. Il est entièrement revêtu d'une paroi en béton de ciment, dont l'épaisseur varie de $\mathrm{O}^{\mathrm{m}_{2} 5}$ sur le rocher à $\mathrm{I}$ dans les terrains meubles. Sa pente est de 15 à $10 \mathrm{~m} / \mathrm{m}$ par mètre, donnant une vitesse de 2 mètres à la seconde. Trois décantations successives des sables s'opèrent sur le parcours à l'aide de petits barrages obliques et les chasses se font dans les anciennes lunettes d'attaques.

La chambre d'eau oú ce canal aboutit, entièrement creusée dans le rocher à 60 mètres au-dessus du sol de l'usine, est divisée en decx parties : une chambre de décantation et une chambre de travail. Le batardeau qui les sépare est surmonté de trois vannes verticales pouvant les isoler complètement; l'eau pénètre de la première dans la seconde par déversement sur la cloison de séparation; les sables décantés dans la première sont évacuès par deux vannes de chasse et l'un de ses murs forme déversoir qui rejette à la Romanche le trop plein de la dérivation. C'est du sol de la deuxième chambre que part la conduite forcée. Celle-ci est placée dans un puits vertical, puis dans une galerie horizontale sous le sol de l'usine; son diamètre est de $2^{\mathrm{m}} 5 \mathrm{o}$, l'épaisseur de la tôle varie de 5 à $15 \mathrm{~m} / \mathrm{m}$. L'espace entre la paroi du puits vertical et le tuyau a été bourré en béton de ciment.

Si l'on se reporte à la conférence de M. Drouhin, on constatera qu'il a lui-même rigoureusement appliqué ici les formules des meilleures conditions d'établissement d'une dérivation, au double point de vue de la sécurité des ouvrages et de la régularité du fonctionnement de l'installation.

- L'installation de la Société La Volta, à Moûtiers, comporte une dérivation dont la longueur totale est de 3280 mètres, dont 3080 en tunnel et 200 à ciel ouvert. La pente du canal est de $2 \mathrm{~m} / \mathrm{m}$ par mètre dans les parties non revêtues de maçonnerie et de $\mathrm{I}, 5 \mathrm{~m} / \mathrm{m}$ dans les parties recouvertes d'un béton lissé. Ce canal aboutità une chambre de mise en charge de 60 mètres de long divisée en trois sas longitudinaux: le premier sert à la décantation des sables que l'on chasse par une vanne de purge; le second, recouvert de tôles perforées pour arrêter les corps flottants, ne reçoit que l'eau de surface et la conduit dans la chambre de départ des conduites forcées; le troisième taillé dans le rocher recueille les eaux du déversoir superficiel ainsi que les purges du premier sas.

Trois conduites métalliques ont été prévues, mais une seule est actuellement en place; ellc a ${ }^{\mathrm{m}}{ }^{\mathrm{m}} 90$ de diamètre et est formée de tôles d'acier de 6 à $10 \mathrm{~m} / \mathrm{m}$ d'épaisseur.

Tous ces ouvrages de dérivation, depuis le barrage et la prise d'eau ontété traités avec beaucoup de méthode, d'ingéniosité et de soin; aussi cette installation a-t-elle à juste titre fait l'admiration des congressistes. S'il est vrai qu'un mécompte s'est produit au début de la mise en eau du canal, les causes quiul'ont produit ont été vites supprimées, et l'on peut aujourd'bui proposer en exemple à tous les points de vues, cette dérivation remarquablement bien conçue et exécutée.

L'Usine de La Praz est alimentée par deux conduites en tôle d'acier ayant : la première 2 mètres de diamètre, I 200 mètres de long et fournissant une chute de 35 mètres; la seconde $2^{m_{4}}$ de diamètre, 1000 mètres de long et faisant face à une charge de $7^{2}$ mètres.

Cette dernière est la plus intéressante à étudier. Elle a été construite sur les plans de M. Héroult, avec des tôles provenant des Aciéries de Saint-Etienne et dont l'épaisseur varie de $5 \mathrm{~m} / \mathrm{m}$ à la prise, à $15 \mathrm{~m} / \mathrm{m}$ au collecreur; le poids total du métal employé est de 785 tonnes. La vitesse de l'eau y est de $2^{\mathrm{m}} 75$ environ, à la seconde. Elle ne comporte pas de joints de dilatation, les courbes qu'elle présente suffisant à en compenser les effets. Sur la plus grande partie de sa longueur, elle repose simplement sur le sol ou dans des tranchées de faible profondeur; seuls, quelques piliers de maçonnerie la soutiennent aux coudes.

La partie la plus remarquable de cette canalisation est l'arc tubulaire de 50 mètres de portée qui, près de l'usine, franchit la rivière sans aucun support intermédiaire. Cet arc, de même section que la conduite, est, à ses extrémités, solidement encastré, au moyen de cornières, dans des culées en maçonnerie armée. Ainsi, sans interposition de consoles, d'une manière très simple et avec toute la sécurité désirable, on a pu empêcher le dévers et le déplacement des naissances. On voit que cette solution est d'une remarquable élégance et d'une belle hardiesse, si l'on songe que la conduite transmet normalement une puissance de roooo chevaux.

Le calcul des efforts supportés par le métal indique que les forces élastiques maxima sont développées, non pas lorsque l'arc est en charge, mais quand il est simplement rempli. Les efforts dus au régime normal de charge, diminués par la pression intérieure, ne dépassent pas $4^{\mathrm{k}} 750$ par $\mathrm{m} / \mathrm{m}^{2}$ de section brute, soit $6 \mathrm{k} 350$ par $\mathrm{m}_{1}^{2} \mathrm{~m}^{2} \mathrm{de}$ section nette. Sur les culées, la poussée horizontale équivaut à 30 r tonnes et la réaction verticale à 142 tonnes. Pour construire cet arc tubulaire, on a successivement rivé, à partir de chaque culée, les tronçons amenés par une passerelle de service, et de manière à ce que les deux avant-becs en porte-à-faux. ainsi formés symétriquement, vinssent se rejoindre à la clef de voùte.

30 Hautes ehutes et faibles lébits. - La dérivation de la Société des Forces motrices et Usines de l'Arve, à Chedde, à partir du canal de fuite de l'usine hydro-électrique du P.-L.-M, à Servoz, comprend d'abord le tunnel, dit galerie des Egrats, ayant une section de $2^{\mathrm{m}} 50$ de large sur $2^{\mathrm{m}_{2}} 5$ de hauteur, longue de $67_{2}$ mètres. Au débouché de cette galerie, l'eau est à volonté adimise dans les tuyaux en tôle de l'usine ou renvoyée directement à l'Arve par un puits incliné qui débouche au-dessous de la route de Chamonix, et qui sert ainsi de déversoir à la galerie. De l'extrémité aval du tunnel, l'eau est amenée à l'usine par deux tuyaux métalliques de ${ }^{\mathrm{m}}{ }^{4}$ o de diamètres. La tôle d'acier dont ils 
sont formés a une épaisseur de $6 \mathrm{~m} / \mathrm{m}$ au sommet et de $14^{\mathrm{m} m}$ à la base.

Un tuyau dessert la partie nord de l'usine et l'autre la partie sud et chacun d'eux porte six prises d'alimentation des turbines. Sur un gros embranchement, en boucle, reliant ces tuyaux par en dessus et en face de la partie centrale de l'usine, on a disposé des prises d'cau pour tous les services généraux et pour ceux communs aux fabrications. dans les parties sud et nord de l'usine, de façon que ces services soient assurés par les deux canalisations ou, en cas d'arrêt de l'une d'elles, par l'une ou l'autre seulement. Toute la partie métallique de cette installation a été exécutée par MM. Joya.

A l'Usine de Chapareillan, la conduite forcée construite par les ateliers Joya, de Grenoble, a une longueur de 3650 mètres, son diamètre est de om3o et l'épaisseur de ses tôles varie de 3 à 14 millimètres.

Les travaux d'aménagement du Bassin de Lancey ne comprennent pas moins de cinq grandes canalisations forcées en tôle, également construites par MM. Joya.

La plus ancienne, installée en 1873 , a un diamètre de om3o, une longueur de i6on mètres et des épaisseurs de tôle variant de 4 à $10 \mathrm{~m} / \mathrm{m}$; elle rachète une différence de niveau de 200 metres.

La seconde, dutant de $188 \mathrm{r}$, a un diamètre de $\mathrm{o}_{40}$, une longueur de 250 mètres et fournit une chute de 220 mètres; ses tôles sont uniformément épaisses de $9^{\mathrm{m}} / \mathrm{m}$.

En 1882 , M. Bergès faisait installer une troisième conduite de om'3o de diamètre, de 2500 mètres de longueur, avec des tôles de 4 à $16 \mathrm{~m} / \mathrm{m}$, créant une chute de 500 mètres.

Il captait. en 1885 , une deuxième chute de 500 mètres avec une conduitede 3200 mètres de développement sur om 40 de diamètre avec des épaisseurs de tôle allant de 7 à $16 \mathrm{~m} / \mathrm{m}$.

Enfin, en 1893 , M. Bergès installait encore une canalisation forcée rachetant une différence de niveau de 500 mètres, d'un développement de I 800 mètres avec $0^{m} 45$ de diamètre et des tôles de 3 à $16 \mathrm{~m} / \mathrm{m}$.

Ces zanalisations, qui aménagent une puissance hydraulique d'environ 5000 chevaux, ont employé au total un peu plus de 1000 tonnes de tôle.

\section{ÉQUIPEMENT ÉLECTRO-MÉCANIQUE DES USINES EMPLOI DE L'ENERGIE}

Nous avons cru qu'il convenait ici de changer lordre de notre classification, afin de faciliter les comparaisons par le rapprochement les unes des autres: $x^{0}$ des usines qui font du transport de force; $2^{\circ}$ de celles qui font de la traction électrique; 30 de celles qui font de lèlectrochimie.

Installations de transport de force. - Deux usines, celle de la Société hydro-électrique de Fure et Morge et celle de la Société grenobloise de Force et Lumière, utilisent, entre Champ et Moirans, soit sur un parcours de 35 kilomètres, la même ligne de transport électrique de l'énergie, laquelle a été construite par la Société Franco-Suissé pour l'Industrie Electrique, et installée sous le nom de ia Société G. de F. et L. C'est, jusqu'ici, le seul exemple, en Europe, d'un transport de force commun à deux exploitations de ce genre. Examinons-en successivement les diverses particularités.

Usine de Champ. - Elle contient actuellement cinq groupes de turbines-alternateurs de $1350 \mathrm{HP}$ chacun, deux groupes de turbines-excitatrices de 50 HP chacun et une petite turbine de 5 槚 destinée à alimenter les deux accumulateurs hydrauliques nécessaires à la mancuvre des régulateurs à servo-moteurs.

Les turbines, fournies par la maison Neyret-Brenier de Grenoble, sont du type centripète avec distributeur à tiroir cylindrıque; clles fonctionnent avec une aspiration de $5 \mathrm{~m}$. Elles sont reliées aux alternateurs par des manchons munis de taquets en caoutchouc travaillant à l'écrasement. Leur vitesse est de 300 tours à la minute, la quantité d'eau employée à pleine charge est d'environ $4^{\mathrm{m} 3}$ à la seconde. Trois des turbines sont munies de régulateurs antomatiques à servo-moteurs et accouplés à des vannes compensatrices dont la disposition est telle que lorsque le régulateur ferme le distributeur de la turbine, il ouvre d'une quantité équivalente les orifices de la vanne compensatrice, de sorte que la quantité d'eau employée par une turbine à régulateur est théoriquement constante, les $4^{\mathrm{In}^{3}}$ nécessaires à son fonctionnement à pleine charge passant soit par les orifices du distributeur, soit par ceux de la vanne com. pensatrice. La vitesse de l'cau dans le tuyau est done sensiblement régulière et la production de coups de bélier à peu près supprimée.

I.e matériel électrique de l'usine gènératrice a été entièrement fourni par la maison Brown-Boveri et Cie de Baden.

Il comprend cinq alternateurs, deux dynamos d'excitation et cinq transformateurs-élévateurs.

Les alternateurs triphasés sont à arbre horizontal et inducteur tournant. Chacun d'eux peut absorber sur l'arbre $1350 \mathrm{HP}$, en travaillant sur des circuits dont cos. $\varphi=\mathbf{0 , 8}$.

Iis fournissent du courant alternatif triphasé avec une tension composée de 3000 volts; la fréquence du courant est de 50 périodes par seconde; leur vitesse étant de 300 tours par minute, le nombre des pôles de l'inducteur tournant est de 20 .

Les dynamos à courant continu, destinées à fonctionner comme excitatrices, sont au nombre de deux; chacune d'elles peut absorber sur son arbre $150 \mathrm{HP}$; l'induit tourne avec une vitesse de 500 tours à la minute; elles sont excitées en dérivation et la tension du courant produit est de r 10 volts.

Il y a cinq transformateurs élévateurs. La puissance de chacun d'eux est de ri5o KW; les trois noyaux sont disposés dans le même plan, les bobints primaires à 3000 volts cont subdivisées, sur chaque phase, en trois parties égales mises en série de façon à permettre, par un changement des connexions dans ces bobines, d'utiliser la tension de 1000 volts en groupant les trois parties en parallèle. Les extrémités des bobines secondaires sont rattachées à six bornes situées sur la caisse du transformateur de telle manière que l'on peut, par une mancuvre simple, faite au tableau de haute tension, connecter ces bobines soit en triangle, pour abtenir la tension de 15000 volts, soit en étoile pour obtenir la tension de 26000 volts.

Les noyaux et les bobines sont plongés dans un bain d'huile refroidie par une circulation d'eau dans un double serpentin baignarit dans l'huile.

Les tableaux sont au nombre de trois:

10 Tableau à moyenne tension, situé au rez-de-chaussée, entre la salle des machines et la salle des transtormateurs:

$2^{0}$ Tableau à basse tension, situé au premier étage, sur la passerelle de service;

3o Tableau à haute tension, situé au milieu de la salle des tableaux au premier étage.

Ces tableaux renferment tous les appareils de mesure, de protection et de sécurité nécessaires pour assurer la bonne marche de l'usine; ils ont été construits avec les derniers perfectionnements et sont remarquables par leur simplicité.

Usine d'Avignonet. - Elle disposera bientôt de six unités de 1800 chevaux comprenant des turbines Picard et Pictet, 
à libre déviation, accouplées à des alternateurs Schneider de $1750 \mathrm{~K} . \mathrm{v}$. amp., et donnant du courant triphasé directement, c'est-à-dire sans aucune transformation, à la tension de 26000 volts. C'est, à notre connaissance, l'unique exemple d'une génération directe d'un voltage aussi élevé aux bornes des génératrices.

ligne de transport commune anx usines de champ et d'Avignonet. - Elle est établie sur des poteaux entièrement métalliques ayant $1_{2} \mathrm{~m}_{20}$ de hauteur au-dessus du sol. Ce sont des pylones solidement ancrés dans le sol, portant à leur partie supérieure un châssis rectangulaire de $3^{\mathrm{m}} 4 \mathrm{O}$ de large sur $3^{\mathrm{m}} 7^{\circ}$ de hauteur et qui comprennent cinq traverses horizontales recevant chacune quatre isolateurs, lesquels sont placés symétriquement deux par deux de chaque côté de l'axe vertical du pylone. La ligne pourra donc se composer de 20 fils en deux réseaux de ro, de part et d'autre de l'axe des pylones, chaque usine ayant l'usage d'un réseau. Ces deux réseaux sont séparés par un intervalle de $\mathrm{I}^{\mathrm{m}} 50$ et protégés l'un de l'autre, sur chaque pylone, par un écran en toile métallique permettant d'effectuer les réparations sur l'un d'eux sans interrompre le service du voisin. La hauteur au-dessus du sol de la traverse la plus basse est de $8 \mathrm{~m} 50$. La distance de deux poteaux consécutifs est de 60 mètres.

Actuellement, la ligne ne comporte en tout que 15 conducteurs, dont 6 pour Champ et 9 pour Avignonet. Chaque usine emploie un type d'isolateur différent, ayant résisté à l'épreuve d'une tension de 62000 volts pendant 30 minutes. Les poteaux sont reliés électriquement entre eux et mis à la terre, tous les kilomètres, par un fil spécial aboutissant à de larges plaques de cuivre placées dans le sol aux endroits les plus conducteurs. Cette précaution a pour but d'éviter tout danger pouvant résulter de contacts entre les fils et les poteaux à la suite de bris d'isolateurs.

Cette ligne est divisée en plusieurs tronçons aux extrémités desquels sont installés des postes de couplage, comprenant des parafoudres à résistances liquides et des interrupteurs-commutateurs de fils, et à proximitẻ desquels sont des maisons de garde avec poste téléphonique. On pourra ainsi, en cas d'avarie à un groupe de fils, sur un tronçon quelconque de l'une ou l'autre usine, lui substituer un autre groupe sans interruption de service et mettre à la terre les conducteurs d'un côté de la ligne pour permettre les réparations. Une ligne téléphonique, installée sur des supports distincts, relie les usines génératrices aux postes de couplage.

Aux endroits où la ligne traverse le Drac, elle est supportée par des pylones doubles ; aux passages sur le chemin de fer les pylones sont reliés par des passerelles métalliques.

Comme on le voit, aucune précaution n'a été épargnée pour assurer la sécurité du fonctionnement de cette installation grandiose, menée à bonne fin au milieu de diffcultés peu communes. Le mérite de son succès désormais certain revient à la $\mathrm{Ciu}$ Franco-Suisse pour l'Industrie Electrique et à M. Boissonnas, son distingué directeur.

L'énergie produite par l'usine génératrice de Champ est distribuée, dans un rayon de 60 kilónètres, aux nombreux industriels échelonnés le long des vallées de la Fure et de la Morge. Actuellement,
57 usines utilisent 3300 chevaux électriques sur les 4300 que la Société tient à la disposition des abonnés aux bornes de leurs tableaux de distribution. L'exploitation régulière a commencé le ier avril dernier et, depuis cette époque, la distribution de force fonctionne à l'entière satisfaction des papetiers, fabricants de soieries. taillandiers et mdustriels qui utilisent cette énergie.

L'énergie de l'usine d'Avignonet est distribuée en des points très élorgnés les uns des autres. Ainsi la Cie des Mines de la Mure, malgré qu'elle ait le combustible sur place, reconnaissant à l'exemple de nombreuses mines en France et à l'étranger, l'avantage de l'emploi de l'électricité, n'a pas hésité à transformer son matériel et à devenir le client de la Societé Grenobloise de Force et Lamière. Le tramway récemment concédé de Grenoble à Villard-de-Lans, doit emprunter sa force motrice à la mème source. Enfin, une importante fraction de ce transport de torce doit prochainement atreindre la région de Vienne et celle de Bourgoin, distante de roo kilomètres de l'usine génératrice. Souhaitons que tant d'efforts soient couronnès de tout le succès qu'ils méritent.

Société des Forces Motrices du Haut-Grésivandanl. - L'usine de Chapareillan comprend deux turbines Neyret-Brenier et deux turbines Bouvier, de Grenoble, tournant toutes les quatre à 360 tours. Les deux premières actionnent des alternateurs Labour produisant du courant monophasé (1500 ampères sous 160 volts) porté ensuite à 5000 volts par quatre transformateurs Labour; les deux autres turbines sont accouplées à des alternateurs du Creusot donnant directement du monophasé à 5000 volts. Cette énergie sert à l'éclairage des communes de Barraux et de Chapareillan.

L'usine de Pontcharra-sur-Bréda, alimentée par une chute de 42 mètres, comporte six unités génératrices de 6 oo HP chacune, dont trois sont actuellement en service. Elles sont composées :

$1^{\circ}$ De turbines centripètes à aspiration, système NeyrctBrenier, à axe horizontal, tournant à 300 tours, munies d'un régulateur automatique de vitesse.

$2^{n}$ D'alternateurs triphasés qui leur sont accouplés par des joints Raffart et produisant chacun 2400 ampères sous 120 volts; deux sont du type CErlikon, l'autre d'Alioth. Ces machines sont indépendantes, mais peuvent s'accoupler électriquement. A chacune d'elles correspondent deux transformateurs Aliơh élevant la tension à 1000 volts. Ces transformateurs sont à l'étage au-dessus de la salle des machines; ils reçoivent le courant par des barres omnibus et le rendent sur les câbles de départ à la naissance de la ligne de transport.

L'équipement de cette usine est remarquable par la simplicité des dispositifs adoptés, la sécurité de manœuvre des appareils et la robustesse du matériel. Elle sert à l'éclairage de Chambéry et d'un grand nombre de communes de la région tant dans l'Isère que dans la Savoie.

Installations de Traction électrique. - Usines de Lancey, - M. Bergès fait servir la houllle blanche à toutes les applications et autant de divisions ferions-nous dans notre classification, autant de fois devrions-nous mentionner les usines de Lancey. A la fabrication du papier il a adjoint :

I0 L'éclairage, dans un rayon de 15 kilomètres, de dix communes de la vallée du Grésivaudan au moyen d'une station dans laquelle quatre alternateurs monophasés produisent du courant à 10000 volts; 
$2^{\circ}$ La production de l'énergie mécunique actionnant l'usine du tramway électrique de Grenoble à Chapareillan.

Ici $M$. Bergès ne fournit, en effet, que l'eau nécessaire à la marche de cette dernière usine. Un compteur à l'entrée de l'usine mesure le débit de l'eau absorbée sous la pression constante ( 500 mètres) de la colonne d'alimentation, laquelle actionne trois unités génératrices de 375 chevaux. Celles-ci sont composées de turbines Brénier-Neyret à axe horizontal et libre déviation, tournant à 325 tours, accouplées par des joints Raffart à des génératrices compound du Creusot fournissant du courant continu à la tension de 600 volts. Montées en série, ces génératrices alimentent une distribution à trois fils, laquelle est formée par deux conducteurs de contact et les rails. Les voitures automotrices possèdent deux moteurs de 35 HP auxquels correspondent respectivement deux trolleys s'appuyant chacun sur un câble; la différence de potenticl entre ces deux câbles est de 1200 volts et de 600 volts seulement entre la terre à l'état neutre et chaque conducteur aérien( $I$ ). Chaque automotrice peut recevoir une charge utile de 5 tonnes et franchir des rampes de $40 \mathrm{~m} / \mathrm{m}$ par mètre, en remorquant deux voitures chargées de ro tonnes. La longueur du réseau est de 43 kilomètres. C'est le premier exemple, en France, d'une Compagnie de tramway empruntant sa puissance mécanique à des tiers, et il est à désirer qu'il se généralise.

\section{Chemin de fer électrique P.-L.-NI. du Fayet à Chamonix.}

Usines génératrices de Servoz et des Chavants. - L'installation de ces deux usines est à peu près identique. Toutes deux, en effut, comportent 4 génératrices à courant continu de $200 \mathrm{Kw}$, dont une de réserve, accouplées chacune, par un manchon élastique, à une turbine à axe horizontal de 325 fl. Dans l'usine de Servoz, les turbines sont centripètes et tournent à la vitesse de 450 tours par ninute, sous une hauteur de chute de 40 mètres. Dans l'usine des Chavants, les turbines sont centrifuges, à libre déviation, hydropneumatisées, à admission partielle et à débit constant. La hauteur de chute est de 94 mètres. Dans les deux usines, les dynamos sont excitées séparément par deux dynamos de $40 \mathrm{Kw} .$. dont une de réserve, qu'entrainent deux turbines centrifuges de $60 \mathrm{HP}$. Celles-ci sont comme les grosses turbines de l'usine des Chavants, à aspiration, à libre déviation, hydropneumatisées, à admission partielle, mais à débit variable par un distributeur commandé à la main ou par un régulateur automatique de vitesse.

Enfin, chaque usine est pourvue d'un pont roulant de dix tonnes.

Voie et matériel roulant. - Cette ligne qui, sur son parcours de 19 kilomètres, doir racheter une différence de niveau de près de 500 mètres (l'altitude du Fayet est de 580 mètres et celle de Chamonix de 1037 ), présente deux fortes rampes, l'une de omog sur 2155 mètres, l'autre de omo\& sur 1386 mètres. Les autres déclivités ne dépassent pas omoz. Les trains, composés en général de quatre voitures de 32 places et d'un fourgon à bagages où se trouve à l'avant le compartiment du mécanicien,gravissent ces rampes par adhérence. A cet effet,toutes les voitures sont automotrices et possèdent chacune deux moteurs excités en série, dont la puissance individuelle à la jante des roues varie de 55 HP à 32 HP pour des vitesses de ro à 20 kilomètres à l'heure. Un servo-moteur à air comprimé, inventé par M. Auvert, permet au mécanicien de commander les appareils de mancuvre électrique de toutes les voitures du train. Les moteurs sont couplés toujours en parallèle, car le patinage, dans les fortes rampes, est bien moins dangereux que si les moteurs étaient mis en série. Au démarrage, on supprime successivement les trois résistances placées en série avec les moteurs, puis, pour augmenter la vitesse, on shunce les inducteurs.

(1) Voir la conférence de M. Perst sur la traction électrique.
En outre des freins ordinaires à air comprimé, toutes les voitures sont pourvues d'un frein à mâchoires qui vient serrer latéralement un rail placé au centre de la voie sur toute la longueur des deux rampes. Ces freins peuvent être manouvrés à main ou par l'air comprimé.

Le courant est recueilli par des frotteurs sur des rails Vignole placis latéralement à la voie. Ces rails, pesant 34 kilog. 400 par mètre courant, reposent sur des cales en bois paraffiné servant d'isolateur. Dans les gares, ils sont protégés par une enveloppe en bois et aux passages à niveau, on les a remplacés par un câble souterrain en cuivre.

Les rails de la roie de roulement, dont l'écartement est de un mètre, servent de conducteur de retour.

Les dépenses totales de l'installation, y comprises les deux usines électriques, se sont élevées à environ 10.500 .000 francs.

\section{Stations électriques pour traction de la Société Hydro-Electrique de Vizille}

La Société des voies ferrées du Dauphiné voulant transformer ses lignes à vapeur de Grenoble à Domène et de Grenoble à Vizille, en lignes électriques, s'est adressée à la Sociétú hydro-electrique de Vizille pour la fourniture du courant et l'installation des stations et du réseau de distribution. Cette Société avait construit, à 4 kil. de Vizille, une usine alimentée par les eaux des lacs Laffrey sous une chute te $360 \mathrm{~m}$. et comprenant deux groupes électrogènes de qoo HP pour donner directement du courant triphasé à 10000 volts, destiné à l'éclairage et à la force motrice. On établit alors un nouveau groupe de 50 kilo-watts produisant du courant continu sous 600 volts pour alimenter le tronçon de la ligne située près de Vizille.

L'autre partie de lik ligne reçoit son courant d'une sous-station placée à La Galochère, entre Grenoble et Uriage. Là, le courant triphasé à 10000 volts, fourni par l'usine de Vizille, est transformé en continu à 600 volss par deux groupes de trois transformateurs monophasés montés en rriangle et deux commutatrices de $\mathrm{r} 50$ kilo-watts. Ces commutatrices, remarquables surtout par leur fonctionnement avec du courant alternatif à la fréquence de 50 périodes, tournent à 750 tours par minute et ont, par suite, 8 pôles. Elle peuvent supporter des surcharges momentanées allant jusquà $100 \mathrm{o} / \mathrm{o}$ ct ont un pouvoir de synchronisation très considérable. Toutes ces diverses machines ont élé fournies par la Compagnie Westinghouse.

Afin d'éviter les á-coups brusques sur les alternateurs de l'usine de Viz lle qui servent également pour l'éclairage, on ainstallé, dans la sous-station, une batterie tampon formée de 287 éléments Tudor et possédant une capacité de 252 ampères-heure. Un survolteur, composé d'un moteur triphasé asynchrone et d'une génératrice à courant continu de 15 kilo-watts, permet la charge de la batterie et son réglage auromatique pendant la marche normale. La batterie est, en outre, utilisée pour le démarrage des commutatrices qui fonctionnent alors comme des moteurs à courant continu.

Usines d'Electrochimie. - Société Électrochimique de la Romanche. - La destination première de l'usine de Livet était la fabrication du carbure de calcium et l'électrométallurgie, aussı est-elle équipée en vue de ces opérations.

Elle possède actuellement cinq groupes électrogènes d'une puissance individuelle de I 250 chevaux et deux petites unités de ${ }^{7} 5 \mathrm{HP}$, pour l'excitation des génératrices et l'éclairage des bâtiments. Toutes les turbines fournies par la maison Neyret-Brénier sont à axe horizontal. Les moteurs de $1250 \mathrm{HP}$, du type centripète, à débit constant et aspiration, tournent à 350 tours ; ceux de $175 \mathrm{HP}$, du type Girard, à libre déviation et débit variable avec tiroir sur le distributeur, tournent à 500 tours. Tous sont munis de régulateurs automatiques à servo-moteur hydraulique, système NeyretBrenier.

Les génératrices sont des alternateurs Thury, à induit fixe et inducteur tournant, à 16 pôles, donnant du courant monophasé ( 70 volts; 46,6 périodes; $\cos . p=0,80$ ). Les 
excitatrices, également du système Thury, sont à double collecteur et donnent du courant à 70 volts.

Cette usine dispose donc actuellement d'une puissance de 6000 chevaux qui peut être facilement, et à peu de frais, doublée, gràce aux dispositions prévues dans son aménagement. Ainsi, sur ie collecteur des turbines, on a ménagé des prises permettant d'alimenter de nouveaux groupes électrogènes qui seront placés dans une salle de machines parallèle à la salle actuelle, et dont l'emplacement a été réservé dès le début des constructions.

Les bâtiments industriels destinés à l'électrochimie ne comportent pas moins de $4000 \mathrm{~m}^{2}$ de superficie couverte. Ils sont desservis par des voies Decauville, et disposés de manière que les diverses opérations s'effectuent méthodiquement, suivant un cycle continu et sans fausses mancuvres. Signalons que l'évacuation des poussières et des gaz des fours a lieu par le canal de fuite de l'usine, au noyen d'une batterie de pulvérisation.

On sait qu'à la suite d'un récent marché (I), c'est l'usine de Livet qui doit faire l'éclairage électrique de Grenoble. Lorsque, à cet effet, la transformation partielle de son équipement électrique sera opérée, il lui restera encore une grosse pa"t d'énergie disponible pour l'électrométallurgie.

Usine des Clavanx. - Propriété de la Compagnie Universelle d'Acétylène, elle a été, en rgoo-19or, prise en location par la Socicté delectrochimie, dont le siège-social est à Paris, 2, rue Blanche.

Voici, sommairement, quel est son outillage: Les turbines à axe horizontal, système Neyret-Brenier, sont du type centripète à aspiration, d'une puissance de 540 chevaux ; la vitesse normale est de 250 tours par minute. La charge étant sensiblement constante, il n'y a pas de régulateurs automatiques; un grand papillon, mû à la main, placé sur la dérivation individuelle de chaque turbine, sert à gouverner ces moteurs.

Il y a cinq groupes électrogènes, de 375 kilo-watts chacun; les dynamos sont manchonnées directement sur l'arbre des turbines. Un petit groupe de 80 kilo-watts alimente l'éclairage et divers électro-moteurs de 5 à 30 chevaux répartis dans l'usine.

Les dynamos de 375 kilo-watts sont à courant continu, multipolaires, à deux collecteurs indépendants dont chacun fournit 2500 amperes sous $7^{b}$ volts.

II y a :

$$
\begin{aligned}
& 2 \text { machines Thury-Creusot à } 12 \text { pöles. } \\
& 2 \text { machines Alioth } \\
& 1 \text { machine Creusot } S \quad \text { à } 16 \text { pôles. }
\end{aligned}
$$

La crise qui atteint momentanément toutes les branches de l'industrie électrochimique, fait que cette usine n'utilise que trois machines. La fabrication, purement électrolytique, comprend la production du sodium et du peroxyde de sodium, par les procédés Castner et Hulin, ainsi que celle de l'oxylithe, par les procédés $G .-F$. Jaubert, procédés dont la Société d'Electrochimie est concessionnaire exclusive pour la France.

(1) Voir, à ce sujet, l'article très détaillé de M. P. Bovgault : L'alimentation des Villes en eau et énergie électrique, $2^{\text {ute }}$ partie, paru dans le $n^{\circ} 4$ de La Houille Blanche.
Usine de "La Volta". - Elle comporte neuf groupes électrogènes de 500 chevaux formés de turbines Neyret-Brenier, sans régulateurs, et accouplées par des joints élastiques à des génératrices dont six, type du Creusot, sont à courant continu, et trois sont des alternateurs Alioth. L'équipement de la salle des machines est remarquable a tous les points de vue : disposition méthodique des groupes électrogènes, des connexions, des appareils de surveillance et de commande; matériel remarquablement entretenu; espaces et clarté ménagés à profusion etc.

L'usine électrochimique proprement dite, comprend actuellement 19 bâtiments couvrant une superficie totale d'environ $7500 \mathrm{~m}^{2}$. Elle exploite les procédés OutheninChalandre pour l'électrolyse des chlorures alcalins.

Quand on visite cette puissante et belle installation, on est frappé de l'unité de conception qui a présidé à l'édification de son ensemble. Depuis la prise d'eau jusqu'aux magasins recevant les produits fabriqués, on voit que tout a étê prévu sur un plan unique, dont l'exécution ne laisse rien à désirer. Chaque ouvrage, chaque appareil concourt de la façon la plus rationnelle au but que l'on s'était proposé d'atteindre.

Les honneurs de la visite ont été faits aux membres du Congrès de la manière la plus courtoise par M. Georges Coutagne, ancien éléve de l'Ecole Polytechnique, docteur ès-sciences, administrateur délégué de la Société.

Usine de La Praz - Elle comporte plusieurs séries de groupes électrogènes. La conduite à haute pression de 72 mètres alimente : $1^{0}$ seize groupes de 500 chevaux formés chacun d'une turbine Neyret-Brénier genre Girard, avec aspiration de 9 mètres, à axe horizontal, et d'une dynamo Thury (Creusot), de 3000 amperes et 130 volts, accouplées par des manchons élastiques; $2^{\circ}$ trois dynamos de 250 HP sous 50 volts; une dyramo de 250 HP sous 120 volts, alimentant des électro-moteurs et des lampes à incandescence; une dynamo de 120 chevaux sous 65 volts, pour l'éclairage par lampes à arcs; deux alternateurs, l'un de $800 \mathrm{HP}$, servant aux essais, et l'autre de 350 HP; 30 deux turbines, l'une de 350 HP conduisant des laminoirs et l'autre de 250 HP. Soit au total 10500 chevaux engendrés par cette partie de l'installation.

La conduite à basse pression (35 mètres), utiliscée l'été lors des débits abondants alimente: $1^{\circ}$ deux groupes de 800 chevaux à courant continu, fournissant du courant à 65 volts, actionnés par des turbines à axe vertical de la maison Escher-Wyss; $2^{0}$ deux dynamos unipolaires de 350 HP sous 40 volts; une dynamo de 50 HP sous 65 volts servant à l'éclairage; $3^{\circ}$ une petite turbine commandant des transmissions mécaniques. Cette deuxième partie de l'installation fournit ainsi environ 2400 chevaux.

L'usine dispose donc d'une puissance totale de 12500 che. vaux en eaux moyennes.

Tous les groupes électrogènes sont installés sur une seule ligne. Les turbines n'ont pas de régulateurs; l'admission se fait au moyen d'un robinet vanne et d'un papillon.

Le courant est employé à la fabrication de l'aluminium, des ferro-métalliques (ferro-chrome et autres) pour alliages de dilution, et de l'acier. M. Héroult, le distingué directeur 
de cette usine, a très aimablement fait assister les membres du Congrès à une coulée d'acier au four électrique.

Usine de Chedde. - Elle possède 12 groupes turbinedynamo, non compris les moteurs accessoires. Les turbines sont du système Girard à axe horizontal et à libre déviation; elles tournent à 230 tours et ont été construites par MM. Brenier et Neyret, de Grenoble.

Les dynamos ont été tournies : quatre par CErljkon; quatre par le Creusot et quatre par la Sociéte Alsacienne; elles sont à 8 pôles, à courant continu et chacune de $560 \mathrm{Kw}$.

Les conditions particulièrement favorables de l'installation générale de Chedde, permettent d'y obtenir le chevalan à $13 \mathrm{fr}$. sur l'arbre des turbines et à $22 \mathrm{fr}$. aux bornes des dynamos; c'est, dit M. de la Brosse, dans l'ouvrage que nous avons déjà cité, le type des installations de grandes forces à bas prix.

Les usines de Chedde, exploitées par MM. Corbin et $\mathrm{Cie}^{\mathrm{i}}$, utilisent la chute de 140 metres pour la fabrication des produits chimiques par l'électrolyse, notamment des chlorates et perchlorates alcalins.

Les bâtiments couvrent une surface de I 300 mètres carrés; le principal a 130 mètres de longueur, 60 mètres de large et $21^{\text {m}} 50$ de hauteur; les salles des turbines et des dynamos ont chacune 120 mètres de long.

Usine de Prémont. - Située sur l'Arve, en aval de La Praz, elle appartient à la Sociélé d'Electrochimie. Elle utilise une chute de 75 mètres avec un débit de $6^{\mathrm{m}^{3}}$ environ. Son équipement comprend : quatre turbines Bouvier de $450 \mathrm{HP}$ et douze turbines Neyret-Brenier de 250 HP commandant chacune des dynamos Thury. Cette usine est affectée a l'électrométallurgie de l'aluminium et à diverses fabrications électro-chimiques.

Usines de Rionpéroux. - Bien connues en ce qui concerne la fabrication du papier, nous devons, en terminant, signaler leur installation hydro-électrique comportant trois turbines Neyret-Brenier à axe horizontal, de 850 chevaux et une de $250 \mathrm{HP}$, fonctionnant sous 34 mètres de chute, accouplées à des dynamos de divers systèmes, dont le courant sert à la fabrication de produits chimiques.

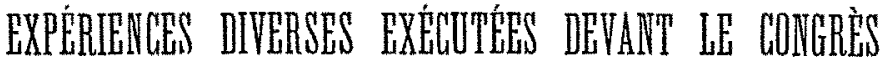

\section{ESSAIS D'ISOLATEURS A 70000 VOLTS}

La Société Grenobloise de Force et Lumiere avait invité les membres du Congrès à se rendre à son laboratoire pour leur montrer comment se comportent les divers types d'isolateurs actuellement en usage sur les lignes de trans. port de force à haute tension. Ces expériences reproduisaient les dispositils adoptés pour l'essai des isolateurs que cette Société devait employer dans la construction de la ligne de 35 kilomètres, entre Champ et Moirans, à 26000 volts, dont il a été question plus haut dans la description des installations visitées par le Congrès.

Les différents modèles d'isolateurs mis en expérience étaient placés dans deux bacs métalliques isolés du sol. Dans le premier bac, on avait placé deux rangées d'isolateurs, la tête en bas, plongeant dans une couche d'eau de quelques centimètres d'épaisseur. L'eau et la tête des isolateurs étaient en communication avec la borne haute tension d'un transformatearr-élévateur ( 1 io: 70000 volts) dont lautre borne était reliée à deux barres de cuivre placées au-dessus du bac ct desquelles descendaient de petites chainettes plongeant dans l'intérieur des isolateurs. L.a tension de 70000 volts pouvait ainsi être obtenue entre les parois extérieures et intérieures des isolateurs. Lune des rangées était formée d'isolateurs secs, l'autre d'isolateurs mouillés. Cette disposition permettait de se rendre compte de l'influence de la vapeur d'eau atmosphérique dans le cas des transports de force à très haute tension.

Dés que la tension atteignait environ roooo volts, les isolateurs mouillés étaient entourés d'effluves, puis d'aigrettes et de chenilles lumineuses qui persistaient jusqu'à ce que la plus grande partie de l'eau recouvrant les isolateurs ait disparu. Les isolateurs secs ont supporté très facilement la tension de 70000 volts; un amorçage d'arc avait parfois lieu entre l'eau et la chainette de communication, en produisant un bruit sec, même assourdissant. A partir de 40000 volts, les chaînettes conductrices étaient lumineuses et on pouvait facilement les suivre dans l'obscurité.

Sur le second bac, on avait placé une traverse en bois sur laquelle étaient fixés par leurs tiges les différents modèles d'isolateurs expérimentés dans le premier bac. Au-dessus de ce système, on avait disposé une sorte de trémie d'oú tombait de l'eau en gouttelettes très divisées, ce qui imitait assez bien la pluie. Dans cette expérience, les deux bornes haute tension du transformateur-élévateur étaient reliées, l'une à la tête des isolateurs, l'autre à leurs tiges. Ces isolateurs étaient donc ainsi placés dans les conditions où ils se trouveraient sur un conducteur présentant entre la terre et lui une différence de potentiel de Toooo volts.

Les mèmes phénomènes que ceux observés dans la première expérience ont été constatés, mais avec une intensité remarquablement plus accentuée; le soufre et le chanvre qui avaient servi à sceller les isolateurs sur leurs tiges, ont pris feu.

Dans ces expériences, la tension primaire ( $\mathrm{I}$ Iov.) du transformateur était réglée de o à I lo volts, à l'aide de self appropriées disposées sur un tableau spécial. Le couran d'alimentation de l'appareil venait d'un poste de transformateurs que la S.G.F.L. a installé à côté de son labora. toire. Elle recevait, dans un transformateur de MM. Schneider et $\mathrm{C}^{\mathrm{ic}}$, du courant à 15000 volts, l'abaissait à 2000 volts, puis l'envoyait dans un transformateur de la maison Grammont pour l'abaisser une seconde fois à la tension de I Io volts nécessaire au fonctionnement du transformateurélévateur Brown-Boveri, servant aux essais.

\section{EXPERIENGES SUR L'ALUMINOTHERMIE}

L'aluminothermie est un procédé de réduction et de chauffage des métaux sans intervention de carbone que M. Goldschmidt a rendu pratique pour certaines applications, telles que le brasage et la soudure autogène.

On sait que l'aluminium, s'il ne brûle pas à l'air, par contre s'oxyde avec un énorme dégagement de chaleur en réduisant les oxydes des autres métaux (à part les métaux alcalins et alcalino-terreux). Par exemple, le mélange d'un oxyde de chrôme avec de la poudre d'aluminium, donne, la réaction étant amorcée par un jet de flamme, du chrôme métallique avec un dégagement de chaleur tel que la masse du mélange est rapidement amenée à l'état liquide. M. Goldschmidt évalue à plus de $3000^{\circ}$ la température à laquelle a lieu cette réaction. 
La condition essentielle pour que l'opération réussisse bien est de réaliser un mélange aussi intime que possible de l'aluminium et de l'oxyde métallique à réduire. L'amorçage de la réaction s'obtient en employant un mélange dans la proportion de ogr. 5 de poudre impalpable d'aluminium et de 2 gr. 5 de bioxyde de baryum finement pulvérisé, dont on projette quelques grammes à la surface du mélange à traiter que l'on a placé dans un creuset. La chaleur dégagée par cette poudre d'allumage à laquelle on met le feu au moyen d'une allumette, s'étend très rapidement à toute la masse contenue dans le creuset et l'on obtient presque instantanément du métal fondu.

$M$. Gall après avoir rappelé ce phénomène aux membres du Congrès qu'il avait réunis à cet effet dans l'une des annexes de l'usine de Prémont, leur a montré une très intéressante application de l'aluminothermie.

Au-dessus d'une sorte de moule en tôle garni de terre réfractaire, renfermant, fortement pressées l'une contre l'autre, les deux extrémités de rails que l'on voulait souder, était disposé un creúset contenant un mélange de poudre d'aluminium et d'oxyde de fer; à la surface on a projeté de la poudre d'allumage et quelques secondes après que l'on y eût mis le feu avec une allumette, le creuset était rempli de fer liquide à une température extrêmement élevée; on a alors fait couler le métal liquide dans le moule. On a pu constater que la chaleur dégagée avait fondu les bouts des rails qui s'étaient parfaitement soudés. Il a coulé du creuset d'abord du corindon liquide qui s'est solidifié au contact du métal froid et que l'on a pu enlever facilement avec le métal réducteur lorsqu'on eût retiré le moule.

L'aluminothermie peut donc être considérée comme une application indirecte de l'énergie électrique - puisque l'aluminium est un produit du four électrique - d'un empioi sans doute couteux, mais du moins commode en certains cas.

\section{EXPÉRIENGES AVEC LA "CHEDDITE"}

Après avoir fait visiter la chute de l'usine de Chedde et la salle des machines aux membres du Congrès, MM. Corbin et Cie leur ont montré une application intéressante des chlorates que l'on fabrique dans cette usine; ce sont des explosifs chloratés appelés "Cheddites".

Ils sont très sûrs et très puissants ainsi que le démontrent les diverses expériences suivantes exécutées sous les yeux du Congrès:

Le choc dine balle tirée sur un paquet de cartouches de dynamite le fait exploser; le même essai, fait sur un, paquet de cartouches de Cheddite, ne détermine pas d'explosion; pour bien démontrer que la Cheddite explose, on fait détonner le reste du paquet avec une amorce.

Cette expérience pronve que cet explosif résiste même aux chocs les plus violents.

On verse de l'acide sulfurique concentré sur une caisse de Cheddite elle s'entlamme, mais il n'y a pas d'explosion; c'est une simple combustion que l'on éteint avec un peu d'eau; on fait exploser le reste au moyen d'une amorce. Ceci démontre que la Cheddite est très sûre en cas d'incendre.

On détermine ensuite la rupture d'un rail de chemin de fer avec deux cartouches de Cheddite et un simple bourrage au sable; puis la rupture d'un poteau de bois avec quelques cartouches, sans aucun bourrage; enfin, on fait exploser des torpilles de Cheddite scus leau; toutes expériences attestant que ce nouvel explosif est très puissant et très brisant.

\section{VOYAGE EN SUISSE}

Le dimanche matin, if septembre, ceux des Congressistes qui avaienr résolu d'aller voir dans le Valais, l'utilisation de la houille blanche et que la pluie de la veille n'avait pas découragés, se mirent en route pour les Montets, la Forclaz, Martigny et Brig. Il furent bientôt récompensés de leur persévérance par un gai soleil qui, chassant les nuages, leur offrit dans toute sa splendeur le spectacle des beautés grandioses de ce pays enchanteur.

Cette excursion dans la vallée du Haut-Rhône offrait, au point de vue technique, trois attractions: les travaux du Simplon; le transport de force du Bois-Noir à Lausanne par le systeme Thury et l'aménagement des forces motrices du lac Tanay à Vouvry. Nous nous bornons à en exposer de simples vues d'ensemble desquelles se dégage un enseignement pratique. Le lecteur saura où trouver le détail de ces installations déjà si bien décrites en de nombreuses publications illustrées de planches et de photographies.

Les Travaux du Simplon. - Comme on le sait, le tunnel du Simplon sera le plus long de tous les ouvrages de ce genre; il doit avoir 19.730 m. Attaqué par ses deux extrémités à Brig et à Iselle, les deux galeries vont à la rencontre l'une de l'autre et, au total, elles ont actuellement une longueur de 10 kilomètres environ. Les travaux doivent être terminés en mai 1904 .

Ce tunnel, au lieu d'être comme les autres formé d'une seule voûte, comprendra deux galeries parallèles sur toute la longueur, chacune formant un tunnel à une voie, l'un pour la voie montante et l'autre pour la voie descendante. Actuellement on ne perce qu'un seul de ces tunnels sur une section de $5 \mathrm{~m}$. go en hauteur et 5 mètres en largeur; la deuxième galerie n'est percée que sur une section de $3 \times<2,50$; elle fait office de galerie de service servant à l'évacuation des déblais, des eaux et à la ventilation. Ces deux galeries sont reliées entre elles tous les 200 mètres par des galeries transversales et que l'on bouche ensuite au fur et à mesure de l'avancement. Seul, un premier tunnel sera donc d'abord ouvert à la circulation.

Les Congressistes ont visité les chantiers de Brig situés à l'altitude de 680 mètres. Ils sont immenses; à eux seuls leurs bâtiments occupent une superficie couverte de Ir.370 $\mathrm{m}^{2}$. L'eau sous pression y est le principal moteur, presque seule elle fait face à tous les travaux mécaniques. Une canalisation en ciment armé, amène l'eau du Rhône, capté a Mürel, à quatre kilomètres environ en amont de Brig, dans un réservoir à 50 mètres au-dessus des chantiers. Ce réservoir alimente des turbines dont l'ensemble constitue une puissance motrice de 1500 chevaux. Les unes de ces turbines actionnent des compresseurs d'air, des ventilateurs, des machines frigorifiques et des pompes de refoulement d'eau pour les perforatrices. Les autres actionnent les dynamos servant à l'éclairage des cours, des bâtiments et à la transmission du mouvement à certaines machines telles que grues électriques, ponts roulants, etc...

Les travaux occupent 1800 ouvriers italiens dont 1200 travaillent dans les galeries. L'outil primordial de l'ceuvre sont les perforatrices rotatives à fleurets d'acier durci qui 
fonctionnent sous une charge d'eau de 80 kilos, attaquant la roche qui est de gneiss. L'avancement dans chaque galerie est conduit par des équipes d'élite de 50 ouvriers, commandées par deux contre-maîtres sous la direction d'un ingénieur. Les autres équipes, plus nombreuses et composées de manœuvres, ne sont occupées qu'au déblaiement.

Les matériaux explosés sont soumis au marinage, c'est-àdire arrosés pour faciliter le déblaiement. Ce sont des locomotives à air comprimé qui transportent ces matériaux hors des galeries. La ventilation s'opère par les méthodes génćralement adoptées dans tous les travaux de ce genre. Mais signalons que l'éclairage des galeries se fait par l'acétylène.

L'avancement moyen est de 5 mètres par 24 heures et la longueur de la galerie de Brig est très approximativement de 5 kilomètres à l'heure actuelle.

Ainsi l'on voit, dans ces travaux, la puissance mécanique de l'eau mise au service des applications les plus diverses: génération de l'énergie électrique servant aux besoins les plus variés, compression d'air pour transmission de mouvement et ventilation, alimentation et mise en marche de machines outils, etc... Sans la merveilleuse souplesse de son emploi, le tunnel du Simplon ne serait pas à l'état d'avancement remarquable où l'on peut le voir aujourd'hui.

On est frappé de l'ordre qui a présidé à l'exécution matérielle des plans conçus par les ingénieurs. Suivant la saisissante comparaison de l'un de nos collaborateurs, les dispositions adoptées pour le percement du tunnel réalisent presque le schéma de l'attaque d'une place de guerre. La place à laquelle il faut faire brèche est ici le Monte-Léone; les galeries sont les tranchées, les perforatrices, l'artillerie, et le chantier avec ses dynamiteries, ses voies ferrées, ses usines à compression d'air, à refoulement d'eau, etc... représenteavec beaucoup d'analogie un grand parc de siège. La similitude se poursuit même jusque dans l'installation des douches et du vestiaire, des bureaux et des logements des ingénieurs, de l'infirmerie et de l'hôpital... Mais, à l'inverse des engins de guerre mobilisés pour la lutte fratricide, ce puissant outillage fonctionne pour rapprocher des peuples!

Dans les autres installations, l'emploi de l'eau n'est pas aussi varié, mais alors, là, c'est à l'Electricité que revient le rôle de fractionner, distribuer, répandre l'énergie. Ce n'est pas à dire cependant qu'il n'y ait rien d'intéressant à apprendre dans les applications remarquables que les ingénieurs suisses ont su faire de leurs forces hydrauliques.

L'Usine du Bois-Noir. - Le choix du coude du Bois-Noir pour l'installation d'un barrage mobile et la solution élégante qui a été adoptée pour sa construction, sont dignes d'attention. Il s'agissait de retenir l'eau du Rhône de façon que le niveau en restât toujours constant et que l'ícoulement, pendant les hautes eaux, ne fut pas entravé par les travaux de captage. La solution a été des plus simples et des plus heureuses.

Une sorte de pont, jeté sur le Rhône, est divisé en trois travées, deux petites accotées aux rives et une grande, de quarante-huit mètres de portée au-dessus du thalweg. Des deux petites travées, l'une domine l'entrée du canal de prise et l'autre laisse passer le trop-plein de l'eau du fleuve, quand toutes les vannes qui s'appuient sur la travée du milieu sont abaissées. On peut, à volonté et selon les besoins, abaisser tout ou partie de ces vannes de la travée du milieu et ne relever le plan d'écoulement du Heuve que de la hauteur strictement indispensable pour ramener le niveau à la cote utile.

Les ouvrages pour la décantation qui font suite à ceux de la prise d'eau, sont traités avec simplicité et solidité et remplisserst parfaitement leur objet.

L'eau est amenée sur les turbines par un tuyau en fer forgé de $2^{\mathrm{m}} 70$ de diamètre intérieur, long de $47^{\circ}$ mitres et dont l'épaisseur croît de 7 à ro millimètres. Deux autres tuyaux semblables sont prévus.

L'usine comporte huit turbines Francis à axe horizontal accouplées directement et fonctionnant avec une aspiration de 6 mètres.

Cinq ont une puissance de $1000 \mathrm{HP}$ à 300 tours avec un débit de 3100 litres et une chute de 32 à 34 mètres.

Deux autres turbines font chacune i 20 chevaux à 750 tours avec un débit de 380 litres; enfin mentionnons une petite turbine à aubes.

Nous estimons inutle de rappeler ici la description de l'équipement électro-mécanique si remarquable de cette station dont tous les périodiques ont déja donné des descriptions détaillées. Bien que de telles installations se puissent rencontrer ailleurs, celle-ci supporte tous les parallèles.

Usine d'Evionnaz. - Elle appartient à la commune de Lausanne. La puissance de l'eau du Rhône y est, sauf la part nécessaire à l'éclairage de St-Maurice et des environs qui se fait en triphasé, convertie en courant continu et transportée par le système série, souis 20000 volts, sur une distance de 56 kilomètres à l'usine réceptrice à Lausanne.

Usine de la Pierre du Plan. - Ici, l'énergie est utilisée de deux manières. Une partie du courant abaissée à 600 volts, sert à la traction des tramways de Lausanne. L'autre partie, transformée en courant triphasé à 3000 volts, fournit la lumière dans la banlieue de Lausanne et dans h ville après abaissement à 125 et 216 volts.

Cette usine est remarquablement installée, tout y est équipé avec un soin méticuleux de façon que jamais le consommateur d'énergie n'ait à craindre, même une minute, une interruption de service. Une machine à vapeur de secours est là pour parer à toutes éventualités.

Un égal souci de la sécurité a pésidé à l'installation des canalisations électriques : les connexions sont dans des logements séparés, clairs et commodément accessibles au personnel de service; les appareils sont couplés de manière à réduire la main-d'œuvre à un minimum extrềme et à faciliter on ne peut plus la surveillance; partout, beaucoup de clarté et des soins exemplaires d'entretien et de propreté.

Cette usine semble bien devoir exprimer la formule des installations à venir.

Les Forces motrices du lac Tanay. - Capier un lac situé à plus de goo mètres d'altitude au-dessus du point oú sa puissance doit être utilisée est assurément une cuvre dont la hardiesse étonne et fait réfléchir plus 
d'un ingénieur. C'est à ce problème que s'est attaqué $M$. Boucher et il l'a résolu avec une simplicité et un bonheur parfaits. Nous donnons, dans la note ci-dessous, résumant la description qu'il a publiée dans le Bullelin technique de la Suisse ronande, les détails les plus frappants de cette installation unique. ( 1 )

C'est grâce aux progrès de l'art du tubiste que cette magistrale conception a pu être réalisée.

Usine de Vouvry. - Elle fournit l'énergie sous forme de courant monophasé à 6000 volts et à 50 périodes et peut alors se mettre en parallèle avec l'usine de la Grande-Eau, située à lo kilomètres de là, en amont d'Aigle. Ces deux usines se prêtent ainsi un mutuel secours et quand le débit de l'eau baisse à celle de la Grande-Eau, celle du Lac Tanay lui transmet une aide proportionnelle.

Il semble qu'il y ait là d'utiles exemples dont pourront s'inspirer les ingénieurs dans des cas analogues.

Autres installations visitées par le Congrès. - Les congressistes ont terminé leurs promenades par la visite des usines bien connues de la Coulouvrenière et de Chêvres. Près de Chêvres, sur la rive droite du Rhône, les congressistes ont pu voir une usine d'électrochimie qui emprunte son énergie à l'usine voisine. Cette usine d'électrochimie est fermée. Son histoire nous a été contée de deux manières différentes et, ce qui semble en résulter, c'est que l'exploitant a dû cesser pour diverses causes dont l'une, et non des moindres, aurait été l'irrégularité avec laquelle l'usine de Chêvres lui donnait l'énergie.

Cette dernière, usine communale, satisfaisant d'abord aux besoins communaux, ne donnait à son client que son superflu, quand elle en avait.

\section{(1) La plus haute chute d'eau du monde}

La Société des Forces motrices de la Grande-Eau utilise à Vouvry (Suisse) une chute effective de 920 mètres pour la distribution de la force motrice et de l'éclairage, par l'électricité. Cette chute alimentée par le lac Tanay qui reçoit annuellement plus de 12000000 de $\mathrm{m}^{\mathrm{m}^{3}}$ d'eau, peut donner par an sur larbre des turbines 30000000 HP heure.

L'installation prévue pour $10000 \mathrm{HP}$ et pouvant en donner actuellement 2000 est destinée à renforcer l'usine hydro-électrique construite par la même Société à 10 kilomètres de Vouvry. Les alternateurs de ces deux usines étant de même voltage et de même fréquence sont couplés en parallèle sur le même réseau.

La prise d'eau est constituée par un puits de 30 mètres de profondeur où debouche une crevasse naturelle communiquant avec le lac et donnant aux basses eaux 200 litres par seconde. L'eau est d'abord amenée par 2 tunels, puis par un tuyau en tôle de 1200 mètres de long et de o m. 80 de diamètre, jusqu'à l'endroit oủ commence la grande descente. En ce point, où la pression ne dépasse pas 2) mètres, on a placé une soupape de retenue destinée à arrêter leau en cas de rupture de la canalisation à l'aval. Une colonne montante de 25 métres de haut atténue le coup de bélier dû à la fermeture de cette soupape. La grande descente de la canalisation, qui commence alors et s'étend sur 1040 métres en ligne à peu près droite, comprend 2 sections. La première, longue de 635 mètres est formée d'un tuyau unique de $500 \mathrm{~m} / \mathrm{m}$ de diamètre extérieur avec une épaisseur de métal croissant de $7 \dot{a} \times 1,5 \mathrm{~m} / \mathrm{m}$. Dans la seconde section, longue de 1300 metres, la conduite principale se subdivise en deux tuyaux de $341^{\mathrm{m} / \mathrm{m}}$ de diamètre extérieur. L'épaisseur du méral croit de 8 à $18 \mathrm{~m} / \mathrm{m}$. Ces conduites sont en acier extra-doux Martin Siemens sans rivure.Les joints à emboîtements sont serrés par des boulons, ce qui a rendu inutile tout amarrage au sol de la canalistion et la pose de massifs de maçonnerie près des coudes, Du reste, la canalisation est recou-
Il y a là une leçon de choses capable de faire réfléchir les industriels - s'il y en a - qui seraient partisans de la municipalisalion des services de production de l'énergie et aussi ceux qui, trop confiants, louent cette énergie sans vérifier, au préalable, si le producteur sera toujours à mème de faire face à ses engagements.

En plus de ces installations. les membres du Congrès ont encore visité le sanatorium de L.eysin et l'organisation du petit chemin de fer à crémaillère Abt qui y tait accéder.

Au sanatorium de Leysin, on saisit sur le vif ce que l'on peut appeler l'industrialisalion de l'art de guérir. Sont-ce donc, en effet, autre chose que des usines à guérison que ces palais si plaisants et si confortables où tout est combiné, soins perfectionnés, site admirable, pour faire oublier ses douleurs au malade!

Le $\mathrm{I} 7$ septembre, les derniers congressistes se séparaient à Genève.

\section{LE MOIS HYDR0-ÉLEGTRIQUE en France et à l'Etranger}

\section{INFORMATIONS DIVERSES}

\section{La Houille Blanche en Limousin}

On est parfois tenté de croire que le Dauphiné et la Savoie sont les seules régions où lon puisse avantageusement utiliser la Houlle blanche. S'ii est vrai que ces contrées sont particulièrement favorisées à ce point de vue, elles n'en ont cependant pas le monopole absolu. Les Pyrénées, l'Auvergne, le Jura et les Vosges possédent de belles

verte d'une épaisse couche de terre destinée à la préserver des variations de température et contre la chute de rochers. Chacun des deux tuyaux de la seconde section est terminé par une vanne tiroir en acier, mobile à la main, destinée à fermer leau en cas de réparation à l'aval. Cette vanne est suivie d'une autre à commande hydraulique se manœuvrant à distance et depuis le tableau de distribution. Celleci doit se fermer rapidement en cas d'accident. Néanmoins, pour éviter un coup de bélier dangereux, cette termeture ne s'eflectue pas en moins de 5 secondes:

L'usine contient actuellement 4 turbines d'une puissance normale de $500 \mathrm{HP}$, possedant individuellement deux distributeurs de $500 \mathrm{HP}$ chacun, mais dont un seul est réglable. Deux de ces turbines sont des roues Pelton en bronze. Les deux autres sont composées d'un disque massif en fonte à la périphérie duquel, à droite et à gauche, se trouvent des ailettes courbes. Chaque distributeur est alors ciouble, donnant un jet pour les aubages de droite et un autre pour ceux de gauche. Ces turbines sont munies de remarquables régulateurs hydrauliques à servo-moteur, dont le fonctionnement est parfait. Elles entrainent chacune à la vitesse de 1000 tours par minute, un alternateur monophasé a 6000 volts; les turbines proprement dites étant montées en porte-à-faux sur les bouts des arbres de ces alternateurs. L'excitation de ceux-ci se fait, soit par des excitatrices montées en bout d'arbre, soit par deux petites dynamos actionnées chacune à 2000 tours par minute, à l'aide d'une turbine de 25 Hp possédant un seul distributeur réglable à main. Une petite batterie d'accumulateurs sert en outre de secours pour l'excitation.

Pour les 2000 premiers thevaux établis, l'installation a coùté environ 800000 francs. On estime a 300000 francs ta dépense nécessaire pour doubler cette puissance, Cette usine ne fonctionna que depuis le mois d'avril 1902. 